\title{
MARKOV EXTENSIONS, ZETA FUNCTIONS, AND FREDHOLM THEORY FOR PIECEWISE INVERTIBLE DYNAMICAL SYSTEMS
}

\author{
G. KELLER
}

\begin{abstract}
Transfer operators and zeta functions of piecewise monotonic and of more general piecewise invertible dynamical systems are studied. To this end we construct Markov extensions of given systems, develop a kind of Fredholm theory for them, and carry the results back to the original systems. This yields e.g. bounds on the number of ergodic maximal measures or equilibrium states.
\end{abstract}

\section{INTRODUCTION}

\section{A. Background.}

Piecewise invertible dynamical systems have attracted considerable attention during the last two decades. They occur in the literature under various names, the most prominent are perhaps: piecewise monotonic transformations [Lasota/Yorke, 1973], $f$-expansions or number-theoretical algorithms, see [Watermann, 1970] or [Schweiger, 1973] for references, and one-sided subshifts, in particular subshifts of finite type or topological Markov chains, cf. [Denker et al., 1976]. They all can be described as triples $(X, T, \mathscr{Z})$ where $X$ is a topological space, $\mathscr{Z}$ is a finite or countable partition of $X$, and $T: X \rightarrow X$ is a mapping whose restrictions $T_{Z}$ to elements of $\mathscr{Z}$ are injective.

[Hofbauer, 1986] investigates topological properties of (a restricted class of) such systems. He constructs a topological Markov chain on a countable state space in such a way that the system under consideration is a continuous factor of this chain (in fact, it is a one-block factor). Although the factor map is generally not finite-to-one, it is nice enough to carry over a great part of the chain's topological structure to the underlying system yielding in this way a description of the set of nonwandering points and of the measures of maximal entropy. Such results ignore, however, a possible smooth structure of the space $X$ and a priori ideas about what subsets of $X$ are small in a measure-theoretic sense.

To be definite, consider the family of systems $\left([0,1), T_{p}, \mathscr{Z}_{p}\right), 0<p<1$, where $\mathscr{Z}_{p}=\{[0, p),[p, 1)\}, T_{p}(x)=p^{-1} x(0 \leq x<p), T_{p}(x)=(1-p)^{-1}(x-p)$ $(p \leq x<1)$. All $T_{p}$ leave the Lebesgue measure on $[0,1)$ invariant and are

Received by the editors March 9, 1987. 28D05.

1980 Mathematics Subject Classification (1985 Revision). Primary 58F20, 47B10; Secondary 
measurably isomorphic to a one-sided Bernoulli-shift with stationary probability vector $(p, 1-p)$. Their metric entropy $p \log p+(1-p) \log (1-p)$ is maximized for $p=\frac{1}{2}$ such that the Lebesgue measure is singular to the measure of maximal entropy if $p \neq \frac{1}{2}$. Nevertheless we are mostly interested in properties of orbits $\left(T_{p}^{n} x\right)_{n=1,2, \ldots}$ valid for Lebesgue-almost all $x \in[0,1)$.

This motivates the attempt to investigate extensions $(\hat{X}, \hat{T}, \hat{\mathcal{Z}})$ of "smooth" systems which at the same time inherit the "smooth" local structure of the system and show a global Markov structure similar to that exploited by Hofbauer. As a consequence of the Markov structure, "smooth" probability densities on $\hat{X}$ are transformed by the action of $\hat{T}$ into "smooth" densities, provided that $\hat{T}$ itself is "smooth" enough. This allows a systematic study of the spectral properties of the Perron-Frobenius operator of $\hat{T}$ acting on spaces of "smooth" functions on $\hat{X}$. To number-theorists this operator is better known as Kuzmin operator, and in statistical mechanics generalized versions of it are called transfer operators. (I shall adopt this last name.) In a second step these results must be interpreted for the system $(X, T, \mathscr{Z})$.

During my work on these problems I learned that the same techniques can be used for exploring equilibrium states on various subshifts. For subshifts of finite type [Mayer, 1980] has done interesting work in this direction (in the context of one-dimensional spin systems with long-range interactions). From his work I learned how to use the theory of nuclear operators for investigating dynamical systems. The results presented here apply to a much broader class of subshifts including sofic systems (see [Coven/Paul, 1975], [Weiss, 1973]) and many coded systems (see [Blanchard/Hansel, 1986]).

\section{B. Organization and results.}

$\S 2$ begins with a quick review of the spectral theory for quasicompact operators and of Fredholm-determinants for nuclear operators on Banach-spaces. Both concepts are combined in the definition of quasinuclear operators (Definition 2.3. Some technical aspects of this definition are motivated by later applications.) The main result for quasinuclear operators $P$ (Theorem 2.5) describes that part of their spectrum that can be characterized by the zeroes of an analytic function associated with $P$ and called the Fredholm-determinant of $($ Id $-z P)$.

The next three sections are independent of these functional analytic preparations, and the reader might prefer to begin his reading of this paper with definitions, basic facts, and simple examples for piecewise invertible dynamical systems and with the construction of Markov extensions for given systems ( $\S 3$ ), followed by an introduction to transfer operators and (Ruelle-)zeta functions for weighted piecewise invertible systems and their Markov extensions $(\S 4)^{1}$ and

\footnotetext{
1 Very briefly: If $T: X \rightarrow X$ is an at most countable-to-one transformation and if $g: X \rightarrow \mathbb{C}$ is a "weight function", then $\left(P_{g} f\right)(x)=\sum_{y \in T^{-1} x} f(y) g(y)$ is the associated transfer operator and $\zeta_{g}(z)=\exp \left(\sum_{n=1}^{\infty} \frac{z^{n}}{n} \sum_{T^{n} x=x} g(x) g(T x) \cdot \ldots \cdot g\left(T^{n-1} x\right)\right)$ is the corresponding zeta function.
} 
some considerations on analytic structures for such weighted systems $(\S 5)$ including notions of (generalized) holomorphic functions and expanding systems. $\S 6$ is the core of this paper. We investigate transfer operators $P_{g}$ acting on a space $\mathscr{H}^{\infty}$ of bounded holomorphic functions: Theorem 6.1 gives conditions under which transfer operators of Markov extensions of expanding systems are quasicompact on $\mathscr{H}^{\infty}$ and describes in some detail the isolated eigenvalues of $P_{g}$ and their associated eigenspaces. Our main result, Theorem 6.2, gives additional conditions under which $P_{g}$ is quasinuclear and under which (some of) the isolated eigenvalues of $P_{g}$ are just the inverses of the poles of $\zeta_{g}(z)$. This uses heavily Theorem 2.5. Finally, Proposition 6.10 and Theorem 6.11 relate the peripheral spectrum of $P_{g}$ to invariant measures on irreducible components of the Markov extension.

While the zeta function of a weighted piecewise invertible system and that of its Markov extension are often equivalent in the sense that they have the same poles and zeroes in the domain referred to by Theorem 6.2, the relations between the spectral properties of the corresponding transfer operators are more delicate: Proposition 7.1 provides some general criteria for an eigenvalue of the original system to be also an eigenvalue of the extended system. Unfortunately I was not able to obtain a useful, general result in the other direction, except for peripheral eigenvalues (Lemma 7.3). ${ }^{2}$ Nevertheless the results from $\S \S 6$ and 7 show that for many transfer operators $P_{g}$ associated to a weighted piecewise invertible system there is $r<r\left(P_{g}\right)$ (= spectral radius of $P_{g}$ ) such that the inverse of each eigenvalue $\lambda$ of $P_{g}$ with $|\lambda|>r$ is a pole of the corresponding zeta function with multiplicity equal to the algebraic multiplicity of $\lambda$.

In $\S 8$ some of the previous results are translated into the "language" of equilibrium states. For example, if the greatest positive eigenvalue of the transfer operator $\hat{P}_{g}$ of the Markov extension of a weighted system is simple, then there is a unique equilibrium state $\mu$ for $\log g$ (Theorem 8.3.b), and if $(T, \mu)$ is weakly mixing, then the partition $\mathscr{Z}$ is weak Bernoulli under $T$ with an exponential mixing rate (Theorem 8.5 ). (Both theorems say a bit more than indicated here.)

In $\S 9$ we touch upon several applications of Theorem 6.1: It is pointed out, how the spectral theory for $P_{g}$ can be used to approach various probabilistic limit theorems via the routes (a) $L^{2}$ - approximation, (b) strongly mixing stationary processes and functionals of them, (c) Fourier- or Laplace-transforms. We also relate exponential extinction rates to the pressure of $T$ restricted to a certain "repelling" subsystem (Proposition 9.7) and give a rigorous proof of the fact that long transients behave stochastically just like typical trajectories from the "repelling" subsystem.

I have chosen three classes of examples in order to illustrate the sometimes rather general definitions and results:

\footnotetext{
${ }^{2}$ For a rather special case the reverse implication has been proved in [Hofbauer/Keller, 1984].
} 
(I) Topological Markov shifts, sofic systems ${ }^{3}$

These are among the simplest piecewise invertible systems, and they are included only, because everything reduces to ideas well-known to most readers.

(II) Up-and-down-counters ${ }^{4}$

This is a class of subshifts which are neither of finite type nor sofic (i.e. homomorphic images of subshifts of finite type). Using a rather explicit formula for their zeta function $\zeta_{1}(z)$ we show e.g. that they have unique maximal measures (8.7).

(III) Piecewise analytic and expanding interval transformations ${ }^{5}$

These are maps $\tilde{T}:[0,1] \rightarrow[0,1]$ for which there is a partition $0=a_{0}<$ $a_{1}<\cdots<a_{N}=1$ such that each $\tilde{T}_{\mid\left(a_{t-1}, a_{l}\right)}$ is monotone and extends holomorphically to a map $\tilde{T}_{i}$ defined on a complex neighbourhood of $\left[a_{i-1}, a_{i}\right]$ and satisfying $\left|\tilde{T}_{i}^{\prime}\right| \geq \alpha>1$. If $\phi:[0,1] \rightarrow \mathbb{C}$ is such that each $\phi_{\mid\left(a_{i-1}, a_{i}\right)}$ extends holomorphically to the same neighbourhood as the corresponding $\tilde{T}_{i}$ and if $g=\exp \phi$,

$$
g_{\infty}=\lim _{n \rightarrow \infty}\left(\sup _{x}\left|g(x) \cdot g(T x) \cdot \ldots \cdot g\left(T^{n-1} x\right)\right|\right)^{1 / n}
$$

then $\zeta_{g}(z)$ extends to a meromorphic function on $\left\{|z|<g_{\infty}^{-1}\right\}$, and $\lambda^{-1}$ is a pole of $\zeta_{g}$ if and only if $\lambda$ is an eigenvalue of $\hat{P}_{g}$, the transfer operator of the Markov extension of $\tilde{T}$. In this case, the multiplicities of $\lambda^{-1}$ as a pole and of $\lambda$ as an eigenvalue coincide. If $g=1 /\left|\tilde{T}^{\prime}\right|$, i.e. if $P_{g}$ is the classical Perron-Frobenius operator, then each eigenvalue $\lambda$ of $P_{g}$ acting on $B V[0,1]^{6}$ that satisfies $|\lambda|>g_{\infty}^{1 / 2}$ is the inverse of a pole of $\zeta_{g}$. The multiplicity of $\lambda$ as an eigenvalue is less than or equal to the multiplicity of $\lambda^{-1}$ as a pole (see (7.7)). In this case it is also true that all equilibrium states for $\log g$ are absolutely continuous with respect to Lebesgue measure, and the number of ergodic equilibrium states equals the multiplicity of the eigenvalue 1 of $P_{g}$ acting on $B V[0,1]$ (cf. 7.7). This number, in turn, coincides with the multiplicity of the pole at 1 of $\zeta_{g}(z)$ (cf. 6.14 and 7.7). An informal discussion of the Fredholm theory for transfer operators of interval maps can be found in [Oono/Takahashi, 1980].

\section{Discussion.}

I feel that the rather heavy machinery used for the description of the general setting and the proof of the main result needs some additional justification:

(1) Analyticity results for Fredholm determinants and for the inverses of zeta functions have been obtained so far only for very simple dynamics (topological Markov shifts over a finite alphabet) but quite general $g$, see e.g. [Ruelle,

\footnotetext{
${ }^{3}$ See $3.12,4.8,5.9,6.12,7.5$.

${ }^{4}$ See $3.13,4.9,5.9,6.13,7.6,8.7$.

5 See $3.15,4.10,5.10,6.14,7.7,8.8$.

6 The space of functions of bounded variation on $[0,1]$.
} 
1976] or [Pollicott, 1986], and, on the other hand, for more general dynamics (piecewise monotonic interval maps) but rather simple $g$ (piecewise constant), see e.g. [Takahashi, 1981], [Hofbauer/Keller, 1984], or [Mori, 1985]. The combination of more complex dynamics and more general $g$ necessitates a theorem like 2.5 and justifies the use of holomorphic functions in 6.2. It seems possible, however, that a clever combination of Pollicott's arguments with Theorem 2.5 could yield similar results for $P_{g}$ acting on spaces of Lipschitz-continuous functions or on spaces of functions of bounded variation.

(2) The general framework introduced in $\S \S 3$ and 4 allows to treat a class of systems much broader than just interval transformations (which have a very special Markov extension). This is illustrated by the subshifts in Example II.

(3) Even for interval transformations, and even if one is not interested in zeta functions, the use of Markov extensions and of holomorphic functions has its merits: It seems quite difficult to obtain rigorous results about extinction rates and transient behaviour as presented in $\S 9 . C$ by studying transfer operators acting on spaces of functions of bounded variation, cf. [Keller, 1984].

(4) All results apply to higher-dimensional systems, too, but it seems to be nontrivial to verify the assumptions of Theorems 6.1 and 6.2 for fairly general classes of such systems. The complex $\beta$-transformation that maps the unit square (regarded as a subset of the complex plane) to itself by $z \mapsto \beta z \bmod \mathbb{Z}^{2}$ $(|\beta|>1)$ should be a good candidate to test ones ideas for more general twodimensional piecewise analytic and expanding maps. It already turned out to be tractable from a related point of view in [Keller, 1979].

\section{QUASICOMPACT AND QUASINUCLEAR OPERATORS}

\section{A. Quasicompact operators.}

Throughout this section $P$ is a linear operator on some complex Banachspace $(B,\|\|$.$) . Generalizing Definition V-3-1 and Lemma V-3-1 in [Neveu,$ 1964], we say that $P$ is quasicompact if there is $k \in \mathbb{N}$ and a compact operator $K: B \rightarrow B$ such that $\left\|P^{k}-K\right\|<r(P)^{k}$ where $r(P)$ is the spectral radius of $P$. The existence of the limit

$$
r_{K}=\lim _{n \rightarrow \infty}\left(\inf \left\{\left\|P^{n}-K\right\|: K \text { a compact operator on } B\right\}\right)^{1 / n}
$$

is trivial. Obviously $r_{K}<r(P)$, and it follows from Lemma VIII.8.2 in [Dunford/Schwartz, 1958] that every spectral point $\lambda$ of $P$ with $|\lambda|>r_{K}$ is isolated in the spectrum $\sigma(P)$ of $P$ and that the corresponding eigenprojection $E_{\lambda}$ has a finite-dimensional range. Thus we obtain the following restricted spectral representation for a quasicompact operator $P$ (cf. [Kato, 1976], III.6.5):

For any $r>r_{K}$ there are finitely many spectral values $\lambda_{1}, \ldots, \lambda_{N}$ with $\left|\lambda_{i}\right|>r$. All of them are eigenvalues, and $P$ can be decomposed as

$$
P=\sum_{i=1}^{N} \lambda_{i}\left(E_{i}+N_{i}\right)+Q
$$


where $E_{i}$ is the finite-dimensional eigenprojection corresponding to $\lambda_{i}, N_{i}=$ $\left(P-\lambda_{i}\right.$ Id $) E_{i}$ its eigennilpotent ${ }^{7}$, and $Q$ is a linear operator with $r(Q) \leq r$. Furthermore $E_{i} E_{j}=0$ (for $\left.i \neq j\right), E_{i} N_{i}=N_{i} E_{i}=E_{i}$ (for all $i$ ), and $E_{i} Q=Q E_{i}=0$ (for all $\left.i\right)$.

In particular, all $\lambda_{i}$ are poles of the resolvent of $P$, and $\lambda_{i}$ is a pole of order $\nu_{i}$ if and only if $\left(P-\lambda_{i} \text { Id }\right)^{\nu_{i}} E_{i}=0$ but $\left(P-\lambda_{i} \mathrm{Id}\right)^{\nu_{i}-1} E_{i} \neq 0$ (Theorem VII.3.18 in [Dunford/Schwartz, 1958]).

With this notation we obtain a representation for $P^{n}$ :

$$
P^{n}=\sum_{i=1}^{N} \lambda_{i}^{n} E_{i}\left(\sum_{k=0}^{\nu_{i}-1}\left(\begin{array}{l}
n \\
k
\end{array}\right) N_{i}^{k}\right)+Q^{n} .
$$

For $T: B \rightarrow B$ let $\operatorname{ker}(T)=\{x \in B: T x=0\}$ and $\operatorname{range}(T)=\{T x: x \in$ $B\}$. Theorem VII.3.24 in [Dunford/Schwartz, 1958] implies

$$
\begin{gathered}
\operatorname{ker}\left(P-\lambda_{i} \operatorname{Id}\right)^{\nu_{i}}=\operatorname{range}\left(E_{i}\right) \simeq B / \operatorname{range}\left(\mathrm{Id}-E_{i}\right), \\
\operatorname{range}\left(P-\lambda_{i} \mathrm{Id}\right)^{\nu_{i}}=\operatorname{range}\left(\mathrm{Id}-E_{i}\right)
\end{gathered}
$$

for all $\lambda_{i}$. In particular $\left(P-\lambda_{i}\right.$ Id $)$ is a Fredholm operator.

\section{B. The essential spectrum.}

The above spectral decomposition motivates the following definition: For a bounded linear operator $P$ on $B$ the nonessential spectrum is the set of all isolated spectral points $\lambda$ of $P$ for which $\operatorname{range}(P-\lambda \mathrm{Id})$ is closed and $\bigcup_{n \geq 0} \operatorname{ker}\left((P-\lambda \text { Id })^{n}\right)$ is finite-dimensional. Its complement in $\sigma(P)$ is just the essential spectrum ess $(P)$ of $P$ in the sense of [Browder, 1961, Definition 11]. The essential spectral radius is defined as

$$
r_{\text {ess }}=\sup \{|\lambda|: \lambda \in \operatorname{ess}(P)\}
$$

From the results listed in part $\mathrm{A}$ it is obvious that for quasicompact operators $r_{\text {ess }} \leq r_{K}$, and, using "measures of noncompactness", [Nussbaum, 1970] proved that for arbitrary bounded linear operators

$$
r_{\text {ess }}=r_{K}
$$

holds.

\footnotetext{
${ }^{7}$ Id denotes the identity mapping on $B$.
} 


\section{Nuclear operators.}

Denote by $B^{\prime}$ the space of bounded linear functionals on $B$. According to [Grothendieck, 1956, Chapter II.1], a linear operator $P$ on $B$ is nuclear (or of trace class), if it can be represented as

$$
P=\sum_{i=1}^{\infty} \alpha_{i} y_{i}^{\prime} \otimes x_{i}
$$

where $x_{i} \in B, y_{i}^{\prime} \in B^{\prime},\left\|x_{i}\right\|,\left\|y_{i}^{\prime}\right\| \leq 1$, and $\sum_{i=1}^{\infty}\left|\alpha_{i}\right|<\infty . y_{i}^{\prime} \otimes x_{i}$ represents the operator on $B$ mapping $x$ to $y_{i}^{\prime}(x) \cdot x_{i}$. The trace norm $\|P\|_{T}$ is the infimum of all $\sum_{i=1}^{\infty}\left|\alpha_{i}\right|$, the $\alpha_{i}$ arising from a representation (2.6) of $P$.

Observe that rigorously the right-hand side of $(2.6)$ is an element of the normcompletion $B^{\prime} \hat{\otimes} B$ of $B^{\prime} \otimes B$ (ibid.), and in general the same operator $P$ can be represented by different elements from $B^{\prime} \hat{\otimes} B$. However, if $P$ is $p$-summing for some $0<p \leq 2 / 3$, i.e. if $P$ has a representation (2.6) with $\sum_{i=1}^{\infty}\left|\alpha_{i}\right|^{p}<\infty$ for some $0<p \leq 2 / 3$, then there is only one element in $B^{\prime} \hat{\otimes} B$ representing $P{ }^{8}$ Since we are only working with $\frac{2}{3}$-summing operators, we call $P$ nuclear, if it is nuclear in the sense of Grothendieck and has a unique representation in $B^{\prime} \hat{\otimes} B$. This allows us to define the trace ${ }^{9}$ of a nuclear $P$ unambiguously as

$$
\operatorname{tr}(P)=\sum_{i=1}^{\infty} \alpha_{i} \cdot y_{i}^{\prime}\left(x_{i}\right)
$$

where $\alpha_{i}, x_{i}, y_{i}^{\prime}$ are taken from any representation (2.6), and the Fredholm determinant ${ }^{10}$

$$
\operatorname{det}(\operatorname{Id}-z P)=\exp \left(-\sum_{n=1}^{\infty} \frac{z^{n}}{n} \operatorname{tr}\left(P^{n}\right)\right)
$$

for $|z|<1 / \varlimsup_{n \rightarrow \infty}\left(\operatorname{tr}\left(P^{n}\right)\right)^{1 / n}$. Theorem 4 and its corollaries in [Grothendieck, 1955] tell us that $\operatorname{det}(\operatorname{Id}-z P)$ is an entire function and

$$
\operatorname{det}(\operatorname{Id}-z P)=\prod_{i}\left(1-z \lambda_{i}\right)
$$

where the product extends over all eigenvalues $\lambda_{i}$ of $P$ counted according to their multiplicities. The sequence $\left(\lambda_{i}\right)_{i=1}^{\infty}$ is $r$-summable if $P$ is $p$-summing and $r^{-1}=p^{-1}-\frac{1}{2}$. Finally (ibid.)

$$
\operatorname{tr}(P)=\sum_{i} \lambda_{i}
$$

\section{Quasinuclear operators.}

Remember that the defining property of a quasicompact operator $P$ is its approximability (in the sense of part A) by compact operators. The resulting

\footnotetext{
${ }^{8}$ See Corollary 3 of Theorem 4 in Chapter II, $\S 1$ of [Grothendieck, 1955].

${ }^{9}$ See Chapter I, §3, no. 2 of [Grothendieck, 1955] or Chapter II, Proposition 1 of [Grothendieck, 1956].

${ }^{10}$ See Chapter II.3 of [Grothendieck, 1956].
} 
decomposition (2.2) asserts a much nicer approximation: For any $r>r_{\text {ess }}$ there is a finite-rank (and a fortiori nuclear) operator $F$ such that spectral radius $(P-F)<r$ and $(P-F) F=F(P-F)=0$. So there is nothing special, from this point of view, about approximability by nuclear operators. However, the operator $F$ is not explicitly known in general, and the Fredholm determinant of $F$, which might serve as an approximate Fredholm determinant for $P$, cannot be described in known terms. Therefore we shall define quasinuclear operators in terms of "effective" approximation schemes of nuclear operators.

2.1. Definition. A family $\mathscr{F}$ of continuous projections on a Banach space $B$ is a projection net if

(a) $\alpha, \beta \in \mathscr{F}$ implies $\alpha \beta=\beta \alpha \in \mathscr{F}$ and there is $\gamma \in \mathscr{F}$ with $\alpha \gamma=\alpha$, $\beta \gamma=\beta$.

(b) $\|\alpha\| \leq C$ for some $C>0$ and all $\alpha \in \mathscr{F}$.

The projection net $\mathscr{F}$ is continuous if

(c) $\lim _{\alpha \in \mathscr{F}} \alpha(x)=x$ for all $x \in B$ in the norm topology of $B .{ }^{11}$ If $\mathscr{F}_{0}=\mathscr{F} \cup\{$ Id $\}$, then $\mathscr{F}_{0}$ is also a projection net.

Given a linear operator $P$ and a projection net $\mathscr{F}$ we fix some further notation: For $\alpha \in \mathscr{F}$ denote by $r_{\alpha}$ the spectral radius of

$$
P_{\alpha}:=(\mathrm{Id}-\alpha) P(\mathrm{Id}-\alpha)
$$

and let $r_{\mathrm{as}}=\underline{\lim }_{\alpha \in \mathscr{F}} r_{\alpha}$. Observe that $r_{0}=r(P)$.

2.2. Proposition. Let $P$ be a linear operator on $B$ and let $\mathscr{F}$ be a projection net. If $P \alpha$ is compact ${ }^{12}$ for all $\alpha \in \mathscr{F}$ and if $r_{\mathrm{as}}<r(P)$, then $P$ is quasicompact and $r_{\text {ess }} \leq r_{\text {as }}$.

If additionally $\mathscr{F}$ is continuous and if there is $A>0$ such that for all $m \in \mathbb{N}$ and $0<\varepsilon<r_{\text {as }}$ there is $n \geq m$ with

$$
\varlimsup_{\beta \in \mathscr{F}}\left\|(\mathrm{Id}-\beta) P^{n}(\mathrm{Id}-\beta)\right\| \geq A \cdot\left(r_{\text {as }}-\varepsilon\right)^{n},
$$

then $r_{\mathrm{ess}}=r_{\mathrm{as}}$. In particular $r_{\mathrm{as}}$ is independent of the special choice of $\mathscr{F}$ in this case.

Proof. Fix $\alpha \in \mathscr{F}$. By induction on $n$ we show

$$
P^{n}-P P_{\alpha}^{n-1} \text { is compact for all } n \geq 1 \text {. }
$$

For $n=1$ this is trivial. For $(n+1)$ we have

$$
\begin{aligned}
P^{n+1}-P P_{\alpha}^{n} & =P^{n} P \alpha+P^{n} P(\mathrm{Id}-\alpha)-P P_{\alpha}^{n-1} P(\mathrm{Id}-\alpha)+P P_{\alpha}^{n-1} \alpha P(\mathrm{Id}-\alpha) \\
& =P^{n} P \alpha+P P_{\alpha}^{n-1} \alpha P(\mathrm{Id}-\alpha)+\left(P^{n}-P P_{\alpha}^{n-1}\right) P(\mathrm{Id}-\alpha) .
\end{aligned}
$$

The first term in this sum is compact as $P \alpha$ is, the third one by the inductive hypothesis, and the second term is different from 0 only for $n=1$, in which case its compacity follows from that of $P \alpha$.

$11 \lim _{\mathrm{r} \in \mathscr{G}} \alpha(x)=x$ means that for each neighbourhood $U$ of $x$ there is $\beta \in \mathscr{F}$ such that for all $\alpha \geq \beta$ (i.e. $\alpha \beta=\beta$ ) holds: $\alpha(x) \in U$.

${ }^{12}$ A similar proof yields the same result if the $\alpha P$ are compact. 
Now (2.5) and (2.12) imply $r_{\text {ess }} \leq \lim _{n \rightarrow \infty}\left(\|P\| \cdot\left\|P_{\alpha}^{n-1}\right\|\right)^{1 / n}=r_{\alpha}$, and as $\alpha \in \mathscr{F}$ is arbitrary, $r_{\text {ess }} \leq r_{\text {as }}<r(P)$, i.e. $P$ is quasicompact.

For the reverse inequality let $K$ be any compact linear operator on $B$. Then

$$
\lim _{\beta \in \mathscr{F}}\|(\operatorname{Id}-\beta) K(\operatorname{Id}-\beta)\| \leq(1+C) \cdot \lim _{\beta \in \mathscr{F}}\|(\operatorname{Id}-\beta) K\|=0
$$

by continuity of $\mathscr{F}$ and the fact that $\{K x:\|x\| \leq 1\}$ is relatively compact in $B$. Fix $n \in \mathbb{N}$ such that (2.11) holds. Then

$$
\left\|P^{n}-K\right\| \geq(1+C)^{-2}\left\|(\operatorname{Id}-\beta) P^{n}(\operatorname{Id}-\beta)-(\operatorname{Id}-\beta) K(\operatorname{Id}-\beta)\right\|
$$

for all $\beta \in \mathscr{F}$, and in view of (2.11) and (2.13)

$$
\left\|P^{n}-K\right\| \geq(1+C)^{-2} \varlimsup_{\beta \in \mathscr{F}}\left\|(\operatorname{Id}-\beta) P^{n}(\mathrm{Id}-\beta)\right\| \geq A \cdot(1+C)^{-2} \cdot\left(r_{\text {as }}-\varepsilon\right)^{n} .
$$

Now $r_{\text {ess }} \geq r_{\text {as }}$ follows from Nussbaums's result (2.5) and from (2.1).

2.3. Definition. (a) The linear bounded operator $P: B \rightarrow B$ is $\mathscr{F}$-quasinuclear, if $\mathscr{F}$ is a projection net for which all $P \alpha \quad(\alpha \in \mathscr{F})$ are nuclear and which satisfies:

(i) For $\beta \in \mathscr{F}$ and $n \in \mathbb{N}$ there exists $\lim _{\alpha \in \mathscr{F}} \operatorname{tr}\left(\left(\alpha P_{\beta} \alpha\right)^{n}\right)$. This limit is denoted by $\operatorname{tr}_{\mathscr{F}}\left(P_{\beta}^{n}\right)$, and we set $t_{\beta}=\varlimsup_{n \rightarrow \infty}\left|\operatorname{tr}_{\mathscr{F}}\left(P_{\beta}^{n}\right)\right|^{1 / n}$. For $\beta=0$ we have $P_{0}=P$ and write $t(P)$ instead of $t_{0}$. We also let $t_{\text {as }}:=\underline{\lim }_{\beta \in \mathscr{F}} t_{\beta}$.

(ii) There is $S>0$ such that $\left|\operatorname{tr}\left((\alpha P \alpha)^{n}\right)\right| \leq S^{n} \quad(n \in \mathbb{N}, \alpha \in \mathscr{F})$.

(iii) For each $\varepsilon>0$ there are $C_{\varepsilon}>0$ and $\beta=\beta_{\varepsilon} \in \mathscr{F}$ such that

$$
\left\|\left(\alpha P_{\beta} \alpha\right)^{n}\right\| \leq C_{\varepsilon}\left(r_{\text {as }}+\varepsilon\right)^{n}, \quad\left|\operatorname{tr}\left(\left(\alpha P_{\beta} \alpha\right)^{n}\right)\right| \leq C_{\varepsilon}\left(t_{\text {as }}+\varepsilon\right)^{n}
$$

for $n \in \mathbb{N}$ and $\alpha \in \mathscr{F}_{0}$.

(iv)

$$
\lim _{\alpha \in \mathscr{F}}\left\|\alpha\left(P_{\beta} \alpha\right)^{j} P \beta-P_{\beta}^{j} P \beta\right\|_{T}=0
$$

for all $\beta \in \mathscr{F}$ and $j \in \mathbf{N}$.

(b) Having defined a kind of trace for $\mathscr{F}$-quasinuclear operators in (i) we can extend the definition of a Fredholm determinant (cf. (2.8)) to this class of operators: For $\beta \in \mathscr{F}$ let

$$
\operatorname{det}_{\mathscr{F}}\left(\operatorname{Id}-z P_{\beta}\right)=\exp \left(-\sum_{n=1}^{\infty} \frac{z^{n}}{n} \operatorname{tr}_{\mathscr{F}}\left(P_{\beta}^{n}\right)\right) .
$$

2.4. Remark. (a) The definition of $t_{\beta}$ and $t_{\text {as }}$ depends on the particular choice of $\mathscr{F}$. This should be kept in mind although the above notation ignores this dependence.

(b) Condition (iv) means that $P$ and $P_{\beta}$ map "small" subspaces "close" to ones that are not much "bigger" (in the sense of the filtration $\mathscr{F}$ ). Its special form is motivated by later applications, where it may be satisfied, as in Example 6.14 , not only in the limit but also for "finite" $\alpha$. 
(c) If one does not assume nuclearity of the $P_{\alpha}$ but instead of the $\alpha P$ $(\alpha \in \mathscr{F})$ and if one replaces the inequality in (iv) by

$$
\lim _{\alpha \in \mathscr{I}}\left\|\alpha\left(P_{\beta} \alpha\right)^{j} P \beta-P_{\beta}^{j} P \beta\right\|=0,
$$

then one arrives at a slightly different definition of quasinuclearity that will not be referred to in this paper but might be useful in other situations. The following theorem and its corollary remain valid (see also the footnote to Proposition 2.2).

(d) We did not require $r_{\text {as }}<r(P)$ for $\mathscr{F}$-quasinuclearity. Hence $\mathscr{F}$ quasinuclearity does not imply quasicompactness.

2.5. Theorem. Suppose $P$ is $\mathscr{F}$-quasinuclear. Let $R=\max \left\{r_{\mathrm{as}}, t_{\mathrm{as}}\right\}$. Then

(a) $\operatorname{det}_{\mathscr{F}}(\mathrm{Id}-z P)$ is analytic in $\left\{z \in \mathbb{C}:|z|<R^{-1}\right\}$,

(b) $\operatorname{det}_{\mathscr{F}}\left(\mathrm{Id}-z_{0} P\right)=0$ if and only if $z_{0}^{-1}$ is an eigenvalue of $P$. The multiplicity of $z_{0}$ as a zero of $\operatorname{det}_{\mathscr{F}}(\mathrm{Id}-z P)$ is finite and equals the algebraic multiplicity of $z_{0}^{-1}$ as an eigenvalue of $P$.

2.6. Corollary. Under the assumptions of the theorem the following holds:

(a) All spectral points $\lambda$ of $P$ with $|\lambda|>R$ are eigenvalues of $P$.

(b) $r(P)=t(P)$, if $\operatorname{tr}_{\mathscr{F}}\left(P^{n}\right) \geq 0$ for all $n \in \mathbb{N}$ and if
(1) $t_{\mathrm{as}}<t(P)$ or $t_{\mathrm{as}} \leq r(P)$
and
(2) $r_{\text {as }}<r(P)$ or $r_{\text {as }} \leq t(P)$.

Proof of the corollary. By Proposition 2.2 $P$ is quasicompact and $r_{\text {ess }} \leq r_{\text {as }} \leq R$. This proves (a). If $r(P)>r_{\text {as }}$, we have

$$
r(P)=\max \{|\lambda|: \lambda \text { is an eigenvalue of } P\} .
$$

Hence, if $t(P)>R$ or $r(P)>R$, we obtain from (2.14), Pringsheim's theorem, and Theorem 2.5

$$
t(P)=\varlimsup_{n \rightarrow \infty} \operatorname{tr}_{\mathscr{F}}\left(P^{n}\right)^{1 / n}=\max \left\{|z|^{-1}: \operatorname{det}_{\mathscr{F}}(\operatorname{Id}-z P)=0\right\}=r(P) .
$$

Otherwise we have $r(P) \leq R$ and $t(P) \leq R$, and the additional assumption implies $r(P)=t(P)$.

Proof of the theorem. Fix $\varepsilon>0$ and $\beta=\beta_{\varepsilon} \in \mathscr{F}$ as in Definition 2.3(aiii). For each $\alpha \in \mathscr{F}_{0}\left(\mathrm{Id}-z \alpha P_{\beta} \alpha\right)^{-1}$ exists and is an analytic function of $z$ in $G_{\varepsilon}=\left\{|z|<(R+\varepsilon)^{-1}\right\}$. Let

$$
\begin{aligned}
& A_{\alpha}(z)=-z(\operatorname{Id}-\beta)\left(\operatorname{Id}-z \alpha P_{\beta} \alpha\right)^{-1}(\mathrm{Id}-\beta) \alpha P \alpha \beta, \\
& B_{\alpha}(z)=-z \beta \alpha P \alpha\left(\operatorname{Id}-A_{\alpha}(z)\right) \beta .
\end{aligned}
$$

Then

$$
\begin{aligned}
(\operatorname{Id}-z \alpha P \alpha) & =(\beta+(\operatorname{Id}-z \alpha P \alpha)(\operatorname{Id}-\beta))\left(A_{\alpha}(z)+B_{\alpha}(z)+\operatorname{Id}\right) \\
& =\left(\operatorname{Id}-z \alpha P_{\beta} \alpha-z \beta \alpha P \alpha(\operatorname{Id}-\beta)\right)\left(\operatorname{Id}+B_{\alpha}(z)\right)\left(\operatorname{Id}+A_{\alpha}(z)\right)
\end{aligned}
$$

Although the first identity is not mysterious (its matrix version is Schur's equality, see also Lemma 2 of [Hofbauer/Keller, 1984]), the proof is a bit tricky: 
Let $A=A_{\alpha}(z), B=B_{\alpha}(z)$, and make use of $\alpha \beta=\beta \alpha$. Then

$$
\begin{aligned}
(\beta+ & (\mathrm{Id}-z \alpha P \alpha)(\mathrm{Id}-\beta))(A+B+\mathrm{Id})-(\mathrm{Id}-z \alpha P \alpha) \\
& =B+(\mathrm{Id}-z \alpha P \alpha) A+z \alpha P \alpha \beta \\
& =z(\mathrm{Id}-\beta) \alpha P \alpha \beta+(\operatorname{Id}-z(\mathrm{Id}-\beta) \alpha P \alpha) A \\
& =z(\mathrm{Id}-\beta) \alpha P \alpha \beta+\left(\operatorname{Id}-z \alpha P_{\beta} \alpha\right) A \\
& =z(\mathrm{Id}-\beta) \alpha P \alpha \beta-z(\mathrm{Id}-\beta) \alpha P \alpha \beta+z \beta\left(\mathrm{Id}-z \alpha P_{\beta} \alpha\right)^{-1}(\mathrm{Id}-\beta) \alpha P \alpha \beta \\
& =z \beta(\mathrm{Id}-\beta) \alpha P \alpha \beta+z^{2} \beta \alpha P_{\beta} \alpha\left(\operatorname{Id}-z \alpha P_{\beta} \alpha\right)^{-1}(\operatorname{Id}-\beta) \alpha P \alpha \beta \\
& =0
\end{aligned}
$$

Suppose now that $\alpha \in \mathscr{F}$. As $P$ is $\mathscr{F}$-quasinuclear, all factors occurring in (2.15) are of the type "Id + nuclear operator". Hence

$$
\operatorname{det}(\operatorname{Id}-z \alpha P \alpha)=\operatorname{det}\left(\operatorname{Id}-z \alpha P_{\beta} \alpha\right) \cdot \operatorname{det}\left(\operatorname{Id}+B_{\alpha}(z)\right),
$$

because $\beta \alpha P \alpha(\mathrm{Id}-\beta)$ and $A_{\alpha}(z)$ are "transient" off-diagonal terms which do not contribute to the determinants (see Chapter II.3, Proposition 2 of [Grothendieck, 1956]). We take the $\lim _{\alpha \in \mathscr{F}}$ on both sides of (2.16): As

$$
\operatorname{det}(\operatorname{Id}-z \alpha P \alpha)=\exp \left(-\sum_{n=1}^{\infty} \frac{z^{n}}{n} \operatorname{tr}\left((\alpha P \alpha)^{n}\right)\right) \text { for }|z|<S^{-1}
$$

by (2.8) and Definition 2.3(aii), we have in view of Definition 2.3(ai, ii) and (b)

(2.17) $\lim _{\alpha \in \mathscr{F}} \operatorname{det}(\operatorname{Id}-z \alpha P \alpha)=\operatorname{det}_{\mathscr{F}}(\operatorname{Id}-z P) \quad$ uniformly for $|z|<(S+\varepsilon)^{-1}$

Similarly, but using (iii) instead of (ii) of Definition 2.3(a) one obtains

$$
\begin{aligned}
\lim _{\alpha \in \mathscr{F}} \operatorname{det}\left(\operatorname{Id}-z \alpha P_{\beta} \alpha\right) & =\operatorname{det}_{\mathscr{F}}\left(\operatorname{Id}-z P_{\beta}\right) \\
& =\exp \left(-\sum_{n=1}^{\infty} \frac{z^{n}}{n} \operatorname{tr}_{\mathscr{F}}\left(P_{\beta}^{n}\right)\right) \quad \text { uniformly in } G_{\varepsilon} .
\end{aligned}
$$

As $B_{\alpha}(z)$ is nuclear for all $\alpha \in \mathscr{F}_{0}$ (i.e. also in the limit $\alpha=$ Id), we obtain

$$
\lim _{\alpha \in \mathscr{F}} \operatorname{det}\left(\operatorname{Id}+B_{\alpha}(z)\right)=\operatorname{det}\left(\operatorname{Id}+B_{\mathrm{Id}}(z)\right) \quad\left(z \in G_{\varepsilon}\right)
$$

if we can show that $\lim _{\alpha \in \mathscr{F}} B_{\alpha}(z)=B_{\text {Id }}(z)$ in trace-norm $\|\cdot\|_{T}$, for the determinant is continuous with respect to this norm (see Chapter II.2, Proposition 1 of [Grothendieck, 1956]). To this end fix $l \in \mathbb{N}$, and let $\alpha \geq \beta$ such that for $0 \leq j<l$

$$
\left\|\alpha\left(P_{\beta} \alpha\right)^{j} P \beta-\left(P_{\beta}\right)^{j} P \beta\right\|_{T} \leq\left(r_{\text {as }}+\varepsilon\right)^{j} \cdot l^{-1}
$$


(cf. Definition 2.3(aiv)). ${ }^{13}$ Then (cf. Chapter II, §1, no. 2 of [Grothendieck, 1955])

$$
\begin{aligned}
& \left\|B_{\mathrm{Id}}(z)-B_{\alpha}(z)\right\|_{T}=\left\|z \beta P\left(A_{\mathrm{Id}}(z)-\alpha A_{\alpha}(z)\right)\right\|_{T} \\
& \quad \leq|z|^{2} \cdot\|\beta P\| \sum_{j=0}^{\infty}|z|^{j}\left\|(\mathrm{Id}-\beta)\left(P_{\beta}^{j}-\alpha\left(P_{\beta} \alpha\right)^{j}\right) P \beta\right\|_{T} \\
& \quad \leq|z|^{2} \cdot \mathrm{const} \cdot\left(\frac{1}{l\left(1-|z|\left(r_{\text {as }}+\varepsilon\right)\right)}+\sum_{j=l}^{\infty}\left(|z|\left(r_{\text {as }}+\varepsilon\right)\right)^{j} \cdot\|P \beta\|_{T}\right)
\end{aligned}
$$

by Definition 2.3(aiii), and as $l$ was arbitrary, we obtain

$$
\lim _{\alpha \in \mathscr{F}}\left\|B_{\mathrm{Id}}(z)-B_{\alpha}(z)\right\|_{T}=0 \text { for all } z \in G_{\varepsilon} \text {. }
$$

Now (2.16)-(2.20) imply

$$
\operatorname{det}_{\mathscr{F}}(\operatorname{Id}-z P)=\operatorname{det}_{\mathscr{F}}\left(\operatorname{Id}-z P_{\beta}\right) \cdot \operatorname{det}\left(\operatorname{Id}+B_{\mathrm{Id}}(z)\right) \text { for }|z|<S^{-1},
$$

and the right-hand side of this equation is analytic in $G_{\varepsilon}$. This proves (a) of the theorem.

Let $B(z)=B_{\mathrm{Id}}(z)$, and suppose that $\operatorname{det}\left(\operatorname{Id}+B\left(z_{0}\right)\right)=0$ for some $z_{0} \in G_{\varepsilon}$. The following identity is a generalization of Theorem 5.1 in [Gohberg/Sigal, 1971]:

$$
\begin{aligned}
& \operatorname{tr} \int_{\left|z-z_{0}\right|=r} B^{\prime}(z)(\operatorname{Id}+B(z))^{-1} d z \\
& \quad=\int_{\left|z-z_{0}\right|=r} \frac{d}{d z} \operatorname{det}(\operatorname{Id}+B(z)) \cdot \operatorname{det}(\operatorname{Id}+B(z))^{-1} d z \\
& \quad=2 \pi i \cdot\left(\text { multiplicity of the zero } z_{0} \text { of } \operatorname{det}(\operatorname{Id}+B(z))\right)
\end{aligned}
$$

for small $r$. We prove it: Recall that

$$
B(z)=-z \beta P \beta-z^{2} \beta P(\operatorname{Id}-\beta) P \beta-z^{2} \beta P\left(\sum_{j=1}^{\infty} z^{j} P_{\beta}^{j}\right) P \beta .
$$

Since $\|S P \beta\|_{T} \leq\|S\| \cdot\|P \beta\|_{T}$ for each bounded linear operator $S$ on $B$, $B(z)$ is an analytic function of $z \in G_{\varepsilon}$ with respect to the trace-norm, and $\|B(z)\|_{T}<\frac{1}{2}$ for $|z|<z_{0}$, say. Hence, observing the fact that $\operatorname{tr}(U V)=$ $\operatorname{tr}(V U)$ for nuclear operators $U$ and $V$ on $B$,

$$
\begin{aligned}
& \operatorname{tr}\left(B^{\prime}(z)(\operatorname{Id}+B(z))^{-1}\right)=\operatorname{tr}\left(\frac{d}{d z} \log (\operatorname{Id}+B(z))\right) \\
& \quad=\frac{d}{d z} \operatorname{tr}(\log (\operatorname{Id}+B(z)))=\frac{d}{d z} \log \operatorname{det}(\operatorname{Id}+B(z)) \text { for }|z|<\varepsilon_{0}
\end{aligned}
$$

\footnotetext{
13 This is the only instant of the proof where Definition 2.3(aiv) is needed. If the definition of quasinuclearity is varied as proposed in Remark $2.4(\mathrm{c})$, the proof of the subsequent identity changes only slightly.
} 
i.e.

$$
\operatorname{tr}\left(B^{\prime}(z)(\operatorname{Id}+B(z))^{-1}\right) \cdot \operatorname{det}(\operatorname{Id}+B(z))=\frac{d}{d z} \operatorname{det}(\operatorname{Id}+B(z))
$$

for $|z|<\varepsilon_{0}$, and by analytic continuation (2.23) continues to hold on $G_{\varepsilon}$ (where $(\mathrm{Id}+B(z))$ is defined and analytic). Using once again the continuity of the trace functional with respect to the trace-norm one finally arrives at $(2.22)$.

As all $B(z), z \in G_{\varepsilon}$, are nuclear and a fortiori compact, $(\operatorname{Id}+B(z))$ is an analytic operator-valued function, normal with respect to $\operatorname{bd}\left(G_{\varepsilon}\right)$ in the sense of [Gohberg/Sigal, 1971, §2]. In particular, Theorem 2.1 (ibid.) says

$$
M\left(\operatorname{Id}+B\left(z_{0}\right)\right)=\frac{1}{2 \pi i} \operatorname{tr} \int_{\left|z-z_{0}\right|=r} B^{\prime}(z)(\operatorname{Id}+B(z))^{-1} d z
$$

where $M\left(\operatorname{Id}+B\left(z_{0}\right)\right)$ is the multiplicity of the singular point $z_{0}$ of $(\operatorname{Id}+B(z))$ (cf. $\S 1$ (ibid.)).The multiplicity $M$ is invariant under equivalence, i.e. if $E(z)$, $F(z)$ are equivalent holomorphic families of invertible operators defined in a neighbourhood of $z_{0}$, then $M\left(E\left(z_{0}\right)\left(\operatorname{Id}+B\left(z_{0}\right)\right) F\left(z_{0}\right)\right)=M\left(\operatorname{Id}+B\left(z_{0}\right)\right)$.

Recall (2.15) for $\alpha=$ Id :

$$
(\operatorname{Id}-z P)=\left(\operatorname{Id}-z P_{\beta}-z \beta P(\operatorname{Id}-\beta)\right)(\operatorname{Id}+B(z))(\operatorname{Id}+A(z))
$$

The first and the last factor on the right-hand side are analytic and invertible in $G_{\varepsilon}$, i.e. $(\operatorname{Id}-z P)$ and $(\operatorname{Id}+B(z))$ are equivalent at $z_{0} \in G_{\varepsilon}$. Hence

$$
M\left(\operatorname{Id}+B\left(z_{0}\right)\right)=M\left(\operatorname{Id}-z_{0} P\right)
$$

and the right-hand side of this equation coincides with the usual notion of (algebraic) multiplicity of the eigenvalue $z_{0}^{-1}$ of $P$ (ibid., $\S 1,1$ ). As $\operatorname{det}_{\mathscr{F}}\left(\mathrm{Id}-z P_{\beta}\right) \neq 0$ for $z \in G_{\varepsilon}$ by (2.18), assertion (b) of the theorem follows now from (2.21), (2.22), (2.24), and (2.25).

2.7. Remark. The theorem allows us to define $\operatorname{det}(\operatorname{Id}-z P)$ as the equivalence class of $\operatorname{det}_{\mathscr{F}}(\operatorname{Id}-z P)$ of analytic functions on $\{|z|<R\}$ under the equivalence $f \sim g$ if and only if $f / g$ and $g / f$ are analytic on $\{|z|<R\}$. This makes particular sense if there is some $\mathscr{F}$ such that $R=r_{\text {as }}=t_{\text {as }}=r_{\text {ess }}$.

\section{Piecewise inVertible Systems AND their MARKOV EXTENSIONS}

\section{A. Piecewise invertible systems.}

Having accomplished the functional analytic prerequisites we now define the class of piecewise invertible dynamical systems to be investigated in the rest of this paper. For similar definitions see [Schweiger, 1975] and [Hofbauer, 1986].

\subsection{Definition. Let $X$ be a topological space.}

(a) The triple $(X, T, \mathscr{Z})$ is a piecewise invertible system, if $\mathscr{Z}$ is a finite or countable partition of $X$ such that for each $Z \in \mathscr{Z} \quad \operatorname{cl}(Z)$ is compact and if the restrictions $T_{\mid Z}$ can be extended to continuous injective maps $T_{Z}: \operatorname{cl}(Z) \rightarrow$ $X$. (cl denotes the topological closure, int the interior, and (for later use) 
bd the boundary.) Often we shall just say system instead of piecewise invertible system.

(b) $(X, T, \mathscr{Z})$ is a piecewise compact system if all $Z \in \mathscr{Z}$ are compact open subsets of $X$.

(c) The system $(X, T, \mathscr{Z})$ is a Markov system if $T Z_{1} \cap Z_{2} \neq \varnothing$ implies $T Z_{1} \supseteq Z_{2}$ for all $Z_{1}, Z_{2} \in \mathscr{Z}$.

3.2. Remark. If $(X, T, \mathscr{Z})$ is piecewise compact, then $T_{Z}=T_{\mid Z}$ is a homeomorphism from $Z$ onto $T Z \quad(Z \in \mathscr{Z})$.

3.3. Definition (Cylinders). For $n \geq 0$ let

$$
\mathscr{Z}_{n}=\bigvee_{i=0}^{n} T^{-i} \mathscr{Z}=\left\{Z_{0} \cap T^{-1} Z_{1} \cap \cdots \cap T^{-n} Z_{n} \neq \varnothing: Z_{i} \in \mathscr{Z}\right\}
$$

be the cylinders of length $n$ of the system $(X, T, \mathscr{Z})$. Denote by $V_{n}(x)$ the cylinder of length $n$ containing $x,(x \in X)$ and by $\operatorname{reg}(X, T, \mathscr{Z})=\{x \in$ $X: T^{k} x \in \operatorname{int} V_{n}\left(T^{k} x\right)$ for all $\left.k \geq 0, n \geq 0\right\}$ the set of all regular points of $(X, T, \mathscr{Z})$. Observe that if $(X, T, \mathscr{Z})$ is piecewise compact, then each $Z \in \mathscr{Z}_{n}$ is compact and $\operatorname{reg}(X, T, \mathscr{Z})=X$.

$(X, T, \mathscr{Z})$ is generating, if $[x]:=\bigcap_{n \geq 0} V_{n}(x)=\{x\}$ for all $x \in X$.

3.4. Definition (Factors, extensions). $(X, T, \mathscr{Z})$ is a factor of $(\hat{X}, \hat{T}, \hat{Z})$, and $(\hat{X}, \hat{T}, \hat{\mathcal{Z}})$ is an extension of $(X, T, \mathscr{Z})$, if there is a continuous map $\pi$ from $\hat{X}$ onto $X$ such that $T \circ \pi=\pi \circ \hat{T}$ on the set $\pi^{-1}(\operatorname{reg}(X, T, \mathscr{Z}))$. Then $T^{k} \circ \pi=\pi \circ \hat{T}^{k}$ for all $k$ on the same set, because this set is $\hat{T}$-invariant. We also say $\pi$-factor or $\pi$-extension if we want to specify the factoring map. Observe that $T \circ \pi=\pi \circ \hat{T}$ on all of $\hat{X}$ if $(X, T, \mathscr{Z})$ is piecewise compact.

\section{B. Canonical extensions.}

Our main goal in this section is to construct in a canonical way a Markov extension for a given piecewise compact system. At the end we also show how to find, in a canonical way, a piecewise compact extension for an arbitrary system.

From now on let $(X, T, \mathscr{Z})$ be a piecewise compact system. The ideas behind the following construction of a Markov extension are borrowed from [Hofbauer, 1986] and some of his earlier works, e.g. [Hofbauer, 1979]. References can be found in his 1986 paper. Analogous constructions for (two-sided) sofic systems are carried out in [Krieger, 1984].

3.5. Definition (Successors). Suppose $C$ is a compact subset of $X$. The nonempty sets among $T C \cap Z$ with $Z \in \mathscr{Z}$ are called the successors of $C$. We write $C \rightarrow D$ if $D=T C \cap Z$. As all $Z \in \mathscr{Z}$ are assumed to be compact, successors of compact sets are again compact, and one can iterate the formation of successors. Let $\mathscr{D}$ be the smallest set of (compact) subsets of $X$ that contains $\mathscr{Z}$ and is closed under forming successors.

The following lemma relates the above construction to cylinders. Its proof is simple and can be found in [Hofbauer, 1986, Lemma 1]. 
3.6. Lemma. Suppose that $Z_{i} \in \mathscr{Z}(i=0, \ldots, k)$ and that $D \subseteq Z_{0}$. Let $A_{k}=D \cap \bigcap_{i=1}^{k} T^{-i} Z_{i}$ and $D_{0}=D, D_{i}=T\left(D_{i-1}\right) \cap Z_{i} \quad(i=1, \ldots, k)$. Then $D_{k}=T^{k} A_{k}$ and $A_{k}=\bigcap_{i=0}^{k} T^{-i} D_{i}$. If $D=Z_{0}$, then $A_{k} \in \mathscr{Z}_{k}$.

Now let

$$
\hat{X}=\bigcup_{D \in \mathscr{D}} \hat{D}
$$

where the $\hat{D}$ are pairwise disjoint copies of the sets $D \in \mathscr{D}$. Elements of $\hat{X}$ are denoted by $\hat{x}=(x, D)$ with $x \in D \in \mathscr{D}$. Define $\hat{T}: \hat{X} \rightarrow \hat{X}$ by

$$
\hat{T}(x, C)=(T x, D) \text { if } C \rightarrow D \text { and } T x \in D
$$

and let $\hat{Z}=\{\hat{D}: D \in \mathscr{D}\}$. Then $(\hat{X}, \hat{T}, \hat{Z})$ is, by construction, a piecewise compact Markov system. It is a $\pi$-extension of $(X, T, \mathscr{Z})$ where $\pi: \hat{X} \rightarrow X$ is defined by $\pi(x, C)=x$. By $\pi_{\mathscr{D}}$ we denote the projection from $\hat{X}$ to $\mathscr{D}$, $\pi_{\mathscr{D}}(x, C)=C$.

3.7. Definition. (a) $(\hat{X}, \hat{T}, \hat{\mathcal{Z}})$ is the canonical Markov extension of $(X, T, \mathscr{Z})$.

(b) The Markov diagram of $(X, T, \mathscr{Z})$ is the directed graph $(\mathscr{D}, \rightarrow)$ with vertex-set $\mathscr{D}$ and edges defined by the successor relation (cf. [Hofbauer, 1986]). As usual it can be represented by a $\{0,1\}$-valued $\mathscr{D} \times \mathscr{D}$-matrix. ${ }^{14}$

(c) The Markov-diagram is uniformly forward finite if there is a uniform bound on the number of successors of a single vertex.

3.8. Remark. If $(X, T, \mathscr{Z})$ is a piecewise compact Markov system, it coincides with its canonical Markov extension, and the Markov diagram describes the Markov structure of $(X, T, \mathscr{Z})$ itself.

3.9. Definition (Orbits and paths). An orbit of length $n$ (in $X$ ) is an ordered $n$-tuple $x, T x, \ldots, T^{n-1} x$. It is periodic if $T^{n} x=x$. Orbits in $\hat{X}$ are defined analogously. A path of length $n$ (in $\mathscr{D}$ ) is an ordered $(n+1)$-tuple $\omega=$ $D_{0} D_{1} \ldots D_{n}$ with $D_{i-1} \rightarrow D_{i} \quad(i=1, \ldots, n)$. It is closed, if $D_{n}=D_{0}$. With $\omega$ we associate the cylinder of length $n \quad Z(\omega)=\bigcap_{i=0}^{n} T^{-i} D_{i}$. Infinite orbits and paths are defined analogously.

Note that Lemma 3.6 establishes a $1-1$ correspondence $\omega \leftrightarrow \mathscr{Z}(\omega)$ between paths and cylinders of length $n$. The following relation between orbits in $\hat{X}$ and paths in $\mathscr{D}$ are implicit in Theorem 1 of [Hofbauer, 1986]. The proof is easy.

3.10. Lemma. Suppose $(X, T, \mathscr{Z})$ is a piecewise compact system.

(a) Each $\hat{x} \in \hat{X}$ induces a unique infinite path $\omega(\hat{x})=D_{0} D_{1} D_{2} \cdots$ by $D_{j}=$ $\pi_{\mathscr{g}}\left(\hat{T}^{j} \hat{x}\right)(j=0,1,2, \ldots)$. Notation: $\omega_{n}(\hat{x})=D_{0} \cdots D_{n}$.

\footnotetext{
${ }^{14}$ Hofbauer investigates the topological Markov chain defined by this matrix. It is again a piecewise compact system and can be obtained as an $\omega$-factor of $(\hat{X}, \hat{T}, \hat{Z})$ by $\omega(\hat{x})=\left(\pi_{\mathscr{X}}\left(\hat{T}^{n} \hat{x}\right)\right)_{n \geq 0}$. If $(X, T, \mathscr{Z})$ is generating, then $\omega$ is a homeomorphism between $\hat{X}$ and the chain, cf. Lemma
} 3.6. 
(b) Each infinite path $D_{0} D_{1} D_{2} \cdots$ in the Markov diagram represents a point $\hat{x} \in \hat{X}$, i.e. there is $\hat{x} \in \hat{X}$ with $\omega(\hat{x})=D_{0} D_{1} D_{2} \cdots$. This $\hat{x}$ is unique if $[x]=\{x\}$, in particular if $(X, T, \mathscr{Z})$ is generating.

(c) If $\omega(\hat{x})=C_{0} C_{1} C_{2} \cdots, \omega(\hat{y})=D_{0} D_{1} D_{2} \cdots, \pi(\hat{x})=\pi(\hat{y})=x$ for $\hat{x}, \hat{y} \in \hat{X}$, and if $C_{0} \cap V_{k}(x)=D_{0} \cap V_{k}(x)$ for some $k \geq 0$, then $C_{i}=D_{i}$ $(i \geq k)$.

The following lemma, which relates periodic orbits in $X$ and $\hat{X}$ to closed paths in $\mathscr{D}$, is a slight variation of Theorem 8 in [Hofbauer, 1986]:

3.11. Lemma. Suppose $(X, T, \mathscr{Z})$ is a generating, piecewise compact system.

(a) If $D_{0} D_{1} \ldots D_{n-1} D_{0}$ is a closed path, then there is exactly one $x \in D_{0}$ such that $\hat{T}^{i}\left(x, D_{0}\right)=\left(T^{i \bmod n} x, D_{i \bmod n}\right)$ for $i=0,1,2, \ldots$.

(b) If $T^{n} x=x$ for some $x \in X$, and if $x \in \operatorname{int}\left(\pi_{\mathscr{D}} \circ \hat{T}^{n}\left(x, V_{0}(x)\right)\right)$, then there is $\hat{x} \in \pi^{-1}\{x\}$ with $\hat{T}^{n} \hat{x}=\hat{x}$. If $x$ is in the interior of each $D \in \mathscr{D}$ it belongs to, then $\hat{x}$ is unique.

Proof. (a) By Lemma 3.6, $A_{k}=\bigcap_{i=0}^{k} T^{-i} D_{i \bmod n}$ is nonempty and compact for all $k \in \mathbb{N}$. Hence $A_{\infty}=\bigcap_{i=0}^{\infty} T^{-i} D_{i \bmod n} \subseteq D_{0}$ is nonempty. Let $x \in A_{\infty}$. By Lemma 3.6, $A_{\infty} \subseteq[x]=\{x\}$, and we obtain inductively $\hat{T}\left(T^{i} x, D_{i}\right)=$ $\left(T^{i+1} x, D_{(i+1) \bmod n}\right)(i=0, \ldots, n-1)$. Since $\left\{T^{n} x\right\}=T^{n} A_{\infty} \subseteq A_{\infty}=\{x\}$, this yields

$$
\hat{T}^{i}\left(x, D_{0}\right)=\left(T^{i \bmod n} x, D_{i \bmod n}\right) \quad(i \geq 0) .
$$

As each point satisfying this relation belongs to $A_{\infty}=\{x\}, x$ is uniquely determined.

(b) Let $Z=V_{0}(x)$. Then $\pi(x, Z)=x=\pi\left(\hat{T}^{n}(x, Z)\right)$, and for some $k$ big enough holds

$$
\pi_{\mathscr{Z}}(x, Z) \cap V_{k}(x)=V_{k}(x)=\pi_{\mathscr{D}}\left(\hat{T}^{n}(x, Z)\right) \cap V_{k}(x)
$$

because $(X, T, \mathscr{Z})$ generates. Hence $\pi_{\mathscr{D}}\left(\hat{T}^{i}(x, Z)\right)=\pi_{\mathscr{D}}\left(\hat{T}^{n+i}(x, Z)\right)$ for $i \geq$ $k$ by Lemma $3.10(\mathrm{c})$, and for a suitable multiple $\ln$ of $n$ and $\hat{x}:=\hat{T}^{l n}(x, Z)$ holds

$$
\begin{aligned}
\hat{T}^{n} \hat{x} & =\left(T^{(l+1) n} x, \pi_{\mathscr{Q}}\left(\hat{T}^{(l+1) n}(x, Z)\right)\right) \\
& =\left(T^{l n} x, \pi_{\mathscr{D}}\left(\hat{T}^{l n}(x, Z)\right)\right) \\
& =\hat{x} \in \pi^{-1}\{x\}
\end{aligned}
$$

and $\omega_{n}(\hat{x})$ is closed, i.e. $\omega(\hat{x})$ is periodic.

If $\hat{y}$ is another point with $\pi(\hat{y})=x, \hat{T}^{n} \hat{y}=\hat{y}$, then also $\omega(\hat{y})$ is periodic, and by Lemma 3.10 (c) the two paths coincide if $x$ is in the interior of each $D \in \mathscr{D}$ it belongs to. $\hat{x}=\hat{y}$ follows now from part (a). 
3.12. Example I (topological Markov shifts, sofic systems).

Let $\Sigma$ be a finite or countable set of symbols, $A$ a $\{0,1\}$-valued $\Sigma \times \Sigma$-matrix which is row-finite (i.e. $\sum_{\tau \in \Sigma} A(\sigma, \tau)<\infty$ for all $\sigma \in \Sigma$ ), and let

$$
\Omega_{A}=\left\{\left(\sigma_{1} \sigma_{2} \sigma_{3} \cdots\right) \in \Sigma^{\mathbb{N}}: A\left(\sigma_{i}, \sigma_{i+1}\right)=1 \text { for all } i \in \mathbb{N}\right\}
$$

With the product topology of the discrete topology on $\Sigma$ the shift space $\Omega_{A}$ is a topological space. If $S: \Omega_{A} \rightarrow \Omega_{A}, S\left(\sigma_{1} \sigma_{2} \sigma_{3} \cdots\right)=\left(\sigma_{2} \sigma_{3} \sigma_{4} \cdots\right)$ denotes the shift transformation, and if $\mathscr{Z}=\left\{[\sigma]_{1}: \sigma \in \Sigma\right\}$ (where $[\sigma]_{1}=\left\{\left(\sigma_{1} \sigma_{2} \sigma_{3} \cdots\right) \in\right.$ $\left.\left.\Omega_{A}: \sigma_{1}=\sigma\right\}\right)$ is the partition into cylinders of length 1 , Then $\left(\Omega_{A}, S, \mathscr{Z}\right)$ is a generating, piecewise compact system. (For the compactness of the fibers see Lemma 1.5(b) in [Wagoner, 1988].) We have $\mathscr{D}=\mathscr{Z}$ because of the Markov structure of $\Omega_{A}$, and it is easily seen that $\left(\Omega_{A}, S, \mathscr{Z}\right)$ is its own canonical Markov extension.

If $\Sigma$ is finite, if $\tilde{\Sigma}$ is another alphabet, and if $\theta: \Sigma \rightarrow \tilde{\Sigma}$, then $\theta$ extends coordinate-wise to a map from $\Omega_{A}$ to $\tilde{\Sigma}^{N}$, and

$$
\Omega_{A, \theta}=\left\{\theta(\omega): \omega \in \Omega_{A}\right\}
$$

is a sofic system (see [Coven/Paul, 1975]). As in [Krieger, 1984] it is easy to show that $\mathscr{D}$ is finite.

\subsection{Example II (up-and-down-counter).}

This is an example of a family of closed subshifts of $\{0,1\}^{N}$ which are not sofic. Before I describe these systems, some notational conventions are introduced which will be used later, too.

For a set $\Sigma$ of symbols let $\Sigma^{+}=\left\{\sigma_{1} \cdots \sigma_{n}: n \geq 1, \sigma_{i} \in \Sigma\right\}$ and $\Sigma^{*}=\Sigma^{+} U$ $\{$ empty word $\}$. Similarly, for $W \subseteq \Sigma^{*}$ let $W^{+}=\left\{w_{1} \cdots w_{n}: n \geq 1, w_{i} \in W\right\}$ and $W^{*}=W^{+} \cup\{$ empty word $\}$. Furthermore let $W^{\infty}=\left\{w_{1} w_{2} w_{3} \cdots: w_{i} \in\right.$ $W\} . W^{\infty}$ is considered as a subset of $\Sigma^{\mathbf{N}}$. By $\Omega_{0}(W)$ we denote the smallest shift-invariant subset of $\Sigma^{N}$ containing $W^{\infty}$ and by $\Omega(W)$ its topological closure in $\Sigma^{\mathbf{N}}$.

If $A \subseteq \Sigma^{*}$ and $B \subseteq \Sigma^{*}$ or $B \subseteq \Sigma^{N}$, we let $A B=\{u v: u \in A, v \in B\}$. $A B \subseteq \Sigma^{*}$ or $\Sigma^{N}$, respectively.

If $\sigma \in \Sigma$ then $\sigma^{n}=\sigma \cdots \sigma$ ( $n$-times).

Here comes the up-and-down-counter: Let $\Sigma=\{0,1\}$, fix $M \subseteq \mathbb{N}$ (infinite, otherwise we would come out with a sofic system), and let

$$
W_{M}=\left\{0^{i} 1^{i}: i \in M\right\}
$$

and

$$
V_{M}=\left\{0^{i} 1^{j}: 0 \leq i \leq j<\infty \text { and } j \in M \text { if } i>0\right\}
$$

It is quite obvious that $\Omega_{0}\left(W_{M}\right)=V_{M} W_{M}^{\infty}$, and it is also not hard to see that $\Omega\left(W_{M}\right)=V_{M} R_{M}$ where

$$
R_{M}=W_{M}^{\infty} \cup W_{M}^{*}\left\{0^{\infty}\right\} \cup\left\{1^{\infty}\right\}
$$


If $S: \Omega\left(W_{M}\right) \rightarrow \Omega\left(W_{M}\right)$ denotes again the shift-transformation and if $\mathscr{Z}=$ $\left\{[0]_{1},[1]_{1}\right\}$, then $\left(\Omega\left(W_{M}\right), S, \mathscr{Z}\right)$ is a generating, piecewise compact system, and its Markov diagram has the following structure, if, for example, the three smallest members of $M$ are 2,5, and 6:

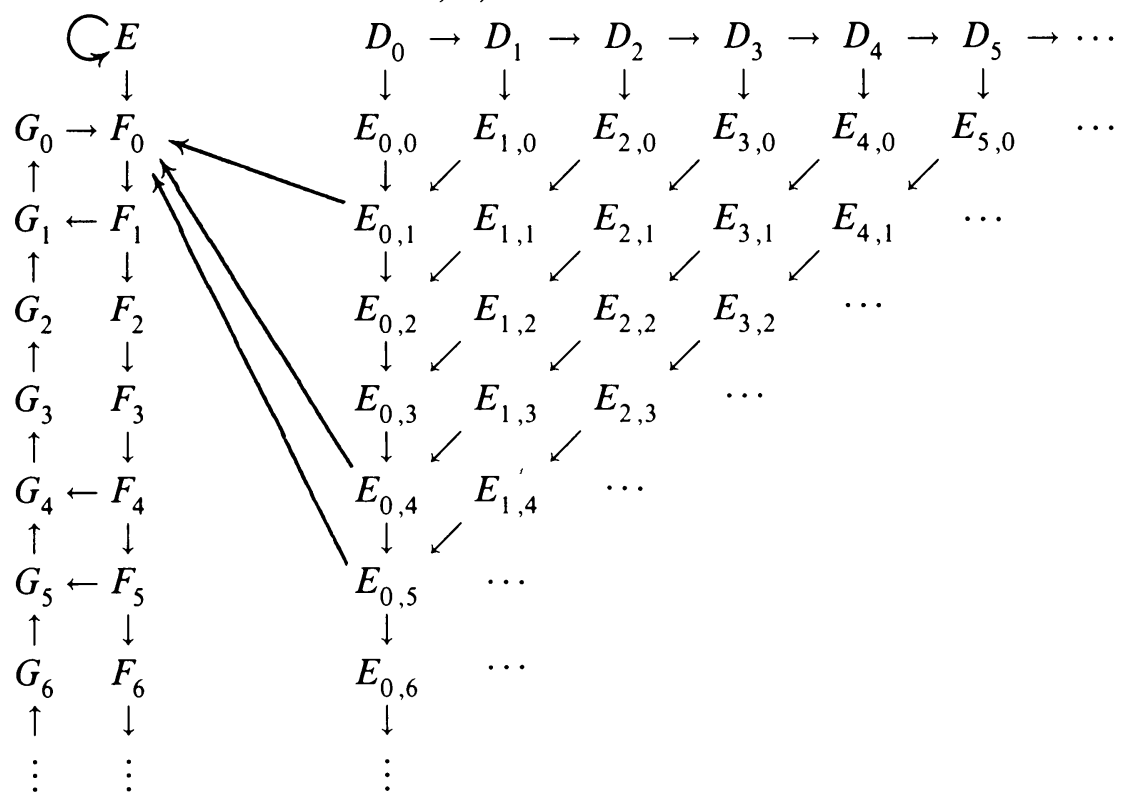

Here

$$
\begin{aligned}
D_{n} & =\left\{0^{i} 1^{j}: 1 \leq i \leq j-n, j \in M\right\} R_{M} \cup\left\{0^{\infty}\right\}, \\
E_{n, m} & =\left\{1^{j}: j \geq n+1, j+m \in M\right\} R_{M}, \\
E & =\left\{1^{j}: 1 \leq j<\infty\right\} R_{M}=[1]_{1}, \\
F_{0} & =R_{M} \backslash\left\{1^{\infty}\right\}, \\
F_{n} & =\left\{0^{j-n} 1^{j}: n<j<\infty, j \in M\right\} F_{0} \cup\left\{0^{\infty}\right\} \quad \text { for } n \geq 1, \\
G_{n} & =\left\{1^{n+1}\right\} F_{0} .
\end{aligned}
$$

For special choices of $M$ the right-hand side of the diagram may collapse. For $M=\mathbb{N}$, for example, $E_{n, m}=E_{n, 0}$ for all $m$ and $E_{0,0}=E$.

If $M$ is finite, the same construction yields a finite diagram, and $\left(\Omega\left(W_{M}\right), S\right)$ is a one-block factor of the topological Markov chain defined by $(\mathscr{D}, \rightarrow)$, i.e. it is sofic. For later use we note some further facts:

(a) All closed paths are of the form $F_{0} \cdots F_{j} G_{j} \cdots G_{0} F_{0} \quad(j \in M)$ (or translates of these cycles), except for the trivial cycle $E E$.

(b)

$$
\begin{gathered}
D_{0}=\operatorname{int}\left(D_{0}\right), \quad D_{n} \backslash \operatorname{int}\left(D_{n}\right)=\left\{0^{\infty}\right\} \quad(n \geq 1), \\
E=\operatorname{int}(E), \quad E_{n, m} \backslash \operatorname{int}\left(E_{n, m}\right)=\left\{1^{\infty}\right\} \quad(\text { if } M \neq \mathbb{N}), \\
F_{n} \backslash \operatorname{int}\left(F_{n}\right)=\left\{0^{\infty}\right\}, \quad G_{n}=\operatorname{int}\left(G_{n}\right) .
\end{gathered}
$$




\subsection{Extensions for nonpiecewise compact systems.}

The relations between $(X, T, \mathscr{Z})$ and its canonical Markov extension as stated in Lemmas 3.10 and 3.11 are no longer true, if $(X, T, \mathscr{Z})$ is not piecewise compact. In order to investigate a nonpiecewise compact system $(\tilde{X}, \tilde{T}, \tilde{Z})$ by means of a Markov extension it is therefore useful to construct first a (usually non-Markovian) piecewise compact extension $(X, T, \mathscr{Z})$ of that system. This can be done as follows:

Suppose that $\tilde{X}$ is compact and that $V \in \tilde{Z}$. Let

$$
X_{0}=\tilde{X}, \quad X_{n}=\sum_{Z \in \dot{\mathscr{X}}_{n-1}} \operatorname{cl}(Z), \quad V_{n}=\sum_{\substack{Z \in \dot{X}_{n-1} \\ Z \subseteq v^{\prime}}} \operatorname{cl}(Z) \quad(n \geq 1),
$$

(disjoint topological sum), and let $T_{n}: X_{n} \rightarrow X_{n-1}$ be the continuous extension of $\tilde{T}$ to $X_{n}(n \geq 1)$. Denote by $\Phi_{k, n}$ the canonical projection $X_{n} \rightarrow X_{k} \quad(n \geq$ $k)$ and by $\left(X_{\infty}, \Phi_{k, \infty}\right)$ the inverse limit of the inverse spectrum $\left(X_{n}, \Phi_{k, n}\right)$ (see Appendix 2, Definition 2.2 of [Dugundji, 1966]). Similarly $\left(V_{\infty}, \Phi_{k, \infty \mid V_{\infty}}\right)$ is the inverse limit of $\left(V_{n}, \Phi_{k, n \mid V_{n}}\right)$. The following diagram commutes:

$$
\begin{aligned}
& X_{0} \stackrel{\Phi}{\longleftarrow} X_{1} \stackrel{\Phi}{\longleftarrow} X_{1} \stackrel{\Phi}{\longleftarrow} \cdots \stackrel{\Phi}{\longleftarrow} X_{k-1} \stackrel{\Phi}{\longleftarrow} X_{\infty} \\
& \uparrow T_{1 \mid r_{1}} \quad \uparrow T_{2 \mid r_{2}} \quad \uparrow T_{3 \mid r_{3}} \quad \uparrow T_{k \mid r_{k}} \quad \uparrow T_{\infty \mid r_{\infty}} \\
& V_{1} \stackrel{\Phi}{\longleftarrow} V_{2} \stackrel{\Phi}{\longleftarrow} V_{3} \stackrel{\Phi}{\longleftarrow} \cdots \stackrel{\Phi}{\longleftarrow} V_{k} \stackrel{\Phi}{\longleftarrow} V_{\infty}
\end{aligned}
$$

The map $T_{\infty}$ is uniquely defined by the $T_{k} \quad(1 \leq k \leq \infty)$, and as all $T_{k \mid V_{k}}$ $(1 \leq k<\infty)$ are continuous and injective, the same holds for $T_{\infty \mid V_{\infty}}$.

Hence $\left(X_{\infty}, T_{\infty}, \Phi_{0, \infty}^{-1} \tilde{\mathscr{Z}}\right)$ is a piecewise compact $\Phi_{0, \infty}$-extension of $(\tilde{X}, \tilde{T}, \tilde{Z})$. Properties analogous to Lemmas 3.10 and 3.11 must be deduced separately in particular cases from the above construction. They depend strongly on how far $(\tilde{X}, \tilde{T}, \tilde{Z})$ is from being piecewise compact.

For later use we note that

$$
\Phi_{0, \infty}\left(T_{\infty}^{k} x\right)=\tilde{T}^{k}\left(\Phi_{0, \infty} x\right) \quad \text { for } x \in \Phi_{0, \infty}^{-1}(\operatorname{reg}(\tilde{X}, \tilde{T}, \tilde{Z}))
$$

(see Definition 3.3) and for all $x$ if $\tilde{T}$ is continuous.

We close this section with a class of examples for nonpiecewise compact systems:

3.15. Example III (piecewise monotonic transformations).

Let $\tilde{X} \subseteq \mathbf{R} \cup\{+\infty,-\infty\}$ be a bounded or unbounded interval, $\tilde{Z}$ a finite or countable partition of $\tilde{X}$ into intervals, and let $\tilde{T}: \tilde{X} \rightarrow \tilde{X}$ be a map for which $\tilde{T}_{\mid Z}$ is strictly monotone and continuous for each $Z \in \tilde{Z}$.

(a) The $\beta$-transformation, $\beta>1: \tilde{X}=[0,1], \tilde{T} x=\beta x \bmod 1, \tilde{Z}=$ $\left\{\left[0, \beta^{-1}\right),\left[\beta^{-1}, 2 \beta^{-1}\right), \ldots,\left[k \beta^{-1}, 1\right]\right\}$ where $k$ is the integer part of $\beta$. In 
this case

$$
\begin{aligned}
X_{1}= & \sum_{j=0}^{k-1}\left[j \beta^{-1},(j+1) \beta^{-1}\right]+\left[k \beta^{-1}, 1\right] \\
X_{2}= & \sum_{i=0}^{k-1} \sum_{j=0}^{k-1}\left[i \beta^{-1}+j \beta^{-2}, i \beta^{-1}+(j+1) \beta^{-2}\right] \\
& +\sum_{i=0}^{k-1}\left[i \beta^{-1}+k \beta^{-2},(i+1) \beta^{-1}\right] \\
& +\sum_{j=0}^{l-1}\left[k \beta^{-1}+j \beta^{-2}, k \beta^{-1}+(j+1) \beta^{-2}\right] \\
& +\left[k \beta^{-1}+l \beta^{-2}, 1\right],
\end{aligned}
$$

where $l$ is the integer part of $\beta(\beta-k)$, and so on. The Markov diagram looks like

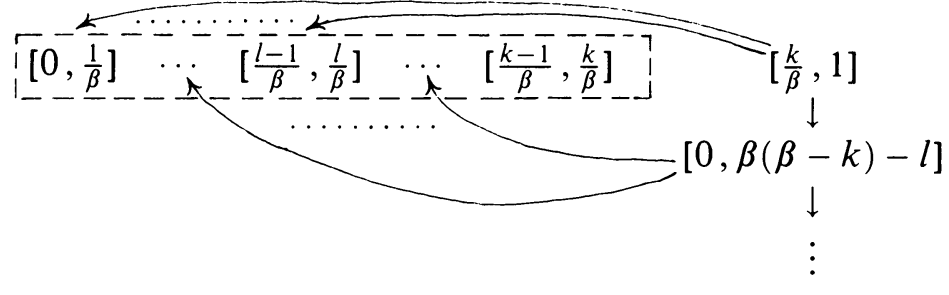

where also all intervals in the dashed box are pairwise joined by arrows.

(b) The continued fraction transformation:

$\tilde{X}=[0,1], \quad \tilde{T} x=\frac{1}{x} \bmod 1(x>0)$ and $\tilde{T}(0)=0$. Obviously $\mathscr{D}=$ $\left\{I_{n}=\left[\frac{1}{n+1}, \frac{1}{n}\right]: n \in \mathbb{N}\right\} \cup\{\{0\}\}$, and there are arrows $I_{n} \rightarrow I_{m}, I_{n} \rightarrow\{0\}$, and $\{0\} \rightarrow\{0\}$ for all $m, n \in \mathbb{N}$. We shall not pursue further such systems whose Markov diagrams are not uniformly forward finite. ${ }^{15}$

\section{TRANSFER OPERATORS AND ZETA FUNCTIONS}

\section{A. Transfer operators.}

In this section we take a first step from the purely topological concepts of the last section towards a consideration of "smooth" and measure-theoretical structures of our systems:

Let $(X, T, \mathscr{Z})$ be a piecewise invertible system. By $\operatorname{mb}(X)$ (or simply $\mathrm{mb}$ if no confusion can occur) we denote the space of complex valued, bounded, Borel-measurable functions on $X$. Pick some $g \in \operatorname{mb}(X)$, and fix the following notation:

$$
g_{n}(x)=g(x) \cdot g(T x) \cdot \ldots \cdot g\left(T^{n-1} x\right)
$$

\footnotetext{
$15 \mathrm{Cf}$. however the last paragraph of Example 4.10 and Remark 6.3.
} 
4.1. Definition. (a) $(X, T, \mathscr{Z}, g)$ is a weighted system with weight function $g$, if

$$
\sum_{T x=y}|g(y)| \in \operatorname{mb}(X)
$$

as a function of $x$.

(b)

$$
g_{\infty}=\lim _{n \rightarrow \infty}\left(\left\|g_{n}\right\|_{\infty}\right)^{1 / n}
$$

where $\|\cdot\|_{\infty}$ is the supremum-norm on $\mathrm{mb}(X)$.

With a weighted system $(X, T, \mathscr{Z}, g)$ we associate the linear operator $P_{g}: \mathrm{mb} \rightarrow \mathrm{mb}$ (sometimes abbreviated as $P$ )

$$
P_{g} f(x)=\sum_{T y=x} g(y) f(y) .
$$

Our goal is to restrict $P_{g}$ to suitable subspaces of smooth functions and to show that under certain assumptions on $T$ and $g$ this restriction is quasicompact or even quasinuclear.

For later use we note

$$
P_{g}^{n} f(x)=\sum_{T^{n} y=x} g_{n}(y) f(y) \quad(n \in \mathbb{N})
$$

4.2. Definition. $P_{g}$ is the transfer operator of $(X, T, \mathscr{Z}, g)$.

4.3. Remark. These operators are frequently called Perron-Frobenius operators, in particular if the following setting is considered:

There is a nonatomic measure $m$ on $X$ with respect to which $T$ is nonsingular and such that $g^{-1}$ is its $m$-derivative. ${ }^{16}$ Then $P_{g}$ is dual to $T$ in the sense that

$$
\int_{X} P_{g} f_{1} \cdot f_{2} d m=\int_{X} f_{1} \cdot T f_{2} d m
$$

whenever both sides of this equation are well defined $(T f:=f \circ T)$. Obviously $m(\{g=0\})=0$.

It is easily checked that for $0 \leq f \in \mathrm{mb}$ and $\mu=f \cdot m$

$$
P_{g} f=f \quad \text { if and only if } \quad T \mu=\mu,
$$

where $(T \mu)(A)=\mu\left(T^{-1} A\right)$. But even if such an absolutely continuous, $T$ invariant measure exists, this is not the only choice of $g$ for which the operator $P_{g}$ describes relevant aspects of the dynamics of $(X, T, \mathscr{Z})$ (cf. §9.C).

We note that (4.3) implies

$$
\int_{X}\left|P_{g} f\right| d m \leq \int_{X}|f| d m \quad\left(f \in L_{m}^{1}\right) .
$$

\footnotetext{
${ }^{16}$ I.e. $\int_{A} g^{-1} d m=m(T A)$ for all measurable $A$ contained in some $Z \in \mathscr{Z}$.
} 
4.4. Remark. One might ask if, with $g$ as in the preceding remark, $P_{g}: L_{m}^{1} \rightarrow$ $L_{m}^{1}$ has already interesting spectral properties. This is not the case:

Assume for simplicity that $P 1=1$, i.e. $T m=m$ and $P(T f)=f$. For $\lambda \in \mathbb{C},|\lambda|<1$, define $Q_{\lambda}: L_{m}^{1} \rightarrow L_{m}^{1}$ by

$$
Q_{\lambda}=\sum_{k=0}^{\infty} \lambda^{k} T^{k}(\mathrm{Id}-T P)
$$

Then $P Q_{\lambda}=\lambda Q_{\lambda}, Q_{\lambda}^{2}=Q_{\lambda}$, and $Q_{\lambda} f=f$ if and only if $P f=\lambda f$. Hence $Q_{\lambda}$ is a projection onto $\left\{f \in L_{m}^{1}: P f=\lambda f\right\}$, and $\lambda$ is a $L_{m}^{1}$-eigenvalue of $P$ if and only if $Q_{\lambda} \not \equiv 0$. Since $T$ is not a bijection, there is $0 \neq f \in L_{m}^{1}$ with $0 \neq f-T P f=P^{k} T^{k}(f-T P f)$, i.e. $T^{k}(f-T P f) \neq 0$ for all $k$, and there are arbitrarily large $n$ such that

$$
Q_{\lambda}^{(n)} f=\sum_{k=0}^{n-1} \lambda^{k} T^{k}(\mathrm{Id}-T P) f \neq 0 .
$$

Now $Q_{\lambda} f \neq 0$ follows from

$$
\begin{gathered}
Q_{\lambda} f=Q_{\lambda}^{(n)} f+\sum_{k=1}^{\infty} \lambda^{k n} T^{k n} Q_{\lambda}^{(n)} f \text { and } \\
\left\|\sum_{k=1}^{\infty} \lambda^{k n} T^{k n} Q_{\lambda}^{(n)} f\right\|_{1} \leq \frac{|\lambda|^{n}}{1-|\lambda|^{n}}\left\|Q_{\lambda}^{(n)} f\right\|_{1} .
\end{gathered}
$$

Hence $\lambda$ is a $L_{m}^{1}$-eigenvalue of $P$, and the $L_{m}^{1}$-spectrum of $P$ is the whole unit-disc. (This argument is taken from $\S I V$ in [Keller, 1984]. See also $\S 1$ of [Takahashi, 1981].)

\section{B. Zeta-functions.}

Another object we associate with $(X, T, \mathscr{Z}, g)$ is the zeta function $\zeta_{g}(z)$ (or simply $\zeta(z))$ :

4.5. Definition. For $Y \subseteq X$ and $\mathscr{Y} \subseteq \mathscr{Z}$ let

$$
\gamma(Y, n)=\sum_{\substack{x: T^{n}, n_{1}=1 \\ T^{\prime} x \in Y^{\prime} 0 \leq i \leq n}} g_{n}(x) \quad \text { and } \quad \gamma(\mathscr{Y}, n)=\gamma\left(\bigcup_{Z \in \mathscr{Y}} Z, n\right)
$$

and define

$$
\zeta_{g}(Y, z)=\exp \left(\sum_{n=1}^{\infty} \frac{z^{n}}{n} \gamma(Y, n)\right), \quad \zeta_{g}(z)=\zeta_{g}(X, z) .
$$

Let

$$
\gamma(\mathscr{Y})=\varlimsup_{n \rightarrow \infty}|\gamma(\mathscr{Y}, n)|^{1 / n}
$$


Then $\zeta_{g}(z)$ is analytic in $\left\{|z|<\gamma(\mathscr{Z})^{-1}\right\}$, and using results of $\S 2$, we shall see that, under the same conditions we need in order to show that $P_{g}$ is quasinuclear on some suitable function space, $\zeta_{g}(z)$ can be extended to a meromorphic function on a bigger domain, where its inverse is closely related to the Fredholm determinant of $P_{g}$.

\section{Transfer operators and zeta functions for Markov extensions.}

Suppose $(X, T, \mathscr{Z})$ is a piecewise compact system and $(\hat{X}, \hat{T}, \hat{Z})$ its canonical Markov extension. A weight function $g$ is lifted by the canonical projection $\pi: \hat{X} \rightarrow X$ to a weight function $\hat{g} \in \operatorname{mb}(\hat{X}):$

$$
\hat{g}(\hat{x})=g(\pi(\hat{x})) .
$$

By $\hat{P}=\hat{P}_{\hat{g}}$ and $\hat{\zeta}(z)=\hat{\zeta}_{\hat{g}}(z)$ we denote the associated transfer operator and zeta function on $\hat{X}$.

A function $\hat{f} \in \mathrm{mb}(\hat{X})$ can also be regarded as a family $\left(f_{D}\right)_{D \in \mathscr{D}}$ of functions $f_{D}: D \rightarrow \mathbb{C}$ where $f_{D}(x)=\hat{f}(x, D)$. With this notation $\hat{P}_{\hat{g}}$ can be written in terms of the Markov diagram:

$$
\left(\hat{P}_{\hat{g}}^{n} \hat{f}\right)_{D}=\sum_{\substack{C_{0} \ldots, C_{n-1} \in \mathscr{S} \\ c_{0} \rightarrow \cdots \rightarrow C_{n-1} \rightarrow D}}\left(f_{C_{0}} \cdot g_{C_{0}}\right) \circ\left(T_{\mid C_{0}}^{-1} \circ \cdots \circ T_{\mid C_{n-1}}^{-1}\right)_{\mid D}
$$

Similarly, the zeta function $\hat{\zeta}$ can be expressed in terms of closed paths of the Markov diagram, provided $(X, T, \mathscr{Z})$ generates:

Suppose $\omega=D_{0} \cdots D_{n-1} D_{0}$ is a closed path of length $n$ in $\mathscr{D}$. By Lemma $3.11(\mathrm{a})$ there is exactly one $x \in D_{0}$ such that $\hat{T}^{i}\left(x, D_{0}\right)=$ $\left(T^{i \bmod n} x, D_{i \bmod n}\right)(i \geq 0)$, and we can define $\hat{g}_{n}(\omega)=\hat{g}_{n}\left(x, D_{0}\right)$. Observing Lemmas 3.10(a) and 3.11(a) we thus obtain

$$
\hat{\zeta}_{\hat{g}}(z)=\exp \left(\sum_{n=1}^{\infty} \frac{z^{n}}{n} \sum_{\omega \text { closed path of length } n} \hat{g}_{n}(\omega)\right) .
$$

The linear systems $\left(\mathrm{mb}(X), P_{g}\right)$ and $\left(\mathrm{mb}(\hat{X}), \hat{P}_{\hat{g}}\right)$ are related by the maps

$$
\begin{gathered}
e_{*}: \operatorname{mb}(X) \rightarrow \operatorname{mb}(\hat{X}), \\
\left(e_{*} f\right)(x, C)= \begin{cases}f(x), & \text { if } C \in \mathscr{Z}, \\
0, & \text { if } C \in \mathscr{D} \backslash \mathscr{Z}\end{cases}
\end{gathered}
$$

and

$$
\pi_{*}: \operatorname{mb}(\hat{X}) \rightarrow \operatorname{mb}(X), \quad\left(\pi_{*} \hat{f}\right)(x)=\sum_{\pi(\hat{x})=x} \hat{f}(\hat{x}) .
$$

Observe that

$$
\pi_{*}\left(e_{*} f\right)=f
$$


The basic (but not the only) device to "project" spectral properties from $\hat{P}$ down to $P$ is

4.6. Lemma. The linear system $\left(\mathrm{mb}(X), P_{g}\right)$ is a factor of the linear system $\left(\mathrm{mb}(\hat{X}), \hat{P}_{\hat{g}}\right)$ via the onto-homomorphism $\pi_{*}$, i.e.

In particular

$$
\pi_{*} \circ \hat{P}_{\hat{g}}=P_{g} \circ \pi_{*}
$$

$$
P_{g}^{n} f=\pi_{*} \hat{P}_{\hat{g}}^{n}\left(e_{*} f\right) \text {. }
$$

The proof is left to the reader. For similar statements cf. Lemma 10 in [Hofbauer/Keller, 1984] and Lemma 1 in [Mayer, 1984].

Denote by $\pi_{n}$ the restriction of $\pi$ to the set $\left\{\hat{x} \in \hat{X}: \hat{T}^{n} \hat{x}=\hat{x}\right\}$. The next lemma relates $\zeta_{g}$ and $\hat{\zeta}_{\hat{g}}$ :

\subsection{Lemma.}

$$
\hat{\zeta}_{\hat{g}}(z)=\zeta_{g}(z) \cdot \exp \left(\sum_{n=1}^{\infty} \frac{z^{n}}{n} \sum_{T^{n} x=x} g_{n}(x)\left(\operatorname{card} \pi_{n \cdot}^{-1}\{x\}-1\right)\right) .
$$

Proof. Observe that $\hat{T}^{n} \hat{x}=\hat{x}$ implies $T^{n} \pi_{n}(\hat{x})=\pi_{n}(\hat{x})$ and that $\hat{g}_{n}(\hat{x})=$ $g_{n}\left(\pi_{n}(\hat{x})\right)$.

\section{Examples.}

We evaluate the quotient $\hat{\zeta} / \zeta$ for the examples introduced in $\S 3$.

4.8. Example I (topological Markov shifts, sofic systems, cf. 3.12).

As $\left(\Omega_{A}, S, \mathscr{Z}\right)$ is its own canonical Markov extension, $\hat{\zeta}_{\hat{g}}(\dot{z})=\zeta_{g}(z)$. We note by the way that if $g \equiv 1$ and $\Sigma$ is finite, then $\zeta_{g}(z)=\operatorname{det}(\operatorname{Id}-z A)^{-1}$, see [Bowen/Lanford, 1970].

The zeta function of a sofic system is rational, and its inverse need not be a polynomial (see [Coven/Paul, 1975]).

4.9. Example II (up-and-down-counter, cf. 3.13).

As $0^{\infty}$ and $1^{\infty}$ are the only points which may occur as noninterior points of some $C \in \mathscr{D}$, we see from Lemma 3.11(b) that card $\pi_{n}^{-1}\{x\}=1$ if $T^{n} x=x$ and $x \neq 0^{\infty}, 1^{\infty}$. From the diagram in 3.13 one infers that $\pi_{n}^{-1}\left\{0^{\infty}\right\}=\varnothing$ and $\pi_{n}^{-1}\left\{1^{\infty}\right\}=\left\{\left(1^{\infty}, E\right)\right\}$ for all $n$. Hence

$$
\frac{\hat{\zeta}_{\hat{g}}(z)}{\zeta_{g}(z)}=\exp \left(-\sum_{n=1}^{\infty} \frac{\left(z \cdot g\left(0^{\infty}\right)\right)^{n}}{n}\right)=1-z \cdot g\left(0^{\infty}\right) .
$$

As in Example I we can give a simpler expression for $\zeta_{g}(z)$ if $g \equiv 1: W_{M}$ is a circular code in the sense of [Keller, 1987]. Hence

$$
\begin{aligned}
\exp \left(\sum_{n=1}^{\infty} \frac{z^{n}}{n} \gamma\left(W_{M}^{\infty}, n\right)\right) & =\left(1-\sum_{n=1}^{\infty} z^{n} \cdot \operatorname{card}\left(W_{M} \cap \Sigma^{n}\right)\right)^{-1} \\
& =\left(1-\sum_{n \in M} z^{2 n}\right)^{-1}
\end{aligned}
$$


(ibid., Lemma $1^{17}$ ) and

$$
\zeta(z)=(1-z)^{-2}\left(1-\sum_{n \in M} z^{2 n}\right)^{-1}
$$

(ibid., Lemma 2). In the particular case $M=\mathbb{N}$ we thus have

$$
\zeta(z)=\frac{(1+z)}{(1-z)\left(1-2 z^{2}\right)} .
$$

One should note that, although $\zeta(z)$ is meromorphic, neither $\zeta$ nor $\zeta^{-1}$ can be analytically extended to $\{|z|<r\}$ for $r>1$ in contrast to the situation in Example I. If $M$ is finite, then $\zeta(z)=1 / \operatorname{polynomial}(z)$. (This is not necessarily true for general sofic systems, see e.g. the "even" system of [Weiss, 1973].

4.10. Example III (piecewise monotonic transformations, cf. 3.15).

Suppose that $\tilde{\mathscr{Z}}$ is finite. If $\tilde{x} \in \tilde{X}$ is regular ${ }^{18}$ then $\Phi_{0, \infty}^{-1}\{\tilde{x}\}$ contains exactly one element, and $T^{n} x=x$ if and only if $\tilde{T}^{n} \tilde{x}=\tilde{x}$. On the other hand, if $\tilde{x}$ is not regular, then $\tilde{T}^{k} \tilde{x} \in \operatorname{bd}(Z)$ for some $k \in \mathbb{N}$ and $Z \in \mathscr{Z}$, and there are at most finitely many nonregular points periodic under $\tilde{T}$, each of which has at most two preimages under $\Phi_{0, \infty}$. Hence

$$
\zeta_{g}(z)=\tilde{\zeta}_{\tilde{g}}(z) \tilde{H}(z)
$$

where $\tilde{H}(z)$ is a finite product of expressions of the type

$$
\exp \left( \pm \sum_{n=1}^{\infty} \frac{z^{k n}}{n}\left(g_{k}(x)\right)^{n}\right)=1-z^{k} g_{k}(x)
$$

with $k \in \mathbb{N}$ and $x \in \operatorname{bd}(Z), Z \in \tilde{Z}$. (We have tacitly assumed that $\tilde{g}: \tilde{X} \rightarrow \mathbb{C}$ is continuous on each $Z \in \tilde{\mathscr{Z}}$ such that it can be lifted by $\Phi_{0, \infty}$ to a continuous weight function $g=\tilde{g} \circ \Phi_{0, \infty}$ on $X$.)

Similarly, if $T^{n} x=x$ and if $x$ is interior to each $D \in \mathscr{D}$ it belongs to, then there is a unique $\hat{x} \in \pi_{n}^{-1}\{x\}$ with $\hat{T}^{n} \hat{x}=\hat{x}$ (see Lemma 3.11(b)). But if $x \in \operatorname{bd}(D)$ for some $D=T^{k} Z, Z \in \mathscr{Z}_{k}$ (see Lemma 3.6), then $x \in T^{j}(\operatorname{bd}(Z)$ ) for some $Z \in \mathscr{Z}$ and $0 \leq j \leq k$, i.e. one of the finitely many boundary points of the sets $Z \in \mathscr{Z}$ is ultimately periodic and its orbit contains $x$. Hence $\hat{x} \in \pi_{n}^{-1}\{x\}$ is unique for all but finitely many $x$ from above, and for these exceptional $x$ [Hofbauer, 1986] has shown that there are at most four $\hat{T}$ periodic $\hat{x} \in \pi^{-1}\{x\}$, each of prime period $p$ or $2 p$ if $p$ is the prime period of $x$. Therefore

$$
\zeta_{g}(z)=\hat{\zeta}_{\hat{g}}(z) \hat{H}(z)
$$

17 The mathematical essence of this lemma is similar to Corollary 1 of Theorem 1 in [Takahashi, 1983]. The language used there is very different, however.

${ }^{18}$ I.e. if $\tilde{T}^{k} \tilde{x} \in$ int $V_{n}\left(\tilde{T}^{k} \tilde{x}\right)$ for all $k, n \geq 0$. 
where $\hat{H}(z)$ is again a finite product of expressions of the type (4.15). It will turn out later that the factors $\tilde{H}(z)$ and $\hat{H}(z)$ are unessential for our purposes.

A rather advanced treatment of the continued fraction transformation can be found in [Mayer, 1976]. There it is shown that $\zeta_{g}(z)$ is meromorphic in $\mathbb{C}$ for a wide class of functions $g$ and that $P_{g}$ acts as a nuclear operator on a suitable space of holomorphic functions. The proof relies heavily on the fact that each monotonicity interval is mapped by this transformation onto the whole unit interval.

\section{ANALYTIC STRUCTURES}

The phase space $X$ of a piecewise compact system is in general highly discontinuous; indeed, if the system generates, it is completely disconnected, because each point $x$ possesses a base of closed open neighbourhoods $V_{n}(x)$. Therefore we cannot expect $X$ to carry a differentiable structure in a rigorous sense. On the other hand, if we are primarily interested in interval maps e.g., we have a clear idea of what differentiability of $T$ means, although $(X, T, \mathscr{Z})$ is the system obtained from the "smooth" system $(\tilde{X}, \tilde{T}, \tilde{Z})$ by doubling all preimages of critical points (cf. 3.14-3.15): We simply forget about the doubling (that was necessary in order to avoid troubles in constructing the Markov extension) and re-identify the split up points. In this way some subspace of $\mathrm{mb}(X)$ can be identified with a space of holomorphic functions on a complex neighbourhood of $[0,1]$, and the transfer operator behaves as if it were acting on this space of holomorphic functions. From here it is only a small step to apply such an identification-procedure to systems which we never thought of being smooth, e.g. to subshifts.

We start recalling some facts about spaces of bounded holomorphic functions

\section{A. Bounded holomorphic functions.}

Suppose that $\Omega$ is a region in $\mathbb{C}^{d}$. By $\mathscr{H}^{\infty}(\Omega)$ we denote the space of bounded holomorphic functions on $\Omega$. Endowed with the supremum-norm $\|\cdot\|_{\infty}, \mathscr{H}^{\infty}(\Omega)$ is a Banach space.

The following important theorem will turn out to be responsible for the nuclearity of certain "partial" transfer operators on $\mathscr{H}^{\infty}(\Omega)$ (cf. Theorem 5.8):

5.1. Theorem (of Montel as extended by Grothendieck ${ }^{19}$ ). Let $\Omega_{1}, \Omega_{2}$ be regions in $\mathbb{C}^{d}, \operatorname{cl}\left(\Omega_{1}\right) \subseteq \Omega_{2}$. Then the natural embedding $\mathscr{H}^{\infty}\left(\Omega_{2}\right) \hookrightarrow \mathscr{H}^{\infty}\left(\Omega_{1}\right)$ is not only compact (Montel) but also nuclear (Grothendieck).

\section{B. Piecewise analytic systems.}

Suppose $(X, T, \mathscr{Z}, g)$ is a weighted system and $(\mathscr{D}, \rightarrow)$ is its Markov diagram.

5.2. Definition. A pair $\mathscr{A}=\left(s,\left(U_{D}\right)_{D \in \mathscr{Z}}\right)$ is an analytic structure for $(X, T, \mathscr{Z}, g)$ if there is $d \geq 1$ with:

\footnotetext{
${ }^{19}$ [Grothendieck, 1955], $\S 1$, no. 3
} 
(i) $U_{D}(D \in \mathscr{D})$ are regions in $\mathbb{C}^{d}$, and

$$
\sup \left\{\operatorname{diam}\left(U_{D}\right): D \in \mathscr{D}, U_{D} \neq \mathbb{C}^{d}\right\}<\infty \text {. }
$$

(ii) $s$ is a map from $X$ to $\mathbb{C}^{d}$ with $\operatorname{cl}(s(D)) \subseteq U_{D} \cap \mathbb{R}^{d}$, and $s(D)$ is a uniqueness set for bounded holomorphic functions on $U_{D}$.

(iii) for $C, D \in \mathscr{D}$ with $C \rightarrow D$ there is a holomorphic map $\tau_{C D}: U_{D} \rightarrow U_{C}$ such that $s \circ\left(T_{Z}^{-1}\right)_{\mid D}=\tau_{C D} \circ s_{\mid D}$ if $C \subseteq Z$.

Denote by $D \tau_{C D}$ the total derivative of $\tau_{C D}$ and by $\left\|D \tau_{C D}\right\|$ its Euclidean operator norm on $\mathbb{C}^{d}$. Since $\tau_{C D}\left(U_{D} \cap \mathbb{R}^{d}\right) \subseteq U_{C} \cap \mathbb{R}^{d}, D \tau_{C D}$ has real entries.

(iv) For each $D \in \mathscr{D}$ either $g_{D} \equiv 0$, or there is $\tilde{\psi}_{D} \in \mathscr{H}^{\infty}\left(U_{D}\right)$ with $g_{D}=\exp \left(\tilde{\psi}_{D}\right) \circ s_{\mid D}=: \tilde{g}_{D} \circ s_{\mid D}$. The $\tilde{\psi}_{D}$ and their derivatives are uniformly bounded. Here $g_{D}$ stands for $g_{Z \mid D}$ when $C \subseteq Z$.

For a path $\omega=D_{0} \cdots D_{n}$ let

$$
\tau_{\omega}=\tau_{D_{0}, D_{1}} \circ \cdots \circ \tau_{D_{n-1}, D_{n}}, \quad U_{\omega}=U_{D_{n}}
$$

and

$$
T_{\omega}^{-1}=\left(T_{D_{0}}^{-1} \circ \cdots \circ T_{D_{n-1}}^{-1}\right)_{\mid D_{n}} .
$$

Then $T_{\omega}^{-1}=\left(T_{\mid Z(\omega)}^{n}\right)^{-1}$ (cf. Lemma 3.6) and

$$
s \circ T_{\omega}^{-1}=\tau_{\omega} \circ s_{\mid D_{n}} \text {. }
$$

The meaning of $D \tau_{\omega}$ and $\left\|D \tau_{\omega}\right\|$ is obvious.

\subsection{Definition. $(X, T, \mathscr{Z}, g)$ is $\mathscr{A}$-expanding if}

(i) there are $n_{0} \in \mathbb{N}$ and $\alpha<1$ such that $\left\|D \tau_{\omega}\right\| \leq \alpha$ on $U_{\omega}$ for all paths $\omega$ in $\mathscr{D}$ of length $n \geq n_{0}$, for which $g_{n \mid Z(\omega)} \not \equiv 0$.

(ii) $\operatorname{cl}\left(\tau_{C D}\left(U_{D}\right)\right) \subseteq U_{C}$ for all $C, D \in \mathscr{D}$ with $C \rightarrow D$ and $g_{D} \not \equiv 0$.

5.4. Remark. At first sight it might look sufficient to define analytic structures only on the cylinders $Z \in \mathscr{Z}$ and not on all components $D$ of the Markov extension. However, the notion of an $\mathscr{A}$-expanding system would become more complicated in this case.

\section{Piecewise analytic Markov systems.}

Throughout this subsection suppose that $(X, T, \mathscr{Z}, g)$ is Markovian. Remember that $\mathscr{Z}=\mathscr{D}$ in this case.

5.5. Remark. If $(X, T, \mathscr{Z}, g)$ is $\mathscr{A}$-expanding and if $\omega$ is a closed path of length $n$ in $\mathscr{D}$ such that $g_{n \mid Z(\omega)} \not \equiv 0$, then $\tau_{\omega}$ has a unique fix-point $x_{\omega}$ and $\left\|D \tau_{\omega}\left(x_{\omega}\right)\right\| \leq \alpha^{n / n_{0}}$ (see Lemma 1 of [Ruelle, 1976]). We denote

$$
\kappa(n)=\sum_{\omega}\left\|D \tau_{\omega}\left(x_{\omega}\right)\right\| \cdot\left|g_{n}(\omega)\right|
$$

where the sum extends over all closed paths $\omega$ of length $n$ with $g_{n \mid Z(\omega)} \not \equiv 0$, and

$$
\kappa:=\varlimsup_{n \rightarrow \infty} \kappa(n)^{1 / n} \leq \alpha^{1 / n_{0}} \gamma(\mathscr{Z})
$$


Observe that $\kappa<\gamma(\mathscr{Z})$ if $\gamma(\mathscr{Z})>0$.

5.6. Definition. For an analytic structure $\mathscr{A}$ of $(X, T, \mathscr{Z}, g)$ let $\mathscr{H}^{\infty}(\mathscr{A})=\left\{f \in \operatorname{mb}(X): \forall D \in \mathscr{D} \exists \tilde{f}_{D} \in \mathscr{H}^{\infty}\left(U_{D}\right)\right.$ such that $f_{D}=\tilde{f}_{D} \circ s_{\mid D}$ and $\left.\|f\|_{\mathscr{A}}:=\sum_{D \in \mathscr{D}}\left\|\tilde{f}_{D}\right\|_{\infty}<\infty\right\}$

be the space of $\mathscr{A}$-holomorphic functions on $X$.

Notation: $\left\|f_{D}\right\|_{\mathscr{A}}:=\left\|\tilde{f}_{D}\right\|_{\infty}$.

5.7. Remark. $\left(\mathscr{H}^{\infty}(\mathscr{A}),\|\| \|_{\mathscr{A}}\right)$ is a Banach space. (Observe that the map $\tilde{f}_{D} \mapsto \tilde{f}_{D} \circ s_{\mid D}$ is $1-1$ by Definition 5.2(ii).)

5.8. Theorem. Suppose that $(X, T, \mathscr{Z}, g)$ is $\mathscr{A}$-expanding. For a path $\omega=$ $D_{0} \cdots D_{n}$ define $L_{\omega}: \mathscr{H}^{\infty}(\mathscr{A}) \rightarrow \mathscr{H}^{\infty}(\mathscr{A})$ by

$$
\left(L_{\omega} f\right)(x)= \begin{cases}\left(g_{n} \cdot f\right) \circ T_{\omega}^{-1}(x), & \text { if } x \in D_{n}, \\ 0, & \text { otherwise. }\end{cases}
$$

Then $L_{\omega}$ is nuclear (in the sense of $\S 2 . \mathrm{C}$ ), and

$$
\operatorname{tr}\left(L_{\omega}\right)=g_{n}\left(x_{\omega}\right) \cdot \operatorname{det}\left(\operatorname{Id}-D \tau_{\omega}\left(x_{\omega}\right)\right)^{-1} \text { if } \omega \text { is closed, }
$$

$$
\operatorname{tr}\left(L_{\omega}\right)=0 \text { otherwise, i.e. if } D_{0} \neq D_{n} .
$$

Proof. The nuclearity is an immediate consequence of 5.1. (5:3) is contained in the proof of Lemma 2 of [Ruelle, 1976], and (5.4) follows from Chapter II.3, Proposition 2 of [Grothendieck, 1956].

D. Examples.

5.9. Examples I, II (closed subshifts, cf. 3.12-3.13).

For $j=1, \ldots, d$ let $\phi_{j}: \Sigma \rightarrow \mathbb{C}$ be nonconstant bounded maps, let $\rho_{j} \in \mathbb{C}$, $\left|\rho_{j}\right| \leq \rho<1$, and let $R=\max \left\{\left|\phi_{j}(\sigma)\right| /\left(1-\left|\rho_{j}\right|\right): j=1, \ldots, d ; \sigma \in \Sigma\right\}$. Define $s: \Omega \rightarrow U:=\left\{z \in \mathbb{C}^{d}:\left|z_{j}\right|<R \quad(j=1, \ldots, d)\right\}$ by

$$
s_{j}\left(\sigma_{1}, \sigma_{2}, \sigma_{3}, \ldots\right)=\sum_{i=1}^{\infty} \phi_{j}\left(\sigma_{i}\right) \cdot \rho_{j}^{i} .
$$

For $D \in \mathscr{D}$ let $U_{D}=U$, and if $C \rightarrow D$ in $\mathscr{D}$ then define $\tau_{C D}: U \rightarrow U$ by

$$
\left(\tau_{C D}(z)\right)_{j}=\left(\phi_{j}(\sigma)+z_{j}\right) \cdot \rho_{j} \quad \text { if } C \subseteq[\sigma]_{1} .
$$

We check that $\left(s,\left(U_{D}\right)_{D \in \mathscr{Z}}\right)$ is an analytic structure:

(a) $\left|s_{j}(w)\right| \leq \rho R(w \in \Omega)$, hence $\operatorname{cl}(s(D)) \subseteq U_{D} \quad(D \in \mathscr{D})$.

(b) Let $C \subseteq[\sigma]_{1}, C \rightarrow D, w \in D$. Then

$$
\left(s\left(T_{C}^{-1} w\right)\right)_{j}=s_{j}(\sigma w)=\rho_{j}\left(\phi_{j}(\sigma)+s_{j}(w)\right)=\left(\tau_{C D}(s(w))\right)_{j},
$$

i.e. $s \circ\left(T_{C}^{-1}\right)_{\mid D}=\tau_{C D} \circ S_{\mid D}$. 
(c) For $d=1$ it is an easy excercise to show that $s(D)$ is a uniqueness set $(D \in \mathscr{D})$ in case of Examples I and II, if one assumes for Example I that there are no trivial cylinders (i.e. consisting of finitely many points only). The corresponding assertion for $d>1$ follows, because now $s(D)$ is the Cartesian product of one-dimensional uniqueness sets.

(d) Obviously the $\tau_{C D}$ are holomorphic on $U$ and $\left\|D \tau_{C D}\right\|=\prod_{j=1}^{d} \rho_{j} \leq$ $\rho^{d}<1$. Finally, for $z \in U$

$$
\left|\left(\tau_{C D}(z)\right)_{j}\right| \leq \rho(R(1-\rho)+R)=\rho(2-\rho) R<R
$$

such that $\operatorname{cl}\left(\tau_{C D} U\right) \subseteq U$ and $(\Omega, S, \mathscr{D})$ is $\mathscr{A}$-analytic. In particular $\kappa \leq$ $\gamma(\mathscr{D}) \prod_{j=1}^{d} \rho_{j}$.

Similarly one may choose $U_{D}=\mathbb{C}(D \in \mathscr{D})$ and $\tau_{C D}: \mathbb{C} \rightarrow \mathbb{C}, z \mapsto 0$ if $C \rightarrow D$. In this case $\kappa=0$.

5.10. Example III (piecewise monotonic transformations, cf. 3.14-3.15).

Let $s=\Phi_{0, \infty}$, and for $D \in \mathscr{D}$ and $\varepsilon>0$ let $U_{D}=\{z \in \mathbb{C}$ : $\operatorname{dist}(z, s(D))<\varepsilon\}$. $U_{D}$ is a region in $\mathbb{C}$ and $\operatorname{cl}(s(D)) \subseteq U_{D}$. Suppose that $\left(\tilde{T}_{\tilde{Z}}^{-1}\right)_{\mid \tilde{T} \tilde{Z}}$ has a bounded holomorphic extension to a complex neighbourhood of $\tilde{T} \tilde{Z}(\tilde{Z} \in \tilde{Z})$ and that $\inf _{\tilde{x} \in \dot{X}}\left|\tilde{T}^{\prime}(\tilde{x})\right|>1$. Now, if $\tilde{\mathscr{Z}}$ is finite, $\varepsilon>0$ can be chosen small enough such that there exist extensions $\tau_{C D}$ of $\left(\tilde{T}_{s(Z)}\right)_{\mid s(D)}^{-1}$ to $U_{D}$ (if $C \rightarrow D$ and $C \subseteq Z$ ), and by choosing $\varepsilon$ even smaller (if necessary), one may assume

$$
\left|\tau_{C D}^{\prime}\right| \leq \alpha<1 \quad \text { for some } \alpha<1 .
$$

In particular $\operatorname{cl}\left(\tau_{C D}\left(U_{D}\right)\right) \subseteq U_{C}$ for such $C, D$.

We still have to assure that $s(D)$ is a uniqueness set for bounded holomorphic functions on $U_{D},(D \in \mathscr{D})$, i.e. $\operatorname{card}(s(D))=\infty$ for all $D \in \mathscr{D}$. This requirement is fullfilled, however, since it is easily seen that the endpoints of each $D \in \mathscr{D}$ are non-isolated in $D$. Hence $\mathscr{A}=\left(s,\left(U_{D}\right)_{D \in \mathscr{C}}\right)$ is an analytic structure for $(X, T, \mathscr{Z}, g)$ for suitable $g$, e.g. for $g=1 /\left|\tilde{T}^{\prime} \circ s\right|$, (just the structure on $X$ inherited from $\tilde{X} \subseteq \mathbb{R})$, and $(X, T, \mathscr{Z}, g)$ is $\mathscr{A}$-expanding.

\section{SPectral theORY FOR MARKov systems}

\section{A. The main theorems.}

Assume throughout this section that $(X, T, \mathscr{Z}, g)$ is a weighted Markov system, expanding with respect to the analytic structure $\mathscr{A}=\left(s,\left(U_{D}\right)_{D \in \mathscr{U}}\right)$.

Fix an increasing sequence $\left(\mathscr{E}_{n}\right)_{n \in N}$ of finite subsets of $\mathscr{D}$ such that $\bigcup_{n \in \mathrm{N}} \mathscr{E}_{n}=\mathscr{D}$, and define projections $\beta\left[\mathscr{E}_{n}\right]: \mathscr{H}^{\infty}(\mathscr{A}) \rightarrow \mathscr{H}^{\infty}(\mathscr{A})$ by

$$
(\beta[\mathscr{E}](f))_{Z}= \begin{cases}f_{Z} & \text { if } Z \in \mathscr{E}, \\ 0 & \text { otherwise, }\end{cases}
$$

$\mathscr{F}=\left\{\beta\left[\mathscr{E}_{n}\right]: n \in \mathbb{N}\right\}$ is a continuous projection net (see Definition 2.1).

Finally suppose that $\phi \in \mathrm{mb}(X)$ is real-valued, $\phi_{D}=\tilde{\phi}_{D} \circ s$ for some $\tilde{\phi}_{D} \in$ $\mathscr{H}^{\infty}\left(U_{D}\right)(D \in \mathscr{D})$, and that the $\tilde{\phi}_{D}$ are uniformly bounded. 
Under these assumptions and with the notational conventions of $\S 2$.D we can state

6.1. Theorem. Suppose that the Markov diagram of $(X, T, \mathscr{Z})$ is uniformly forward finite. ${ }^{20}$

(a) The transfer operator $P=P_{g \cdot \exp (i \phi)}$ leaves $\mathscr{H}^{\infty}(\mathscr{A})$ invariant.

(b) If $r_{\text {as }}<r(P)$, then $P$ is quasicompact on $\mathscr{H}^{\infty}(\mathscr{A})$ and $r_{\text {ess }} \leq r_{\text {as }}$. If $\phi \equiv 0$ and $g \geq 0$, then $r_{\text {ess }}=r_{\text {as }}$.

(c) If $r_{\text {as }}<r(P)$, if there is some Borel measure $m$ on $X$ with $\operatorname{supp}(m)=X$, $\sup _{D \in \mathscr{D}} m(D)<\infty$, and such that $g^{-1}$ is the derivative of $T$ with respect to $m,{ }^{21}$ and if $\mathscr{H}^{\infty}(\mathscr{A})$ is dense in $L_{m}^{1}$, then $P$ admits a representation

$$
P=\sum_{i=1}^{l} \lambda_{i} E_{i}+\sum_{i=l+1}^{N} \lambda_{i}\left(E_{i}+N_{i}\right)+Q
$$

with $E_{i}, N_{i}$, and $Q$ as in (2.2) and where $\Gamma=\left\{\lambda_{1}, \ldots, \lambda_{l}\right\} \neq \varnothing$ is the set of eigenvalues of modulus 1 , and $\left|\lambda_{j}\right|<1$ for $j=l+1, \ldots, N$. For each $\lambda_{i} \in \Gamma$

$$
E_{i}=\lim _{n \rightarrow \infty} \frac{1}{n} \sum_{k=0}^{n-1}\left(\lambda_{i}^{-1} P\right)^{k}
$$

uniformly on the unit ball of $\mathscr{H}^{\infty}(\mathscr{A}) . E_{i}$ extends to $L_{m}^{1},\left\|E_{i}\right\|_{1}=1$, and (6.2) holds strongly on $L_{m}^{1}$. $\left(P-\sum_{i=1}^{l} \lambda_{i} E_{i}\right)^{n}$ tends to 0 as $n \rightarrow \infty$ strongly on $L_{m}^{1}$. If $P f=\lambda f$ for some $0 \neq f \in L_{m}^{1}$ and $|\lambda|=1$, then $f \in \mathscr{H}^{\infty}(\mathscr{A})$ and $\lambda \in \Gamma$.

(If $\operatorname{supp}(m) \neq X$, then (6.1) and (6.2) still hold in the $L_{m}^{1}$-sense.)

(d) If, in the situation of (c), $\phi \equiv 0$, i.e. if $P$ is the classical Perron-Frobenius operator satisfying (4.3), then $\lambda_{1}=1 \in \Gamma, E_{1}$ is positive, and $\Gamma$ is fully cyclic. ${ }^{22}$

Let $d:=\operatorname{rank}\left(E_{1}\right)$. There are a mod m-partition $\left(X_{1}, \ldots, X_{d}\right)$ of $X$ into $T-T^{-1}$-invariant measurable sets and a basis $\left\{h_{1}, \ldots, h_{d}\right\}$ of $\operatorname{range}\left(E_{1}\right)$ such that $h_{i} \geq 0$ and $\int_{X_{i}} h_{j} d m=\delta_{i j}(i, j=1, \ldots, d)$. If $h$ is any convex combination of the $h_{i}$ and $d \mu=h d m$, then $\mu$ is a T-invariant probability measure.

(e) If $P_{g}$ satisfies the assumptions of (c) and (d) and if the eigenspace range $\left(E_{1}\right)$ of $P_{g}$ is one-dimensional, then (without any further assumptions on $\left.P=P_{g \cdot \exp (i \phi)}\right) P: L_{m}^{1} \rightarrow L_{m}^{1}$ has an eigenvalue of modulus 1 if and only if $\exp (i \phi)$ is equivalent to a constant, i.e. there are $a \in \mathbb{R}$ and $\psi \in \operatorname{mb}(X)$ such that $\exp (i \phi)=\exp i(a+\psi \circ T-\psi)$ m-a.e.

6.2. Theorem. Suppose $(X, T, \mathscr{Z})$ generates, that the Markov diagram of $(X, T, \mathscr{Z}, g)$ is uniformly forward finite and that $g \geq 0$. Let $P=P_{g}$ and

$$
\gamma_{\text {as }}=\lim _{n \rightarrow \infty} \gamma\left(\mathscr{D} \backslash \mathscr{E}_{n}\right) \quad(c f .(4.7)) \text {. }
$$

\footnotetext{
${ }^{20}$ See Definition 3.7.

${ }^{21}$ Cf. Remark 4.3.

22 I.e. $P f=\lambda f,|\lambda|=1$, implies $P\left(f^{k} /|f|^{k-1}\right)=\lambda^{k} f^{k} /|f|^{k-1}$ for all $k \in \mathbf{Z}$, see Definition V.4.5 in [Schaefer, 1974].
} 
Assume

(i) $r(P)<\infty, \quad \gamma(\mathscr{D})<\infty$,

(ii) $\left[\gamma_{\text {as }}<\gamma(\mathscr{D})\right.$ or $\left.\gamma_{\text {as }} \leq r(P)\right]$ and $\left[r_{\text {as }}<r(P)\right.$ or $\left.r_{\text {as }} \leq \gamma(\mathscr{D})\right]$,

(iii) $\lim _{\alpha \in \mathscr{F}}\left\|\alpha\left(P_{\beta} \alpha\right)^{j} P \beta-P_{\beta}^{j} P \beta\right\|_{T}=0$ for all $\beta \in \mathscr{F}$ and $j \in \mathbb{N} .^{23}$

Then $P: \mathscr{H}^{\infty}(\mathscr{A}) \rightarrow \mathscr{H}^{\infty}(\mathscr{A})$ is $\mathscr{F}$-quasinuclear and $r(P)=\gamma(\mathscr{D})$. The inverse of the zeta function $\zeta_{g}(z)$ can be analytically extended from $\{|z|<$ $\left.\gamma(\mathscr{D})^{-1}\right\}$ to ${ }^{24}$

$$
G_{\mathscr{F}}:=\left\{z \in \mathbb{C}:|z|<1 / \max \left\{\gamma_{\mathrm{as}}, r_{\mathrm{as}}, \kappa\right\}\right\},
$$

and $\operatorname{det}_{\mathscr{F}}\left(\mathrm{Id}-z P_{g}\right) \cdot \zeta_{g}(z)$ is analytic and nonzero on $G_{\mathscr{F}} \cdot z_{0} \in G_{\mathscr{F}}$ is a pole of $\zeta_{g}(z)$ if and only if $z_{0}^{-1}$ is an eigenvalue of $P_{g}$. The order of the pole $z_{0}$ of $\zeta_{g}(z)$ is finite and equals the algebraic multiplicity of the eigenvalue $z_{0}^{-1}$ of $P_{g}$.

Even without assuming (ii) $1 / \zeta_{g}(z)$ can be analytically extended to $G_{\mathscr{F}}$.

6.3. Remark. The assumption in both theorems that the Markov diagram of $(X, T, \mathscr{Z})$ is uniformly forward finite is needed only for showing that $P$ is a bounded operator on $\mathscr{H}^{\infty}(\mathscr{A})$ and that $P \alpha$ is nuclear for $\alpha \in \mathscr{F}$. It can be replaced by these two assertions. Alternatively one could assume in view of Remark $2.4(\mathrm{c})$ that $P$ is bounded on $\mathscr{H}^{\infty}(\mathscr{A})$ and $\alpha P$ is nuclear for $\alpha \in \mathscr{F}$. In this case the trace-norm in assumption (iii) of Theorem 6.2 may be replaced by the usual operator-norm. With these changes both theorems apply also to systems like the continued fraction transformation, but it may be quite hard to verify these assumptions (see 4.10 and [Mayer, 1976]).

6.4. Remark. Even if $G_{\mathscr{F}}$ is the greatest ball around 0 to which $\zeta_{g}(z)^{-1}$ can be analytically extended, it may still be possible to extend $\zeta_{g}(z)$ meromorphically to a bigger ball (see also 6.13, Example II).

The proof of the theorems needs the following monotonicity lemma:

6.5. Lemma. Assume that $g \geq 0$. Let $\Omega_{1} \subseteq \Omega_{2}$ be sets of paths of length $n$ in $D$, and suppose that there are $f_{\omega} \in \mathscr{H}^{\infty}(\mathscr{A})\left(\omega \in \Omega_{1}\right)$. If $a_{D}(D \in \mathscr{D})$ are positive constants with $\left\|\left(f_{\omega}\right)_{D}\right\|_{\mathscr{A}} \leq a_{D}\left(\omega \in \Omega_{1}\right)$ and if $f^{*}=\sum_{D \in \mathscr{D}} a_{D} \chi_{D}$, then

$$
\sum_{\omega \in \Omega_{1}}\left\|\left(f_{\omega} \cdot g_{n}\right) \circ T_{\omega}^{-1}\right\|_{\mathscr{A}} \leq A \cdot\left\|\sum_{\omega \in \Omega_{2}}\left(f^{*} \cdot g_{n}\right) \circ T_{\omega}^{-1}\right\| \|_{\mathscr{A}}
$$

with a constant $A$ independent of $n, \Omega_{1}, \Omega_{2}, f_{\omega}$, and $f^{*}$.

Proof. Observing that $(X, T, \mathscr{Z}, g)$ is $\mathscr{A}$-expanding and that $\tilde{g}_{D} \equiv 0$ or $\tilde{g}_{D}=\exp \tilde{\psi}_{D} \in \exp \mathscr{H}^{\infty}\left(U_{D}\right)$ for $D \in \mathscr{D}$, where the derivatives of the $\tilde{\psi}_{D}$ are uniformly bounded, it needs a routine calculation to show that there is a constant $B>0$ with

$$
\left|\tilde{g}_{n}\left(\tau_{\omega} z_{1}\right)\right| \leq\left|\tilde{g}_{n}\left(\tau_{\omega} z_{2}\right)\right| \cdot \exp \left(B\left|z_{1}-z_{2}\right|\right)
$$

$23\|.\|_{T}$ is the trace-norm, see $\S 2 . C$.

24 For $\kappa$ see Remark 5.5. 
for $n \in \mathbb{N}$ and $z_{1}, z_{2}$ in the domain of $\tau_{\omega}, \omega=D_{0} \cdots D_{n}$. In particular

$$
\left|\tilde{g}_{n}\left(\tau_{\omega} z_{1}\right)\right| \leq \text { const } \cdot\left|\tilde{g}_{n}\left(\tau_{\omega} z_{2}\right)\right|
$$

for $n, \omega, z_{1}, z_{2}$ as above. If $U_{D_{n}} \neq \mathbb{C}^{d}$ this follows from the uniform boundedness of the sets $U_{D} \neq \mathbb{C}^{d}$ (see Definition 5.2(i)). Otherwise $\tilde{g}$ is constant on $U_{D_{n}}$, and it suffices to treat $\tilde{g}_{n-1} \circ \tau_{\omega^{\prime}}$ where $\omega^{\prime}=D_{0} \cdots D_{n-1}$.

Now the positivity of $g$ implies

$$
\begin{aligned}
& \sum_{\omega \in \Omega_{1}}\left\|\left(f_{\omega} \cdot g_{n}\right) \circ T_{\omega}^{-1}\right\|_{\mathscr{A}} \leq \sum_{\substack{D \in \mathscr{Z} \\
(\in \mathcal{L}}} \sum_{\substack{\left(\omega \in \Omega_{1} \\
(\nu) \cdots \cdots D\right.}}\left\|\left(f_{\omega}\right)_{C}\right\|_{\mathscr{A}} \cdot\left\|\tilde{g}_{n} \circ \tau_{\omega}\right\|_{\infty} \\
& \leq \sum_{\substack{D \in \mathscr{Z} \\
(\in \mathcal{X}}} \sum_{\substack{\omega \in \Omega_{2} \\
(j)(\cdots)}} a_{C} \cdot \text { const } \cdot \min _{x \in D}\left\{\tilde{g}_{n} \circ \tau_{\omega}(s(x))\right\} \\
& \leq \text { const } \cdot \sum_{D \in \mathscr{Z}}\left\|\sum_{\substack{\omega \in \Omega_{2} \\
\omega=\ldots \bar{D}}}\left(f^{*} \cdot g_{n}\right) \circ T_{\omega}^{-1}\right\|_{\mathscr{A}} \\
& \text { = const } \cdot\|\| \sum_{\omega \in \Omega_{2}}\left(f^{*} \cdot g_{n}\right) \circ T_{\omega}^{-1}\|\|_{\mathscr{A}} \text {. }
\end{aligned}
$$

6.6. Remark. The need for such a lemma is the main reason why I restricted Theorem 6.2 to the case $g \geq 0$. If Lemma 6.5 holds because of other reasons, most of the theorem remains true for more general $g$.

Proof of Theorem 6.1. As the Markov diagram of $(X, T, \mathscr{Z})$ is uniformly forward finite, $P \beta(\beta \in \mathscr{F})$ is a finite sum of operators of the type $f \mapsto$ $(f g \exp (i \phi)) \circ T_{C D}^{-1}$ each of which is nuclear by Theorem 5.8. Hence $P \beta$ is nuclear and a fortiori compact for $\beta \in \mathscr{F}$. $P$ leaves $\mathscr{H}^{\infty}(\mathscr{A})$ invariant, because for $f \in \mathscr{H}^{\infty}(\mathscr{A})$

$$
\begin{aligned}
\|P f\|_{\mathscr{A}} & \leq \sum_{D \in \mathscr{Z}} \sum_{\substack{(\in \mathscr{Z}) \\
(\rightarrow D)}}\left\|(g \cdot \exp (i \phi) \cdot f) \circ\left(T_{C}^{-1}\right)_{\mid D}\right\|_{\mathscr{A}} \\
& \leq \sum_{C \in \mathscr{Z}} \sum_{\substack{D \in \mathscr{Z} \\
(\rightarrow D)}}\left\|g_{C} \exp \left(i \phi_{C}\right)\right\|_{\mathscr{A}}\left\|f_{C}\right\|_{\mathscr{A}} \\
& \leq \mathrm{const} \cdot \sup _{C \in \mathscr{Z}}\left\|g_{C} \exp \left(i \phi_{C}\right)\right\|_{\mathscr{A}} \cdot\|f\|_{\mathscr{A}}
\end{aligned}
$$

The quasicompactness of $P$ and $r_{\text {ess }} \leq r_{\text {as }}$ follow from Proposition 2.2.

Suppose now $\phi \equiv 0$ and $g \geq 0$. For $f \in \mathscr{H}^{\infty}(\mathscr{A})$ let $f^{*}=$ $\sum_{D \in \mathscr{Z}}\left\|f_{D}\right\|_{\mathscr{H}} \cdot \chi_{D}$. Then $f^{*} \in \mathscr{H}^{\infty}(\mathscr{A}),\left\|f^{*}\right\|_{\mathscr{A}}=\left\|f_{\|}\right\|_{\mathscr{A}}$, and for $\alpha, \beta \in \mathscr{F}$ with $\alpha \geq \beta$ and $r_{\alpha} \geq r_{\text {as }}-\varepsilon$ :

$$
\begin{aligned}
\|f \mid\|_{. \mathscr{V}} & \cdot\left\|(\mathrm{Id}-\beta) P^{n}(\mathrm{Id}-\beta)\right\|_{\mathscr{A}} \\
& \geq\|\|(\mathrm{Id}-\beta) P^{n}(\mathrm{Id}-\beta) f^{*} \|_{\mathscr{S}} \\
& \geq A^{-1} \cdot\left\|P_{\alpha}^{n} f\right\|_{\mathscr{S}} \quad \text { (by Lemma 6.5). }
\end{aligned}
$$


Hence

$$
A \cdot\left\|(\operatorname{Id}-\beta) P^{n}(\mathrm{Id}-\beta)\right\|_{\mathscr{A}} \geq \mid\left\|P_{\alpha}^{n}\right\|_{\mathscr{A}} \geq r_{\alpha}^{n} \geq\left(r_{\text {as }}-\varepsilon\right)^{n},
$$

and $r_{\text {ess }}=r_{\text {as }}$ follows from Proposition 2.2. This proves (a) and (b).

Suppose now that there is a measure $m$ on $X$ with $\operatorname{supp}(m)=X$ and with the properties from Remark 4.3. If $\left|\lambda_{i}\right| \geq 1$ and if $P f=\lambda_{i} f$ for some $0 \neq f \in \mathscr{H}^{\infty}(\mathscr{A})$, then $\left|\lambda_{i}\right||f|=|P f| \leq P_{g}|f|$ and $0<\int|f| d m=\int P_{g}|f| d m$, whence $\left|\lambda_{i}\right|=1$. We show that $N_{i} \equiv 0:$ Suppose there is $f \in E_{i}\left(\mathscr{H}^{\infty}(\mathscr{A})\right)$ with $N_{i} f \neq 0$ (i.e. $\int\left|N_{i} f\right| d m>0$ as $\operatorname{supp}(m)=X$ ) and $N_{i}^{2} f=0$. Then by (2.2) and (4.5)

$$
0<\int\left|N_{i} f\right| d m \leq \lim _{n \rightarrow \infty} \frac{1}{n}\left(\int P_{g}^{n}|f| d m+\int\left|E_{i} f\right| d m\right) \leq 0,
$$

a contradiction. Hence $N_{i} \equiv 0$ if $\left|\lambda_{i}\right|=1$. This yields the representation (6.1). (If $\operatorname{supp}(m) \neq X$, practically the same argument shows that (6.1) still holds in the $L_{m}^{1}$-sense.)

For $|\lambda|=1$ and $n \in \mathbb{N}$ let $S_{\lambda, n}=\frac{1}{n} \sum_{k=0}^{n-1}\left(\lambda^{-1} P\right)^{k}$. Because of (6.1) $\lim _{n \rightarrow \infty} S_{\lambda, n}=E_{i}$ if $\lambda=\lambda_{i} \in \Gamma$ and $\lim _{n \rightarrow \infty} S_{\lambda, n}=0$ if $\lambda \notin \Gamma$ (uniformly on bounded subsets of $\left.\mathscr{H}^{\infty}(\mathscr{A})\right)$. As $\int|P f| d m \leq \int P_{g}|f| d m=\int|f| d m, P$ and also $S_{\lambda, n}$ are $L_{m}^{1}$-contractions, and since $\mathscr{H}^{\infty}(\mathscr{A})$ is dense in $L_{m}^{1}, S_{\lambda, n}$ converges strongly on $L_{m}^{1}$ thereby extending $E_{i}$ to a projection from $L_{m}^{1}$ onto $E_{i}\left(\mathscr{H}^{\infty}(\mathscr{A})\right)$ with $\left\|E_{i}\right\|_{1}=1$. (The strong convergence to 0 as $n \rightarrow \infty$ of $\left(P-\sum_{i=1}^{l} \lambda_{i} E_{i}\right)^{n}$ is an immediate consequence.) For $0 \neq f \in L_{m}^{1}$ and $|\lambda|=1$ with $P f=\lambda f$ we thus obtain $0 \neq f=\lim _{n \rightarrow \infty} S_{\lambda, n} f=E_{i} f \in \mathscr{H}^{\infty}(\mathscr{A})$ and $\lambda=\lambda_{i} \in \Gamma$. This finishes the proof of (c) and we turn to (d):

Let $\phi \equiv 0$. For $\lambda=1$ and $f \in L_{m}^{1}$ with $\int f d m \neq 0$ identity (4.3) implies

$$
0 \neq \int f d m=\lim _{n \rightarrow \infty} \int S_{1, n} f d m=\int E_{1} f d m
$$

and hence $1=\lambda_{1} \in \Gamma$. Obviously $E_{1}$ is positive in this case. $\Gamma$ is fully cyclic by Proposition V.4.6 in [Schaefer, 1974] and the example thereafter. As $f \in \operatorname{range}\left(E_{1}\right)$ implies $\bar{f},|f| \in \operatorname{range}\left(E_{1}\right),{ }^{25} \operatorname{range}\left(E_{1}\right)$ is a vector sublattice of $L_{m}^{1}$ (Riesz subspace).

Hence there are $0 \leq h_{i} \in \operatorname{range}\left(E_{1}\right), \int h_{i} d m=1(i=1, \ldots, d)$ with mutually disjoint supports $H_{i} \cdot{ }^{26}$ As

$$
\int_{T^{-n} H_{l}} h_{j} d m=\int_{H_{l}} P^{n} h_{j} d m=\int_{H_{i}} h_{j} d m=\delta_{i j},
$$

we have for $X_{i}:=\bigcup_{n \geq 0} T^{-n} H_{i}$

$$
\int_{X_{i}} h_{j} d m=\delta_{i j}
$$

${ }^{25}$ The latter because $P f=f \Rightarrow|f| \leq P|f|$ and $\int|f| d m=\int P|f| d m$.

${ }^{26}$ See the preliminaries of $\S 1$ in [Schaefer, 1980]. 
In view of Remark 4.3 the assumption $\operatorname{supp}(m)=X$ implies $g>0$. Therefore $T H_{i}=H_{i}$ and the $X_{i}$ are disjoint and $T-T^{-1}$-invariant. Let $B=X \backslash \bigcup_{i=1}^{d} X_{i}$. Then

$$
\begin{aligned}
m(B) & =\int_{X} E_{1} \chi_{B} d m=\sum_{i=1}^{d} \int_{X_{i}} E_{1} \chi_{B} d m \\
& =\lim _{n \rightarrow \infty} \sum_{i=1}^{d} \frac{1}{n} \sum_{k=0}^{n-1} \int_{X_{i}} P^{k} \chi_{B} d m \\
& =0,
\end{aligned}
$$

because $\int_{X_{i}} P^{k} \chi_{B} d m=\int_{T^{-k} X_{i}} \chi_{B} d m=0$, i.e. $\left(X_{1}, \ldots, X_{d}\right)$ is a partition of $X$ modulo $m$-null sets.

As the $T$-invariance of the measure $d \mu=h d m$ follows from (4.4), this finishes the proof of $(d)$.

We prove (e): Let $d=1, h=h_{1}$. Suppose there are $a \in \mathbb{R}$ and $\psi \in \operatorname{mb}(X)$ such that

$$
\exp (i \phi)=\exp i(a+\psi \circ T-\psi) \quad m \text {-a.e. }
$$

Then

$$
\begin{aligned}
P(h \cdot \exp (i \psi)) & =P_{g}(h \cdot \exp i(\phi+\psi)) \\
& =\exp (i a) P_{g}(h \cdot \exp (i \psi \circ T)) \\
& =\exp (i a) \cdot h \cdot \exp (i \psi) \quad m \text {-a.e., }
\end{aligned}
$$

i.e. $\exp (i a)$ ia an eigenvalue of $P: L_{m}^{1} \rightarrow L_{m}^{1}$.

Conversely, let $P f=\lambda f$ for $0 \neq f \in L_{m}^{1}$ and $|\lambda|=1$. Assume without loss of generality that $\int|f| d m=1, f=|f| \cdot \exp (i \psi)$, and $\lambda=\exp (i a), a \in \mathbf{R}$. Then

$$
|f| \cdot \exp i(a+\psi)=P f=P_{g}(f \cdot \exp (i \phi)) \quad m \text {-a.e. },
$$

i.e.

$$
|f|=P_{g}(|f| \cdot \exp i(-a+\phi-\psi \circ T+\psi)) \leq P_{g}|f| \quad m \text {-a.e. }
$$

as $P_{g}$ is positive and, because of (4.3) $P_{g}|f|=|f|$, i.e. $|f|=h$, and

$$
\exp i(-a+\phi-\psi \circ T+\psi)=1 \quad m \text {-a.e. }
$$

on $\{h \neq 0\}$. This means that (6.5) holds $\mu$-a.e. or, equivalently, $m$-a.e. on $H=\operatorname{supp}(h)$. Since the identity $f=h \cdot \exp (i \psi)$ determines $\psi(\bmod 2 \pi)$ only on $H$ and since $X=\bigcup_{n \geq 0} T^{-n} H$ modulo $m$, the domain of validity of (6.5) can be iteratively extended to $X(\bmod m)$.

6.7. Remark. Most ideas in the proof of (c)-(e) of Theorem 6.1 have already occured in one or another form in the literature, some of them several times. In a similar situation, [Pollicott, 1984] proved (e). His proof is slightly different. 
Proof of Theorem 6.2. At the beginning of the proof of the last theorem we saw that $P: \mathscr{H}^{\infty}(\mathscr{A}) \rightarrow \mathscr{H}^{\infty}(\mathscr{A})$ is bounded (see (6.4)) and that all $P \beta(\beta \in \mathscr{F})$ are nuclear. Let $\alpha=\beta\left[\mathscr{E}_{k}\right], \beta=\beta\left[\mathscr{E}_{l}\right], k \geq l$. Then

$$
\begin{aligned}
\operatorname{tr}\left(\left(\alpha P_{\beta} \alpha\right)^{n}\right) & =\sum_{\substack{\omega=D_{0} \ldots D_{n} \\
D_{i} \in \sum_{k} \backslash \varepsilon_{i}}} \operatorname{tr}\left(f \mapsto\left(g_{n} \cdot f\right) \circ \tau_{\omega}\right) \\
& =\sum_{\substack{\omega=D_{0} \ldots D_{n-1} \\
D_{i} \in \ell_{k} \backslash Y_{i}}} g_{n}\left(x_{\omega}\right) \cdot \operatorname{det}\left(\operatorname{Id}-D \tau_{\omega}\left(x_{\omega}\right)\right)^{-1}
\end{aligned}
$$

by Theorem 5.8. As $(X, T, \mathscr{Z}, g)$ is $\mathscr{A}$-expanding, $g_{n}\left(x_{\omega}\right)=0$ or $\left\|D \tau_{\omega}\left(x_{\omega}\right)\right\| \leq \alpha^{n / n_{0}}<1$ by Remark 5.5. Therefore, if $d$ is the dimension of the analytic structure $\mathscr{A}$ and if $g_{n}\left(x_{\omega}\right) \neq 0$, then

$$
\left(\frac{1}{1+\left\|D \tau_{\omega}\left(x_{\omega}\right)\right\|}\right)^{d} \leq \operatorname{det}\left(\operatorname{Id}-D \tau_{\omega}\left(x_{\omega}\right)\right)^{-1} \leq\left(\frac{1}{1-\left\|D \tau_{\omega}\left(x_{\omega}\right)\right\|}\right)^{d} .
$$

(Observe that $D \tau_{\omega}\left(x_{\omega}\right)$ has real entries, see Definition 5.2(iii).)

Hence all summands in (6.6) are positive, and the expression is monotone in $k$ if $l$ is fixed. Therefore

$$
\lim _{\alpha \in \mathscr{F}} \operatorname{tr}\left(\left(\alpha P_{\beta} \alpha\right)^{n}\right)=\operatorname{tr}_{\mathscr{F}}\left(P_{\beta}^{n}\right)
$$

exists (possibly $=\infty$ ). Observing the positivity of $g$ we thus obtain from (6.6), (6.7), and (4.6) for $\beta=\beta\left[\mathscr{E}_{l}\right]$ and $\mathscr{A} \subseteq \mathscr{E}_{l} \subseteq \mathscr{B} \subseteq \mathscr{D}$

$$
c_{n} \cdot \gamma(\mathscr{D} \backslash \mathscr{B}, n) \leq \operatorname{tr}_{\mathscr{F}}\left(P_{\beta}^{n}\right) \leq c_{n}^{-1} \cdot \gamma(\mathscr{D} \backslash \mathscr{A}, n)
$$

for some constants $0<c_{n} \nearrow 1$. In particular

$$
t_{\beta}=\varlimsup_{n \rightarrow \infty}\left|\operatorname{tr}_{\mathscr{F}}\left(P_{\beta}^{n}\right)\right|^{1 / n}=\varlimsup_{n \rightarrow \infty}\left|\gamma\left(\mathscr{D} \backslash \mathscr{E}_{l}, n\right)\right|^{1 / n}=\gamma\left(\mathscr{D} \backslash \mathscr{E}_{l}\right)
$$

and

$$
t_{\mathrm{as}}=\lim _{\beta \in \mathscr{F}} t_{\beta}=\lim _{l \rightarrow \infty} \gamma\left(\mathscr{D} \backslash \mathscr{E}_{l}\right)=\gamma_{\mathrm{as}} .
$$

Now $t(P)=t_{0}=\gamma(D)<\infty$ by (6.9), and all further assumptions on $t$ in (ii) and (iii) of Definition 2.3 follow immediately from (6.8) and (6.9).The corresponding assumption on $r$ in (iii) of Definition 2.3 is easily verified using Lemma 6.5 (i.e. the positivity of $P_{g}$ ). Finally, (iv) of Definition 2.3 is nothing but assumption (iii) of the theorem. Hence $P$ is $\mathscr{F}$-quasinuclear.

Now the theorem will follow from Theorem 2.5 and Corollary 2.6, if we can show that $\operatorname{det}_{\mathscr{F}}(\operatorname{Id}-z P) \cdot \zeta(z)$ is analytic and nonzero on $G_{\mathscr{F}}$. (Note that $r(P)$ is then an eigenvalue of $P$, because $r(P)^{-1}=t(P)^{-1}=\gamma(\mathscr{D})^{-1}$ is a pole of $\zeta(z)$ and hence of $\operatorname{det}_{\mathscr{F}}(\operatorname{Id}-z P)$.) 
Observing (2.14) and (4.10) we have

$$
\begin{aligned}
\operatorname{det}_{\mathscr{F}}(\operatorname{Id}-z P) \cdot \zeta(z) & =\exp \left(\sum_{n=1}^{\infty} \frac{z^{n}}{n}\left(-\operatorname{tr}_{\mathscr{F}}\left(P^{n}\right)+\gamma(\mathscr{D}, n)\right)\right) \\
& =\exp \left(\sum_{n=1}^{\infty} \frac{z^{n}}{n} \sum_{\substack{\text { colosed path of } \\
\text { length } n \text { in } \mathscr{Y}}} g_{n}\left(x_{\omega}\right)\left(1-\operatorname{det}\left(\operatorname{Id}-D \tau_{\omega}\left(x_{\omega}\right)\right)^{-1}\right)\right),
\end{aligned}
$$

and by (6.7) $\operatorname{det}_{\mathscr{F}}(\mathrm{Id}-z P) \zeta(z)$ is analytic and nonzero for

$$
\begin{aligned}
|z| & \leq 1 / \varlimsup_{n \rightarrow \infty}\left(\sum_{\omega} g_{n}\left(x_{\omega}\right)\left(\left(\frac{1}{1-\left\|D \tau_{\omega}\left(x_{\omega}\right)\right\|}\right)^{d}-1\right)\right)^{1 / n} \\
& =1 / \varlimsup_{n \rightarrow \infty} \kappa(n)^{1 / n}=\kappa^{-1} .
\end{aligned}
$$

6.8. Remark. We have seen that if $\phi \equiv 0$ and $g \geq 0$, then $P$ behaves much like a positive operator, e.g. $r(P)$ is an eigenvalue of $P$. But since $\mathscr{H}^{\infty}(\mathscr{A})$ is far from being a Banach lattice, the general theory of positive operators does not apply. However, just as in the Banach lattice setting, one can show that if $\lambda$ is an eigenvalue of $P$ of modulus $r(P)$, then $\lambda^{-1}$ is a pole of the resolvent $(\mathrm{Id}-z P)^{-1}$ whose order does not exceed the order of $r(P)^{-1}$, which we denote by $\sigma$. This follows easily from

$$
\begin{aligned}
G(\lambda, t, s) & :=t\left(\frac{1-t}{t}\right)^{s}\left(\operatorname{Id}-t \lambda^{-1} P\right)^{-1} \\
& =t\left(\frac{1-t}{t}\right)^{s} \sum_{n=0}^{\infty}\left(\frac{t}{\lambda}\right)^{n} P^{n} \\
& =\left(\frac{1-t}{t}\right)^{s}\left(\sum_{\lambda_{i} \in \Gamma} \frac{\lambda}{\lambda_{i}} \sum_{k=0}^{\infty}\left(\frac{t \lambda_{i}}{\lambda-t \lambda_{i}}\right)^{k+1} E_{i} N_{i}^{k}\right. \\
& +(\text { terms bounded as } t \rightarrow 1))
\end{aligned}
$$

in the limit $t \rightarrow 1$ for $s>\sigma$ : Let $0 \leq f \in \mathscr{H}^{\infty}(\mathscr{A})$. Then

$$
\lim _{t \rightarrow 1}|G(\lambda, t, s)(f)| \leq \lim _{t \rightarrow 1} G(r(P), t, s)(f)=0,
$$

and for general $f \in \mathscr{H}^{\infty}(\mathscr{A})$ this estimate can be applied separately to the positive functions $f^{*}=\sum_{D \in \mathscr{D}}\left\|f_{D}\right\|_{\mathscr{A}} \cdot \chi_{D}$ and $\left(f+f^{*}\right)$.

Also, if $0 \leq f \in \mathscr{H}^{\infty}(\mathscr{A}), D \in \mathscr{D}$, and $\left(E_{1} N_{1}^{s+1} f\right)_{\mid D}=0$ for some $s \geq 0$, then $\left(E_{1} N_{1}^{s} f\right)_{\mid D} \geq 0$.

In Theorem 6.1(d) we have seen that, if $P$ is a Perron-Frobenius operator with respect to a measure $m$ with $\operatorname{supp}(m)=X$, then the positive cone of range $\left(E_{1}\right)$ is just the space of densities of $m$-absolutely continuous $T$-invariant measures. 
In general, the relation between $\operatorname{range}\left(E_{1}\right)$ and $T$-invariant measures is more complicated, even if $P=P_{g}$. For its investigation we need

6.9. Lemma. Suppose that $g \geq 0$, that $P=P_{g}$ is quasicompact, that $(X, T, \mathscr{Z})$ generates, and that $s$ is continuous. If $\sigma$ is the order of the pole $r(P)^{-1}$ of the resolvent of $P$, then there is a nonnegative, range $\left(E_{1}\right)$-valued Borel measure $F$ on $X$ such that

$$
\begin{aligned}
E_{1} N_{1}^{\sigma-1}(f)=\int f d F & \text { for all } f \in \mathscr{H}^{\infty}(\mathscr{A}), \\
\int P f d F=r(P) \cdot \int f d F & \text { for all } f \in L_{F}^{1}, \text { and } \\
\||F(Z)|\|_{\mathscr{A}} \leq\left\|E_{1} N_{1}^{\sigma-1}\right\|_{\mathscr{A}} & \text { for all } Z \in \mathscr{Z} .
\end{aligned}
$$

Proof. Without loss of generality assume $r(P)=1$. Since $P^{k} \chi_{Z} \in \mathscr{H}^{\infty}(\mathscr{A})$ for $Z \in \mathscr{Z}_{k}$,

$$
F(Z):=E_{1} N_{1}^{\sigma-1} P^{k}\left(\chi_{Z}\right)
$$

is well defined for such $Z, F \geq 0$ by Remark 6.8. It is easy to check that $F(Z)=\sum F\left(Z^{\prime}\right)$ where the sum extends over all $Z^{\prime} \in \mathscr{Z}_{k+1}$ with $Z^{\prime} \subseteq Z$. Hence $F$ extends uniquely to a finitely additive nonnegative set function on the algebra generated by $\bigcup_{k \geq 0} \mathscr{Z}_{k}$. Since all sets in this algebra are compact, $F$ is countably additive, and since $\|F(Z)\|_{\mathscr{A}} \leq\left\|E_{1} N_{1}^{\sigma-1}\right\| \|_{\mathscr{A}}$ for all $Z \in \mathscr{Z}, F$ extends to a nonnegative range $\left(E_{1}\right)$-valued measure on the $\sigma$-algebra generated by $\bigcup_{k \geq 0} \mathscr{Z}_{k}$, i.e. on the Borel sets.

Let $f \in \mathscr{H}^{\infty}(\mathscr{A}), D \in \mathscr{Z}$. For $k \geq 0$ and $Z \in \mathscr{Z}_{k}$ fix $\alpha_{Z} \in\left(f \cdot \chi_{D}\right)(Z)$ and consider $f_{k}:=\sum_{Z \in \mathscr{Z}_{k}} \alpha_{k} \chi_{Z} \cdot\left(f \cdot \chi_{D}\right)$ is continuous by continuity of $s$. Therefore $\left\|f \cdot \chi_{D}-f_{k}\right\|_{\infty}=: \delta_{k} \rightarrow 0$ as $k \rightarrow \infty$ and

$$
\begin{aligned}
\int_{D} f d F & =\lim _{k \rightarrow \infty} \int f_{k} d F=\lim _{k \rightarrow \infty} E_{1} N_{1}^{\sigma-1} P^{k} f_{k} \\
& \leq \lim _{k \rightarrow \infty} E_{1} N_{1}^{\sigma-1} P^{k}\left(\left(f+\delta_{k}\right) \chi_{D}\right) \\
& =E_{1} N_{1}^{\sigma-1}\left(f \cdot \chi_{D}\right),
\end{aligned}
$$

and for the reverse inequality consider $-f$ instead of $f$.

Since $\int P \chi_{Z} d F=E_{1} N_{1}^{\sigma-1} P^{k+1}\left(\chi_{Z}\right)=E_{1} N_{1}^{\sigma-1} P^{k}\left(\chi_{Z}\right)=\int \chi_{Z} d F$ for all $Z \in \mathscr{Z}_{k}, k \geq 0$, we also have $\int P f d F=\int f d F$ for all $f \in L_{F}^{1}$.

Next we have to think about the structure of the graph $(\mathscr{D}, \rightarrow):$ A subset $\mathscr{J}$ of $\mathscr{D}$ is irreducible, if for all $C, D \in \mathscr{I}$ there is a path from $C$ to $D$ and if $\mathscr{I}$ is maximal with respect to this property. It is well known that there is a maximal positive integer $p$ (the period of $\mathscr{I}$ ) such that the length of each closed path in $\mathscr{I}$ is a multiple of $p$ (see e.g. [Seneta, 1980]).

6.10. Proposition. Suppose that $P=P_{g}$ is quasicompact, that $(X, T, \mathscr{Z})$ generates, that $s$ is continuous, that $(\mathscr{D}, \rightarrow)$ is irreducible with period $p$ and that 
$g>0$ everywhere. Then

(a) The set $\Gamma$ of eigenvalues of $P$ of modulus $r(P)$ consists of the simple eigenvalues $r(P) \cdot e^{2 \pi i k / p} \quad(k=0, \ldots, p-1)$.

(b) There are a Borel measure $m$ on $X$ with $\operatorname{supp}(m)=X$ and $\sup _{D \in \mathscr{D}} m(D)<\infty$, and a strictly positive function $h \in \mathscr{H}^{\infty}(\mathscr{A})$ such that

$$
\begin{gathered}
E_{1} f=\int f d m \cdot h \quad \text { for all } f \in \mathscr{H}^{\infty}(\mathscr{A}), \text { and } \\
\int P f d m=r(P) \cdot \int f d m \text { for all } f \in L_{m}^{1} .
\end{gathered}
$$

The measure $\mu=h \cdot m$ is an ergodic $T$-invariant probability measure.

Proof. Let $\sigma$ be the order of the pole $r(P)$ of the resolvent of $P$ and denote $E=E_{1}, N=N_{1}$. Then $N^{\sigma}=0$ but $N^{\sigma-1} \neq 0$. Without loss of generality assume $r(P)=1$. Then $E N^{\sigma-1}=\lim _{t \rightarrow 1} G(1, t, \sigma) \geq 0$ by Remark 6.8, and $E N^{\sigma-1} P=E N^{\sigma-1}$.

Fix $C \in \mathscr{D}$ and let $j$ be the maximal integer such that $E N^{j} \chi_{C} \neq 0$. Obviously $j<\sigma$. Suppose for a contradiction that $j \geq 1$. Then $E N^{\sigma-1}\left(E N^{j} \chi_{C}\right)=$ 0 , where $E N^{j} \chi_{C} \geq 0$ by Remark 6.8. As $(X, T, \mathscr{Z})$ generates, there are $\varepsilon>0$ and $Z \in \mathscr{Z}_{n}$ with $\left(E N^{j} \chi_{C}\right)_{\mid Z} \geq \varepsilon$. Let $\omega$ be a path of length $k \geq n$ in $\mathscr{D}$ such that $Z(\omega) \subseteq Z$. Since $T^{k} Z(\omega)=: D \in \mathscr{D}$ and $\varepsilon_{k}:=\varepsilon \cdot \inf g_{k \mid Z(\omega)}>0$, we have

$$
\left(P^{k} E N^{j} \chi_{C}\right)_{\mid D} \geq\left(\left(E N^{j} \chi_{C}\right) \cdot g_{k}\right) \circ \tau_{\omega} \geq \varepsilon_{k} \cdot \chi_{D}
$$

Hence

$$
0=E N^{\sigma-1}\left(E N^{j} \chi_{C}\right)=E N^{\sigma-1} P^{k} E N^{j} \chi_{C} \geq \varepsilon_{k} \cdot E N^{\sigma-1} \chi_{D} \geq 0
$$

i.e. $E N^{\sigma-1} \chi_{D}=0$. This argument applies to all $D \in \mathscr{D}$, because $(\mathscr{D}, \rightarrow)$ is irreducible, in contradiction to $E N^{\sigma-1} \neq 0$. Hence $j=0$, i.e. $N \chi_{C}=0$, and as $C \in \mathscr{D}$ was arbitrary, $N=0$ and $E \geq 0$. In view of Remark 6.8 all eigenvalues in $\Gamma$ are semisimple.

We prove the simplicity of $r(P)=1$ : Let $P f=f$ for some $f \in C(X, \mathbb{R})$, and assume that $f(x)>0$ for some $x \in D \in \mathscr{D}$. As $(X, T, \mathscr{Z})$ generates, there are $n>0$ and $Z \in \mathscr{Z}_{n}$ such that $f_{\mid Z}>0$. Now the irreducibility of $(\mathscr{D}, \rightarrow)$ together with $g>0$ implies $f>0$ on all of $X$. Hence, if $P f=f$ for $f \in C(X, \mathbf{R})$, then $f=0, f<0$ or $f>0$ everywhere. If there are nonzero $f_{1}$ and $f_{2}$ as above, then $\psi_{\alpha}=f_{1}+\alpha f_{2}$ satisfies the assumptions on $f$ above, and since $\alpha^{-1} \psi_{\alpha} \rightarrow f_{2}$ as $\alpha \rightarrow \pm \infty$, there is some $\alpha \in \mathbf{R}$ with $\psi_{\alpha}=0$, i.e. $f_{1}=-\alpha f_{2}$. If $\stackrel{P}{P} h=h$ for some $h \in \mathscr{H}^{\infty}(\mathscr{A})$, then $\Re h$ and $\Im h$ satisfy the assumptions on $f$, because $s$ is continuous. Hence $\operatorname{rank}(E)=1$, i.e. $r(P)=1$ is a simple eigenvalue. The proof that $\Gamma=\left\{e^{2 \pi i k / p}: k=0, \ldots, p-1\right\}$ and that all $\lambda \in \Gamma$ are simple is postponed. 
Now fix $0<h \in \operatorname{range}(E)$. By Lemma 6.9 there is a Borel measure $m$ on $X$ such that $E f=h \cdot \int f d m$ and $\int P f d m=r(P) \cdot \int f d m$ for all $f \in \mathscr{C}^{\infty}(\mathscr{A})$ and $m(D) \leq\|E\|_{\mathscr{A}}$ for all $D \in \mathscr{Z}$.

We show $\operatorname{supp}(m)=X: m(D)>0$ for all $D \in \mathscr{D}$, because $(\mathscr{D}, \rightarrow)$ is irreducible and $m(D)=\int P^{n} \chi_{D} d m$ for all $n \geq 0$. Hence, if $Z=Z(\omega) \in$ $\mathscr{Z}_{n}$, then $m(Z)=\int P^{n} \chi_{Z} d m \geq \inf g_{n \mid Z} \cdot m\left(T^{n} Z\right)>0$ since $T^{n} Z \in \mathscr{D}$. As $(X, T, \mathscr{Z})$ generates, this proves the claim.

Finally, if $f \in L_{m}^{\infty}$, then

$$
\begin{aligned}
\int f \circ T d \mu & =\int f \circ T \cdot h d m=\int P(f \circ T \cdot h) d m \\
& =\int f \cdot P h d m=\int f \cdot h d m \\
& =\int f d \mu,
\end{aligned}
$$

and the ergodicity of $\mu$ follows immediately from $\operatorname{rank}(E)=1$. This proves (b).

We turn back to (a). Write $C \rightarrow{ }_{k} D$ if there is a path of length $k$ from $C$ to $D .\left(\mathscr{D}, \rightarrow_{k}\right)$ is again a directed graph. Assume first that $p=1$. Then $\left(\mathscr{D}, \rightarrow_{k}\right)$ is irreducible and of period 1 for each $k \geq 1$. Hence $r(P)=1$ is a simple eigenvalue for all $P^{k}$. With the measure $m$ constructed above we are in the situation of (c) and (d) of Theorem 6.1, in particular $\Gamma$ is a finite group. If there were $1 \neq \lambda \in \Gamma$, one would have $\lambda^{k}=1$ for some $k>1$ contradicting the simplicity of the eigenvalue 1 of $P^{k}$. Hence $\Gamma=\{1\}$ in case $p=1$.

If $p>1$, then $\mathscr{D}$ can be decomposed uniquely into $p$ disjoint subsets $\mathscr{D}_{0}, \ldots, \mathscr{D}_{p-1}$, each having the property that $C \in \mathscr{D}_{i}$ and $C \rightarrow_{k} D$ implies $D \in \mathscr{D}_{(i+k) \bmod p}$. The graphs $\left(\mathscr{D}_{i}, \rightarrow_{p}\right)$ are irreducible and of period $1(i=$ $0, \ldots, p-1)$. This is proved just as in the case of nonnegative matrices, see e.g. [Seneta, 1980]. Hence (a) and (b) of this proposition apply to each $P_{\mid V_{i}}^{p}$ where $V_{i}=\left\{f \in \mathscr{C}^{\infty}(\mathscr{A}): f_{D} \equiv 0\right.$ if $\left.D \notin \mathscr{D}_{i}\right\}$. In particular $P_{\mid V_{i}}^{p}$ has only 1 as an eigenvalue of modulus one, and its multiplicity is $p$. This implies immediately that $\sum_{\lambda \in \Gamma}$ multiplicity $(\lambda)=p$, and since $\Gamma$ is a group, we can finish the proof by showing that $e^{-2 \pi i / p} \in \Gamma$ : Let $\psi(x)=k$ if $x \in D \in \mathscr{D}_{k}$ and let $P h=h$. Then $P\left(h e^{2 \pi i \psi / p}\right)=e^{2 \pi i(\psi-1) / p} \cdot P(h)=e^{-2 \pi i / p} \cdot h \cdot e^{2 \pi i \psi / p}$, i.e. $e^{-2 \pi i / p} \in \Gamma$.

Now we turn to the case of a general graph $(\mathscr{D}, \rightarrow)$. Let $\mathscr{J}_{1}, \mathscr{I}_{2}, \ldots$ be the irreducible subsets of $\mathscr{D}, X_{i}:=\bigcup_{D \in \mathcal{F}_{i}} D$ for each $i$. Let $Y_{i}:=\bigcap_{n \geq 0} T^{-n} X_{i}$. Since the $\mathscr{F}_{i}$ are irreducible, $T Y_{i}=Y_{i}$, and it is easily checked that

$$
\mathscr{Z}_{i}=\left\{D \cap Y_{i}: D \in \mathscr{F}_{i}, D \cap Y_{i} \neq \varnothing\right\}
$$


is a Markov partition for $T_{\mid Y_{i}} \cdot\left(Y_{i}, T, \mathscr{Z}_{i}\right)$ generates if $(X, T, \mathscr{Z})$ does. We make the following additional assumption:

If $\left(\mathscr{J}_{i}, \rightarrow\right)$ does not consist of a single loop only, and if $D \cap Y_{i} \neq \varnothing$, then $s\left(D \cap Y_{i}\right)$ is a uniqueness set for bounded holomorphic functions on $U_{D}$.

This strengthens requirement (ii) of Definition 5.2. One should note, however, that $D \cap Y_{i}$ is uncountable if $\left(\mathscr{I}_{i}, \rightarrow\right)$ does not consist of a single loop only, and hence $(6.11)$ is not really restrictive.

Under this assumption $\left(Y_{i}, T, \mathscr{Z}_{i}, g\right)$ is $\mathscr{A}_{i}$-expanding if $(X, T, \mathscr{Z}, g)$ is $\mathscr{A}$-expanding where $\mathscr{A}_{i}=\left(s,\left(U_{D}\right)_{D \in \mathcal{F}_{1}}\right)$. The transfer operator corresponding to $\left(Y_{i}, T, \mathscr{Z}_{i}, g\right)$ is denoted by $P_{i}$.

6.11. Theorem. Suppose that $(X, T, \mathscr{Z}, g)$ satisfies $(6.11)$ and the assumptions of Theorem 6.2, that $r_{\text {as }}<r(P)$ and $\gamma_{\text {as }}<\gamma(\mathscr{D})$, that $g>0$ everywhere, and that $s$ is continuous. Let $P=P_{g}$ and $d=\operatorname{rank}\left(E_{1}\right)$. Then $r\left(P_{i}\right)<r(P)$ except for $d$ irreducible subsets of $\mathscr{D}\left(\right.$ say $\left.\mathscr{I}_{1}, \ldots, \mathscr{I}_{d}\right)$ for which $r\left(P_{i}\right)=r(P)$ and for which holds:

(a) The subsystem $\left(Y_{i}, T, \mathscr{Z}_{i}, g\right)$ satisfies all assumptions of Theorems 6.1 and 6.2 and Proposition 6.10, and it does not consist of a single loop only.

(b) There are a $\sigma$-finite Borel measure $m_{i}$ on $X$ with $\operatorname{supp}\left(m_{i}\right)=Y_{i}$ and a function $0 \leq h_{i} \in \mathscr{H}^{\infty}(\mathscr{A})$, zero outside $X_{i}$, satisfying

$$
\left(E_{1}\left(f \cdot \chi_{X_{i}}\right)\right)_{\mid X_{i}}=\int_{X_{i}} f d m_{i} \cdot h_{i \mid X_{t}} \text { for all } f \in \mathscr{H}^{\infty}(\mathscr{A}) .
$$

The measure $\mu_{i}=h_{i} \cdot m_{i}$ is a $T$-invariant ergodic probability measure equivalent to $m_{i} \cdot h_{i}$ satisfies $\left(P h_{i}\right)_{\mid X_{1}}=h_{i \mid X_{i}}$.

(c) There is a $\sigma$-finite Borel measure $\bar{m}_{i}$ on $X$ such that $\bar{m}_{i \mid X_{i}}=m_{i \mid X_{i}}$,

$$
\int P f d \bar{m}_{i}=r(P) \cdot \int f d \bar{m}_{i} \quad \text { for all } f \in L_{\bar{m}_{i}}^{1}
$$

and $\operatorname{supp}\left(\bar{m}_{i}\right)=\operatorname{cl}\left(\bigcup_{k \geq 0} T^{-k} Y_{i}\right)$.

Proof. Fix some $\mathscr{J}_{i}$. If $\gamma\left(\mathscr{F}_{i}\right)<\gamma(\mathscr{D})=r(P)$, then $1 / \zeta\left(Y_{i}, z\right)$ is analytic and nonzero in $\left\{|z|<(r(P)-\varepsilon)^{-1}\right\}$ for some $\varepsilon>0$. In particular, $1 / r(P)$ is not a zero of $1 / \zeta\left(Y_{i}, z\right)$. Also $r\left(P_{i}\right)<r(P)$, since otherwise $r_{\text {as }}\left(P_{i}\right) \leq r_{\text {as }}(P)<$ $r(P)=r\left(P_{i}\right)$ and $\gamma_{\text {as }}\left(\mathscr{J}_{i}\right) \leq \gamma_{\text {as }}(\mathscr{D}) \leq \gamma(\mathscr{D})=r(P)=r\left(P_{i}\right)$ such that Theorem 6.2 would lead to the contradiction $r\left(P_{i}\right)=\gamma\left(\mathscr{I}_{i}\right)<\gamma(\mathscr{D})=r(P)$.

If, on the other hand, $r(P)=\gamma(\mathscr{D})=\gamma\left(\mathscr{F}_{i}\right)$, then $\gamma_{\text {as }}\left(\mathscr{I}_{i}\right) \leq \gamma_{\text {as }}(\mathscr{D})<$ $\gamma(\mathscr{D})=\gamma\left(\mathscr{F}_{i}\right)$ and $r_{\text {as }}\left(P_{i}\right) \leq r_{\text {as }}(P) \leq r(P)=\gamma\left(\mathscr{J}_{i}\right)$, and Theorem 6.2 applies to $P_{i}$. Hence $r\left(P_{i}\right)=\gamma\left(\mathscr{J}_{i}\right)=r(P)$, and in view of Proposition $6.10 r(P)$ is a simple eigenvalue of $P_{i}$, such that, by Theorem 6.2 again, $1 / r(P)$ is a simple zero of $1 / \zeta\left(Y_{i}, z\right)$.

As $\zeta(X, z)=\prod_{i} \zeta\left(Y_{i}, z\right)$, there are exactly $d$ irreducible components $\mathscr{J}_{i}$ of $(\mathscr{Z}, \rightarrow)$, say $\mathscr{J}_{1}, \ldots, \mathcal{F}_{d}$, for which $r\left(P_{i}\right)=r(P)$ is a simple eigenvalue of $P_{i}$. 
Now Proposition 6.10 guarantees the existence of measures $m_{i}$ and $\mu_{i}=$ $h_{i} \cdot m_{i}$ with full topological support on $Y_{i}$. Since the $Y_{i}$ are closed subsets of the $X_{i}$, these measures can be interpreted as measures on $X_{i}$ with topological support $Y_{i}$. Since $T Y_{i}=Y_{i}$, the $\mu_{i}$ are ergodic, $T$-invariant probability measures.

We use the operators $G(\lambda, t, s)$ defined in (6.10) and denote by $G_{i}(\lambda, t, s)$ the corresponding operators for the $P_{i}$. Observing (6.11) we see that $D$ and $D \cap Y_{i}$ have actually the same analytic structure for all $D \in \mathscr{J}_{i}$. Hence $\left(P^{n}\left(f \cdot \chi_{X_{i}}\right)\right)_{\mid Y_{i}}=P_{i}^{n}\left(f_{\mid Y_{i}}\right)$, in particular $\left(P h_{i}\right)_{Y_{i}}=P_{i}\left(h_{i \mid Y_{i}}\right)=h_{i \mid Y_{i}}$ and $\left(P h_{i}\right)_{\mid X_{i}}=h_{i \mid X_{i}}$. Thus for all $0<t<1$ and $s \geq 1$

$$
\left(G(r(P), t, s)\left(f \cdot \chi_{X_{i}}\right)\right)_{\mid Y_{i}}=G_{i}(r(P), t, s)\left(f_{\mid Y_{i}}\right) .
$$

But $\lim _{t \rightarrow 1} G_{i}(r(P), t, s)=0$ for $s \geq 2$, whence

$$
\begin{aligned}
& \left(E_{1}\left(f \cdot \chi_{X_{i}}\right)\right)_{\mid Y_{i}}=\lim _{t \rightarrow 1}\left(G(r(P), t, 1)\left(f \cdot \chi_{X_{i}}\right)\right)_{\mid Y_{i}} \\
& \quad=\lim _{t \rightarrow 1} G_{i}(r(P), t, 1)\left(f_{\mid Y_{i}}\right)=E_{i, 1}\left(f_{\mid Y_{i}}\right) \\
& \quad=\int_{Y_{i}} f d m_{i} \cdot h_{i}=\int_{X_{i}} f d m_{i} \cdot h_{i} \text { by Proposition 6.10. }
\end{aligned}
$$

In view of (6.11), $\int_{X_{i}} f d m_{i} \cdot h_{i}$ extends uniquely to the nonnegative function $E_{1}\left(f \cdot \chi_{X_{i}}\right)$ defined on $X_{i}$. This proves (a) and (b).

We turn to (c): For $k \geq 0$ let

$$
X_{i, k}=\left\{x \in X: T^{k} x \in X_{i}, T^{j} x \notin X_{i}(j=0, \ldots, k-1)\right\},
$$

and for a bounded measurable function $f$ let

$$
m_{i, k}(f):=m_{i}\left(P^{k}\left(f \cdot \chi_{i, k}\right)\right), \quad \text { where } \chi_{i, k}:=\chi_{X_{i, k}} .
$$

Then $\bar{m}_{i}:=\sum_{k \geq 0} r(P)^{-k} m_{i, k}$ is a $\sigma$-finite Borel measure, and using the relations

$$
\begin{aligned}
& P f \cdot \chi_{i, 0}=P\left(f \chi_{i, 0}+f \chi_{i, 1}\right), \\
& P f \cdot \chi_{i, k}=P\left(f \chi_{i, k+1}\right) \quad(k \geq 1)
\end{aligned}
$$

it is straightforward to check that $\bar{m}_{i}(P f)=r(P) \cdot \bar{m}_{i}(f)$ for $f \in L_{\bar{m}_{i}}^{1}$.

B. Examples.

6.12. Example I (topological Markov shifts, sofic systems).

For a finite state Markov shift whose transition matrix has a positive spectral radius Theorems 6.1 and 6.2 apply imediately, because $\gamma_{\text {as }}=r_{\text {as }}=0<r(P)$ and Id $\in \mathscr{F}$. (The latter assures condition (iii) of Theorem 6.2.)

If $\rho_{1}, \ldots, \rho_{d}$ are as in Example 5.9, then

$$
G_{\mathscr{F}}=\left\{z \in \mathbb{C}^{d}:|z| \cdot r(P)<\left(\prod_{j=1}^{d} \rho_{j}\right)^{-1}\right\} .
$$


Hence, the smaller the $\rho_{j}$ (i.e. the "smoother" the functions in $\mathscr{H}^{\infty}(\mathscr{A})$ ), the bigger is $G_{\mathscr{F}}$.

If $U_{D}=\mathbb{C}(D \in \mathscr{D})$, i.e. if $\mathscr{H}^{\infty}\left(U_{D}\right) \cong \mathbb{C}^{d}$ and $\mathscr{H}^{\infty}(\mathscr{A}) \cong \mathbb{C}^{d \cdot \operatorname{card}(\mathscr{D})}$, then Theorems 6.1 and 6.2 reduce to statements about matrices (cf. 4.8).

These results are well known, of course. The analyticity part of Theorem 6.2 (and much more) for finite state Markov shifts is due to [Ruelle, 1976].

For general countable state Markov shifts the assumptions of the theorems must be checked in each case individually.

As the canonical Markov extensions of sofic systems are finite state topological Markov chains, the above remarks apply to these extensions as well.

6.13. Example II (up-and-down-counter, cf. 3.13).

We consider the canonical Markov extension of the counter $\left(\Omega\left(W_{M}\right), S, \mathscr{Z}\right)$. Its Markov diagram is uniformly forward finite, since each $D \in \mathscr{D}$ has at most two successors. Fix an analytic structure $\mathscr{A}$ and a weight function $g$ such that $\left(\Omega\left(W_{M}\right), S, \mathscr{Z}, g\right)$ is an $\mathscr{A}$-expanding weighted system (cf. 5.2, 5.3, 5.9). Let $\mathscr{E}=\left\{E, F_{0}\right\}$ (cf. 3.13). As there are no closed paths in $\mathscr{D} \backslash \mathscr{E}$, we have $\gamma_{\text {as }}=\gamma(\mathscr{D} \backslash \mathscr{E})=0$. As the number of paths of length $n$ in $\mathscr{D} \backslash \mathscr{E}$ starting at any $D \in \mathscr{D} \backslash \mathscr{E}$ is bounded by $n$,

$$
r_{\text {as }} \leq \varlimsup_{n \rightarrow \infty}\left(n \cdot\left\|g_{n}\right\|_{\infty}\right)^{1 / n}=g_{\infty}
$$

for $\hat{P}$ acting on $\mathscr{H}^{\infty}(\mathscr{A})$. Hence Theorems 6.1 and 6.2 apply if $g_{\infty}<r(\hat{P})$ or $g_{\infty} \leq \gamma(\mathscr{D})$.

If $g \equiv 1$, then $r\left(\hat{P}_{\hat{g}}\right)>1=g_{\infty}$ as the diagram $(\mathscr{D}, \rightarrow)$ contains finite subdiagrams describing topological Markov shifts of positive entropy. In particular Theorem 6.2 applies in this case and $G_{\mathscr{F}} \supseteq\{|z|<1\}$ provided the $\rho_{j}$ from 5.9 have been chosen such that $\prod_{j=1}^{d} \rho_{j} \cdot \gamma(\mathscr{D}) \leq 1$ or $U_{D}=\mathbb{C}(D \in \mathscr{D})$. From the explicit formula for the zeta function (see Example 4.9) or from Proposition 6.10 it follows immediately that $\gamma(\mathscr{D})^{-1}=r(\hat{P})^{-1}$ is a simple pole of $\hat{\zeta}(z)$. Hence $\lambda_{1}=r(\hat{P})$ is a simple eigenvalue of $\hat{P}$.

If $M=\mathbb{N}$, then $\hat{\zeta}_{1}(z)=(1+z) /\left(1-2 z^{2}\right)$ (see 4.10), and $\{|z|<1\}$ is indeed the greatest ball around 0 to which $\hat{\zeta}_{1}^{-1}(z)$ can be analytically extended.

6.14. Example III (piecewise monotonic transformations with finitely many monotonicity intervals).

In order to check the assumptions made in Theorems 6.1 and 6.2 we need some informations from [Hofbauer, 1985, Theorem 9 and its Corollary 1] about the structure of the Markov diagram for piecewise monotonic transformations:

Let $\mathscr{D}_{0}=\mathscr{Z}$ and $\mathscr{D}_{n+1}=\mathscr{D}_{n} \cup\left\{D \in \mathscr{D}: \exists C \in \mathscr{D}_{n}\right.$ such that $\left.C \rightarrow D\right\}$ $(n \geq 1)$. Then

$$
\mathscr{D}_{n} \subseteq \mathscr{D}_{n+1}(n \geq 1), \quad \bigcup_{n \geq 1} \mathscr{D}_{n}=\mathscr{D}, \quad \operatorname{card}\left(\mathscr{D}_{n}\right)=O(n)
$$

$$
\lim _{n \rightarrow \infty} r\left(M_{\mid \mathscr{Z} \backslash \mathscr{Z}_{n}}\right)=1
$$


where $M_{\mid \mathscr{D} \backslash \mathscr{\mathscr { O } _ { n }}}$ is the transition matrix of $(\mathscr{D}, \rightarrow)$ restricted to entries from $\mathscr{D} \backslash \mathscr{D}_{n}$ and acting by right-multiplication on row vectors of $l^{1}\left(\mathscr{D} \backslash \mathscr{D}_{n}\right)$. Now let $\omega=D_{0} \cdots D_{n}$ be a path of length $n$ in $\mathscr{D}$. Then

$$
D_{n} \in \mathscr{D}_{k+n} \text { if } D_{0} \in \mathscr{D}_{k} \text {, and }
$$

$\omega$ is closed, then it is completely contained in $\mathscr{D}_{2 n}$ provided $n \geq n_{0}$ for some $n_{0} \in \mathbb{N}$.

As $\mathscr{Z}$ is finite, each $D \in \mathscr{D}$ has at most $\operatorname{card}(\mathscr{Z})$ successors, i.e. $(\mathscr{D}, \rightarrow)$ is uniformly forward finite.

Let $\mathscr{F}=\left\{\beta\left[\mathscr{D}_{n}\right]: n \in \mathbb{N}\right\}$. For $\beta=\beta\left[\mathscr{D}_{l}\right]$ and $\hat{f} \in \mathscr{H}^{\infty}(\mathscr{A})$ we have in view of $(6.3)$

$$
\left\|\hat{P}_{\beta}^{n} \hat{f}\right\|_{\mathscr{\infty}} \leq \text { const } \cdot\|\hat{f}\|_{\mathscr{A}} \cdot\left\|g_{n}\right\|_{\infty} \cdot\left\|\left(M_{\mid \mathscr{D} \backslash \mathscr{D}_{1}}\right)^{n}\right\|_{1},
$$

i.e. $r_{\beta} \leq g_{\infty} \cdot r\left(M_{\mid \mathscr{D} \backslash \mathscr{D}_{1}}\right)$, and therefore $r_{\text {as }}=\underline{\lim }_{\beta \in \mathscr{T}} r_{\beta} \leq g_{\infty}$ by (6.13). Hence $r_{\text {as }}<r(\hat{P})$ provided that $g_{\infty}<r(\hat{P})$. Similarly it follows from (6.15) that $\gamma_{\text {as }} \leq g_{\infty}$, cf. the proof of Lemma 5 in [Hofbauer/Keller, 1984].

We turn to the particular case where $\tilde{T}$ is piecewise analytic as discussed in Example 5.10 and where $\tilde{g}=1 /\left|\tilde{T}^{\prime}\right|$. Let $g$ be the "lift" of $\tilde{g}$ to the associated piecewise compact space $X$ (see $3.14,3.15$ ). Choosing $\varepsilon>0$ in 5.10 small enough, $(X, T, \mathscr{Z}, g)$ becomes a weighted $\mathscr{A}$-expanding system. In particular $r(\hat{P}) \geq 1$ as $\int \hat{P} \hat{f} d \hat{m}=\int \hat{f} d \hat{m}$ (where $\hat{m}$ is the Lebesgue measure lifted to the Markov extension $\hat{X}$ of $X$ ). Hence Theorem 6.1 applies if $g_{\infty}<1$.

Assumptions (i) and (ii) of Theorem 6.2 are now trivially sastisfied as

$$
\operatorname{card}\left(\left\{\hat{x}: \hat{T}^{n} \hat{x}=\hat{x}\right\}\right) \leq(\operatorname{card}(\mathscr{Z}))^{n} .
$$

We check assumption (iii): Let $\beta=\beta\left[\mathscr{D}_{k}\right], n \in \mathbf{N}$, and $\alpha=\beta\left[\mathscr{D}_{k+n}\right]$. Then, in view of (6.14), $\alpha\left(P_{\beta} \alpha\right)^{j} P \beta=P_{\beta}^{j} P \beta$ for $0 \leq j<n$.

We summarize the results for the case $\tilde{g}=1 /\left|\tilde{T}^{\prime}\right|: \quad r_{\text {ess }}=r_{\text {as }} \leq g_{\infty}, \gamma_{\text {as }} \leq$ $g_{\infty}, \gamma(\mathscr{D})=r(\hat{P})=1$, and hence $\kappa \leq g_{\infty} \gamma(\mathscr{D})=g_{\infty}$. In particular $G_{\mathscr{P}} \supseteq$ $\left\{|z|<g_{\infty}^{-1}\right\}$. We also note that in this case

$$
1=\gamma(\mathscr{D})=\varlimsup_{n \rightarrow \infty}\left(\sum_{\omega}\left\|D \tau_{\omega}\left(x_{\omega}\right)\right\|\right)^{1 / n}
$$

where the sum extends over all closed paths $\omega$ of length $n$. If $\tilde{g} \neq 1 /\left|\tilde{T}^{\prime}\right|$, then (6.16) still allows the conclusion $\kappa \leq g_{\infty} \cdot \gamma(\mathscr{D})=g_{\infty}$, and hence again $G_{\mathscr{F}} \supseteq\left\{|z|<g_{\infty}^{-1}\right\}$. 


\section{EIGENVALUES AND EIGENFUNCTIONS FOR NON-MARKOVIAN SYSTEMS}

\section{A. A general result.}

Suppose that $(X, T, \mathscr{Z}, g)$ is a weighted, $\mathscr{A}$-expanding, piecewise compact system (not necessarily Markovian) and that $(\hat{X}, \hat{T}, \hat{Z}, \hat{g})$ is its canonical Markov extension. Our goal is to derive knowledge about eigenvalues and eigenfunctions of $P_{g}$ and poles of $\zeta_{g}(z)$ from corresponding results for $\hat{P}_{\hat{g}}$ and $\hat{\zeta}_{\hat{g}}(z)$ proved in the last section.

$\zeta_{g}(z)$ and $\hat{\zeta}_{\hat{g}}(z)$ have been related in Lemma 4.7 and Examples 4.8-4.10, and it seems difficult to prove general results about this relation more precise than Lemma 4.7.

$P_{g}$ and $\hat{P}_{\hat{g}}$ are related by

$$
\pi_{*} \circ \hat{P}_{\hat{g}}=P_{g} \circ \pi_{*} \quad(\text { Lemma 4.6) }
$$

This identity, however, is of no use, unless one has some additional information about the range of $\pi_{*}: \mathscr{H}^{\infty}(\mathscr{A}) \rightarrow \mathrm{mb}(X)$.

In order to state a rather general result, which applies to various examples, we introduce the following notation:

Let $P=P_{g}, \hat{P}=\hat{P}_{\hat{g}}$, and suppose

(A1) $(\hat{H},|\|||\|$.$) is a Banach space of (equivalence classes of functions on$ $\hat{X}, \hat{P}(\hat{H}) \subseteq \hat{H}$.

(A2) $(H,\|\|$.$) is a Banach space of (equivalence classes of) functions on X$, $P(H) \subseteq H$.

(A3) $\pi_{*}(\hat{H}) \subseteq H$ and $\pi_{*}: \hat{H} \rightarrow H$ is continuous.

(A4) $\hat{P}: \hat{H} \rightarrow \hat{H}$ is quasicompact with spectral radius $r(\hat{P})$ and essential spectral radius $\hat{r}_{\text {ess }}$, and for $\hat{r}>\hat{r}_{\text {ess }}$ there is $N=N(\hat{r}) \in \mathbb{N}$ such that

$$
\hat{P}=\sum_{i=1}^{N} \lambda_{i}\left(\hat{E}_{i}+\hat{N}_{i}\right)+\hat{Q}
$$

as in (2.2). We write $\hat{E}_{\lambda}=\hat{E}_{i}$ if $\lambda=\lambda_{i}$ and $\hat{E}_{\lambda}=0$ otherwise.

(A5) $P: H \rightarrow H$ is bounded with spectral radius $r(P)$.

Under these assumptions we can state

\subsection{Proposition.}

(a) Assume that $\left\|\hat{P}^{n}\right\|=O\left(r(\hat{P})^{n}\right),\left\|P^{n}\right\|=O\left(r(P)^{n}\right)$, and that $\pi_{*}(\hat{H})$ is dense in $H$. If $(P-\lambda \mathrm{Id})^{k} f=0$ for some $f \in H, k \geq 1,|\lambda|=r(P)$, then $P f=\lambda f$, and there is $\hat{f} \in \hat{H}$ with $\pi_{*} \hat{f}=f$ and $\hat{P} \hat{f}=\lambda \hat{f}$. Briefly

$$
\bigcup_{k \geq 1} \operatorname{kernel}\left((P-\lambda \mathrm{Id})^{k}\right)=\operatorname{kernel}(P-\lambda \mathrm{Id})=\pi_{*}\left(\hat{E}_{\lambda}(\hat{H})\right) \text {. }
$$


(b) If $(P-\lambda \text { Id })^{k} f=0$ for some $f \in H, k \geq 1,|\lambda|>\hat{r}_{\text {ess }}$, and if condition (A6) below is satisfied, then there is $\hat{f} \in \hat{H}$ with $\pi_{*} \hat{f}=f$ and $\hat{E}_{\lambda} \hat{f}=\hat{f}$. Briefly

$$
\bigcup_{k \geq 1} \operatorname{kernel}\left((P-\lambda \mathbf{I d})^{k}\right)=\pi_{*}\left(\hat{E}_{\lambda}(\hat{H})\right) \text {. }
$$

The condition mentioned above is:

(A6) There are a sequence of functions $\hat{f}_{n} \in \operatorname{mb}(\hat{X})$ and constants $\alpha, \beta$, $\gamma_{1}, \gamma_{2}, C \geq 0$ such that for $m=[\beta n]$

$$
\begin{gathered}
\hat{P}^{m} \hat{f}_{n} \in \hat{H}, \quad\left\|\hat{P}^{m} \hat{f}_{n}\right\| \leq C \cdot \gamma_{1}^{n}, \quad\left\|f-\pi_{*} \hat{f}_{n}\right\| \leq C \cdot \gamma_{2}^{n}, \\
\gamma_{1}<\frac{|\lambda|^{\alpha+\beta}}{\hat{r}_{\text {ess }}^{\alpha}}, \quad \gamma_{2}<\left|\frac{\lambda}{r(P)}\right|^{\alpha+\beta} .
\end{gathered}
$$

7.2. Remark. If $f \in \pi_{*}(\hat{H})$, one may choose $\hat{f}_{n}=\hat{f}, \alpha=1, \beta=0, \gamma_{1}=1$, $\gamma_{2}=0$ in (A6), and the condition reduces to $|\lambda|>\hat{r}_{\text {ess }}$. This is always the case if $e_{*}(H) \subseteq \hat{H}$.

Proof of the proposition.

(a) Define $S_{\lambda, n}=\frac{1}{n} \sum_{j=0}^{n-1}\left(\lambda^{-1} P\right)^{j}$ and $\hat{S}_{\lambda, n}$ analogously. As $\left\|\hat{P}^{n}\right\|=$ $O\left(r(\hat{P})^{n}\right), \lim _{n \rightarrow \infty} \hat{S}_{\lambda, n}=\hat{E}_{\lambda}$ strongly and $\hat{P} \hat{E}_{\lambda}=\lambda \hat{E}_{\lambda}$ (see Corollary VIII.5.2 in [Dunford/Schwartz, 1958]). Hence

$$
\lim _{n \rightarrow \infty} S_{\lambda, n} \circ \pi_{*}=\lim _{n \rightarrow \infty} \pi_{*} \circ \hat{S}_{\lambda, n}=\pi_{*} \circ \hat{E}_{\lambda}
$$

strongly. Since $\pi_{*}(\hat{H})$ is dense in $H$ and $\left\|P^{n}\right\|=O\left(r(P)^{n}\right)$, we may assume $P f=\lambda f$, and there are $\hat{f}_{j} \in \hat{H}$ with $\left\|f-S_{\lambda, n}\left(\pi_{*} \hat{f}_{j}\right)\right\| \leq j^{-1}$ for all $n$. Hence $\left\|f-\pi_{*}\left(\hat{E}_{\lambda} \hat{f}_{j}\right)\right\| \leq j^{-1}$, and as range $\left(\pi_{*} \circ \hat{E}_{\lambda}\right)$ is finite-dimensional, $f \in \operatorname{range}\left(\pi_{*} \circ \hat{E}_{\lambda}\right)$. This proves (a).

(b) In view of (A6) we can fix $\hat{r}>\hat{r}_{\text {ess }}$ and $r>r(P)$ such that

$$
\hat{r}<|\lambda|, \quad \gamma_{1}<\frac{|\lambda|^{\alpha+\beta}}{\hat{r}^{\alpha}}, \quad \gamma_{2}<\left|\frac{\lambda}{r}\right|^{\alpha+\beta} .
$$

We may assume that $\hat{Q}$ from (A4) has spectral radius less than $\hat{r}$.

Suppose now

$$
(P-\lambda \mathbf{I d}) f=h=\pi_{*} \hat{h} \in \pi_{*}\left(\hat{E}_{\lambda}(\hat{H})\right) .
$$

We shall show

$$
f \in \pi_{*}\left(\hat{E}_{\lambda}(\hat{H})\right) .
$$

Observe first that (7.2) implies

$$
\left(\lambda^{-1} P\right)^{n} f-f=\lambda^{-1} \sum_{j=0}^{n-1}\left(\lambda^{-1} P\right)^{j} h \in \pi_{*}\left(\hat{E}_{\lambda}(\hat{H})\right)
$$

because $\left(P^{j} \circ \pi_{*} \circ \hat{E}_{\lambda}\right)(\hat{H})=\left(\pi_{*} \circ \hat{P}^{j} \hat{E}_{\lambda}\right)(\hat{H})=\left(\pi_{*} \circ \hat{E}_{\lambda} \hat{P}^{j}\right)(\hat{H}) \subseteq \pi_{*}\left(\hat{E}_{\lambda}(\hat{H})\right)$. 
Let $l=[\alpha n]+1$ and define

$$
\hat{u}_{n}=\left(\lambda^{-1} \hat{P}-\lambda^{-1} \hat{Q}\right)^{l}\left(\lambda^{-1} \hat{P}\right)^{m} \hat{f}_{n} .
$$

$\hat{u}_{n}$ is an element of the finite-dimensional space $\hat{W}:=\operatorname{range}\left(\sum_{i=1}^{N} \hat{E}_{i}\right), \hat{E}_{i}$ as in (A4).

Note that $\hat{E}_{\lambda}(\hat{H}) \subseteq \hat{W}$. Now

$$
\begin{aligned}
\| f- & \left(f-\left(\lambda^{-1} P\right)^{l+m} f\right)-\pi_{*}\left(\hat{u}_{n}\right) \| \\
& \leq\left\|\left(\lambda^{-1} P\right)^{l+m}\left(f-\pi_{*} \hat{f}_{n}\right)\right\|+\left\|\pi_{*}\left(\left(\lambda^{-1} \hat{P}\right)^{l+m} \hat{f}_{n}-\hat{u}_{n}\right)\right\| \\
& \leq \text { const } \cdot\left|\frac{r}{\lambda}\right|^{l+m} \cdot \gamma_{2}^{n}+\text { const } \cdot\left\|\left(\lambda^{-1} \hat{Q}\right)^{l}\left(\lambda^{-1} \hat{P}\right)^{m} \hat{f}_{n}\right\| \\
& \leq \text { const } \cdot\left(\left|\frac{r}{\lambda}\right|^{\alpha+\beta} \cdot \gamma_{2}\right)^{n}+\text { const } \cdot \hat{r}^{l}|\lambda|^{-(l+m)} \gamma_{1}^{n} \\
& \leq \text { const } \cdot\left(\left(\left|\frac{r}{\lambda}\right|^{\alpha+\beta} \cdot \gamma_{2}\right)^{n}+\left(\frac{\hat{r}^{\alpha}}{|\lambda|^{\alpha+\beta}} \gamma_{1}\right)^{n}\right) \\
& \longrightarrow 0 \quad \text { as } n \rightarrow \infty \quad \text { by }(7.1) .
\end{aligned}
$$

Hence $f$ can be approximated by elements from $\pi_{*}(\hat{W})$ (see (7.4)), and as $\operatorname{dim}\left(\pi_{*}(\hat{W})\right) \leq \operatorname{dim}(\hat{W})<\infty$, we have $f \in \pi_{*}(\hat{W})$, say $f=\pi_{*} \hat{w}, \hat{w} \in \hat{W}$.

Let $\hat{K}=\left\{\hat{w} \in \hat{W}: \pi_{*}(\hat{w})=0\right\}$. As $\pi_{*} \circ \hat{P}=P \circ \pi_{*}$, we have

$$
(\hat{P}-\lambda \mathrm{Id}) \hat{K} \subseteq \hat{K}
$$

Let $\hat{U}=\hat{E}_{\lambda}(\hat{H})$ and $\hat{V}=\left(\sum_{\substack{i=1, \ldots, N \\ \lambda_{1} \neq \lambda}} \hat{E}_{\lambda_{1}}\right)(\hat{H})$. Then $\hat{U}+\hat{V}=\hat{W}$, $\hat{U} \cap \hat{V}=\{0\}$, and both spaces are stable under $(\hat{P}-\lambda$ Id $) . \quad(\hat{P}-\lambda \text { Id })_{\mid \hat{V}}$ is an isomorphism of the finite-dimensional space $\hat{V}$ and hence also of its stable subspace $\hat{V} \cap \hat{K}$.

Let $\hat{f}=\hat{u}+\hat{v}, \hat{u} \in \hat{U}, \quad \hat{v} \in \hat{V}$. For large $s$ we have

$$
\begin{aligned}
\pi_{*}(\hat{P}-\lambda \mathrm{Id})^{s} \hat{v} & =\pi_{*}(\hat{P}-\lambda \mathrm{Id})^{s} \hat{w}=(P-\lambda \mathrm{Id})^{s} f \\
& =(P-\lambda \mathrm{Id})^{s-1} \pi_{*} \hat{h}=\pi_{*}(\hat{P}-\lambda \mathrm{Id})^{s-1} \hat{h}=0 .
\end{aligned}
$$

This shows that $(\hat{P}-\lambda \text { Id })^{s} \hat{v}$ and hence also $\hat{v}$ belong to $\hat{V} \cap \hat{K}$. Therefore $f=\pi_{*} \hat{w}=\pi_{*} \hat{u} \in \pi_{*}\left(\hat{E}_{\hat{\lambda}}(\hat{H})\right)$, and (7.3) is proved.

With $h \equiv 0$ in (7.2) this proves (b) of the proposition for the case $k=1$.

For $k \geq 1$ we proceed by induction:

Suppose we know that for some $k \geq 1$

$$
(P-\lambda \mathrm{Id})^{k} f \in \pi_{*}\left(\hat{E}_{\dot{\lambda}}(\hat{H})\right)
$$

implies

$$
f \in \pi_{*}\left(\hat{E}_{j}(\hat{H})\right) .
$$

For $k=1$ this has just been proved $((7.2) \Rightarrow(7.3))$. 
If $(P-\lambda \text { Id })^{k+1} f \in \pi_{*}\left(\hat{E}_{\lambda}(\hat{H})\right)$, then $f_{1}:=(P-\lambda$ Id $) f$ satisfies (7.6) and hence $f_{1} \in \pi_{*}\left(\hat{E}_{\lambda}(\hat{H})\right)$ by (7.7). But this means that $f$ satisfies (7.2) with $h$ replaced by $f_{1}$, and (7.3) tells us that $f \in \pi_{*}\left(\hat{E}_{\lambda}(\hat{H})\right)$, i.e. (7.7).

Proposition 7.1 does not exclude the case that $\pi_{*} \circ \hat{E}_{\lambda_{i}}=0$ for some eigenvalue $\lambda_{i}$ of $\hat{P}$, such that $\lambda_{i}$ is not an eigenvalue of $P$. It just says that under suitable assumptions each eigenvalue of $P$ is also an eigenvalue for $\hat{P}$. It seems quite difficult indeed, to show the injectivity of $\pi_{* \mid \hat{E}_{\lambda}(\hat{H})}$ for general $\lambda$, even for particular examples. $^{27}$ (But see also the last paragraph of Example 7.6.) For $\lambda \in \Gamma$, however, we have

7.3. Lemma. Suppose Theorem 6.1(c) applies to $\hat{P}$ together with a Borel measure $\hat{m}$ satisfying for all $C, D \in \mathscr{D}$

$$
\hat{m}\left\{\hat{x} \in \hat{C} \cap \pi^{-1} D: V_{k}(\pi \hat{x}) \nsubseteq D\right\} \rightarrow 0 \quad \text { as } k \rightarrow \infty .
$$

Then $\hat{E}_{\lambda} \hat{f}=0$ for all $\lambda \in \Gamma$ and all $\hat{f} \in L_{\hat{m}}^{1}$ with $\pi_{*} \hat{f}=0 \hat{m} \circ \pi^{-1}$-a.e. (For $V_{k}(x)$ see Definition 3.3.) In particular $\pi_{*}: \operatorname{range}\left(\hat{E}_{\lambda}\right) \rightarrow L_{\hat{m} \circ \pi^{-1}}^{\infty}$ is 1-1.

Proof. Let $N_{0}=\left\{x \in X: \pi_{*} \hat{f}(x) \neq 0\right\}$. Fix $\delta>0$ and choose a decomposition $\mathscr{D}=\mathscr{D}_{1} \cup \mathscr{D}_{2}$ with a finite $\mathscr{D}_{1}$ and such that $\left\|\hat{f}_{2}\right\|_{\mathscr{A}}<\delta$ where $\hat{f}_{2}:=\sum_{D \in \mathscr{D}_{2}} \hat{f}$. $\chi_{\hat{D}}, \hat{f}_{1}:=\hat{f}-\hat{f}_{2}$. For $k \in \mathbb{N}$ let

$$
B_{k}=\left\{x \in X: \exists D \in \mathscr{D}_{1} \text { such that } x \in D \text { but } V_{k}(x) \nsubseteq D\right\} \text {. }
$$

Then

$$
\begin{aligned}
\pi_{*}\left|\hat{E}_{\lambda} \hat{f}\right|(x) \leq & \left\|\hat{E}_{\lambda} \hat{f}_{2}\right\|_{\mathscr{A}}+\mid\left\|\hat{E}_{\lambda}\left(\hat{f}_{1} \cdot\left(\chi_{N_{0} \cup B_{k}} \circ \pi\right)\right)\right\|_{\mathscr{A}} \\
& +\pi_{*}\left|\hat{E}_{\lambda}\left(\hat{f}_{1} \cdot\left(\chi_{X \backslash\left(N_{0} \cup B_{k}\right)} \circ \pi\right)\right)\right|(x) .
\end{aligned}
$$

Now

$$
\left\|\hat{E}_{\lambda} \hat{f}_{2}\right\|_{\mathscr{A}} \leq \delta\left\|\hat{E}_{\lambda}\right\|_{\mathscr{A}}
$$

and

$$
\begin{aligned}
& \left\|\hat{E}_{\lambda}\left(\hat{f}_{1} \cdot\left(\chi_{N_{0} \cup B_{k}} \circ \pi\right)\right)\right\|_{\mathscr{A}} \leq \text { const } \cdot\left\|\hat{E}_{\lambda}\right\|_{1} \int_{\pi^{-1}\left(N_{0} \cup B_{k}\right)}\left|\hat{f}_{1}\right| d \hat{m} \\
& \quad \leq \text { const } \cdot\left\|\hat{E}_{\lambda}\right\|_{1}\|\hat{f}\|_{\mathscr{A}}\left(\hat{m}\left(\pi^{-1} N_{0}\right)+\sum_{C \in \mathscr{D}_{1}} \hat{m}\left(\hat{C} \cap \pi^{-1} B_{k}\right)\right)
\end{aligned}
$$

where the constant is the norm of $\operatorname{Id}:\left(\operatorname{range}\left(\hat{E}_{\lambda}\right),\|\cdot\|_{1}\right) \rightarrow\left(\operatorname{range}\left(\hat{E}_{\lambda}\right),\|\cdot\|_{\mathscr{A}}\right)$. Since for $j \geq k$ and $y \in X \backslash B_{k}$ there is just one $\hat{x} \in \hat{X}$ such that $\hat{T}^{j} \hat{y}=\hat{x}$

\footnotetext{
27 Added in proof. Recently V. Baladi and the author proved the injectivity of $\pi_{* \mid \hat{E}_{\dot{i}}(\hat{H})}$ for $|\lambda|>\hat{r}_{\text {ess }}$ in the case of piecewise monotonic transformations. The proof can be adapted to quite general systems. It will appear in Comm. Math. Phys. as Lemma 4.3 of the paper Zeta functions and transfer operators for piecewise monotone transformations.
} 
for all $\hat{y} \in \pi^{-1}\{y\}$ (see Lemma 3.10(c)), we have

$$
\begin{aligned}
\pi_{*}\left|\left(\lambda^{-1} \hat{P}\right)^{j}\left(\hat{f}_{1} \cdot\left(\chi_{X \backslash\left(N_{0} \cup B_{k}\right)} \circ \pi\right)\right)\right|(x) \\
\quad \leq \sum_{y \in T^{-\jmath} x}\left|g_{n}(y)\right| \cdot \chi_{X \backslash\left(N_{0} \cup B_{k}\right)}(y) \cdot\left|\pi_{*} \hat{f}_{1}(y)\right| .
\end{aligned}
$$

For $y \in X \backslash N_{0}$ we have $\left|\pi_{*} \hat{f}_{1}(y)\right|=\left|\pi_{*} \hat{f}_{2}(y)\right| \leq\left|\left\|\hat{f}_{2} \mid\right\|_{\mathscr{A}}<\delta\right.$, such that the third term in (7.8) can be estimated by

$$
\begin{gathered}
\lim _{n \rightarrow \infty} \frac{1}{n} \sum_{j=k}^{n-1} \pi_{*}\left|\left(\lambda^{-1} \hat{P}\right)^{j}\left(\hat{f}_{1} \cdot\left(\chi_{X \backslash\left(N_{0} \cup B_{k}\right)} \circ \pi\right)\right)\right|(x) \\
\leq \delta \cdot \lim _{n \rightarrow \infty} \frac{1}{n} \sum_{j=k}^{n-1} P_{g}^{n} 1 \leq \delta \cdot\left\|\hat{E} e_{*} 1\right\|_{\mathscr{A}}
\end{gathered}
$$

where $\hat{E}$ is the projection onto $\left\{\hat{P}_{\hat{g}} \hat{\phi}=\hat{\phi}\right\}$.

Putting everything together we have in the limit $k \rightarrow \infty: \pi_{*}\left|\hat{E}_{\lambda} \hat{f}\right| \leq \delta C_{1}$ with a constant $C_{1}$ independent of $\delta$, and as $\delta>0$ was arbitrary, $\pi_{*}\left|\hat{E}_{\lambda} \hat{f}\right|=0$, i.e. $\hat{E}_{\lambda} \hat{f}=0$.

7.4. Remark. Theorem 6.1 (d) underlines the importance of measures $\hat{m}$ on $\hat{X}$ with

$$
\operatorname{supp}(\hat{m})=\hat{X} \quad \text { and } \quad \int \hat{P} \hat{f} d \hat{m}=\int \hat{f} d \hat{m} \quad \text { for } \hat{f} \in L_{\hat{m}}^{1}
$$

Similarly there may be a measure $m$ on $X$ with

$$
\operatorname{supp}(m)=X \quad \text { and } \quad \int P f d m=\int f d m \quad \text { for } f \in L_{m}^{1} .
$$

The relation between such measures on $X$ and $\hat{X}$ is as follows: If $m$ satisfies (7.10), then $\hat{m}:=m \circ \pi_{*}$ satisfies (7.9), observe only Lemma 4.6. On the other hand suppose that some $\hat{m}$ sastisfies (7.9) and that Lemma 7.3 applies to $\hat{P}$ and $\hat{m}$. We show that $m:=\hat{m} \circ e_{*}$ satisfies (7.10):

$$
\int P f d m-\int f d m=\int\left(e_{*} P-\hat{P} e_{*}\right) f d \hat{m}=\int \hat{E}_{1}\left(e_{*} P-\hat{P} e_{*}\right) f d \hat{m}=0
$$

by Lemma 7.3 , since $\pi_{*}\left(e_{*} P-\hat{P} e_{*}\right)=0$ by (4.13) and Lemma 4.6.

Observe that in any case $\int \hat{f} d \hat{m}=0$ if $\pi_{*} \hat{f} \equiv 0$.

\section{B. Examples.}

7.5. Example I (topological Markov shift).

Suppose that Theorem 6.1(b) applies to the system (cf. Example 6.12). Although $P$ and $\hat{P}$ coincide as operators on $\operatorname{mb}(X)=\operatorname{mb}(\hat{X})$, we distinguish between $P: C(X) \rightarrow C(X)$ and $\hat{P}: \mathscr{H}^{\infty}(\mathscr{A}) \rightarrow \mathscr{H}^{\infty}(\mathscr{A})$, and Proposition 7.1 (a) still tells us that if $|\lambda|=r(P)$ and $\left\|P^{n}\right\|=O\left(r(P)^{n}\right)$, then each continuous eigenfunction of $P$ with eigenvalue $\lambda$ belongs to $\mathscr{H}^{\infty}(\mathscr{A})$. For finite 
state Markov shifts part (b) of that proposition implies that if $E_{\lambda} f=f$ and if $f$ varies by at most $C \cdot \gamma_{2}^{n}$ on cylinders of length $n$ (where $\gamma_{2}<\frac{|\lambda|}{r(P)}$ ), then $f \in \mathscr{H}^{\infty}(\mathscr{A})$. As $\hat{r}_{\text {ess }}=0$, it suffices to choose $\beta=1, \gamma_{1}>r(\hat{P})$, and $\alpha$ close to 0 and to use Lemma 6.5 in order to show that if $\hat{f}_{n}$ is constant on cylinders of length $n$, then $\left\|\hat{P}^{n} \hat{f}_{n}\right\|_{\mathscr{A}} \leq$ const $\cdot\left\|\hat{f}_{n}\right\|_{\infty} \cdot\left\|\hat{P}^{n} 1\right\|_{\mathscr{A}}$.

7.6. Example II (up-and-down-counter, cf. 3.13 and 4.9).

Although the same remarks as above apply, this does not really help in this case, because eigenfunctions may be (and will be in general) discontinuous and cannot be approximated as before. ( $\mathscr{D}$ does not consist of cylinders only!) Hence Proposition 7.1 must be applied in a different way.

In Example 3.13 we saw that $\mathscr{I}=\left\{F_{i}, G_{i}: i \geq 0\right\}$ is the only irreducible component of $(\mathscr{D}, \rightarrow)$ not consisting of a single loop only, and Theorem 6.11.c) provides a $\sigma$-finite measure $\hat{m}$ on $\hat{X}$ such that (7.9) holds for $\hat{P}_{\lambda_{1}^{-1}}$. Since $D \backslash \operatorname{int}(D) \subseteq\left\{0^{\infty}, 1^{\infty}\right\}$ for all $D \in \mathscr{D}$, and since for cylinders $\hat{Z} \in \hat{\mathcal{Z}}_{n}$

$$
\hat{m}(\hat{Z})=\lambda_{1}^{-n} \int \hat{P}^{n} \chi_{\hat{Z}} d \hat{m} \leq \lambda_{1}^{-n} \sup _{D \in \mathscr{D}} m(D),
$$

we can apply Lemma 7.3. (Observe that $\lambda_{1}>1$, cf. Example 6.14). Hence Remark 7.4 shows that the measure $m=\hat{m} \circ e_{*}$ on $X$ satisfies (7.10) for the operator $P_{\lambda_{1}^{-1}}$. In particular $m(Z)=\hat{m}\left(e_{*} \chi_{Z}\right) \leq$ const $\cdot \lambda_{1}^{-n}$ for $Z \in \mathscr{Z}_{n}$ by (7.11).

Hence, if $f \in \operatorname{mb}(X)$ is of bounded variation with respect to the lexicographic ordering on $X$, and if $f_{n}=\sum_{Z \in \mathscr{Z}_{n}} \chi_{Z} \cdot \frac{1}{m(Z)} \int_{Z} f d m$, then

$$
\int_{X}\left|f-f_{n}\right| d m \leq m(X) \cdot \lambda_{1}^{-n} \cdot \operatorname{var}(f)
$$

and $\hat{P}^{n}\left(e_{*} f_{n}\right) \in \mathscr{H}^{\infty}(\mathscr{A})$. Since also

$$
\left\|\hat{P}^{n}\left(e_{*} f_{n}\right)\right\|_{\mathscr{A}} \leq \text { const } \cdot\|f\|_{\infty} \cdot\left\|\hat{P}^{n}\left(e_{*} 1\right)\right\|_{\mathscr{A}} \leq\|f\|_{\infty} \cdot \text { const } \cdot \lambda_{1}^{n}
$$

by Lemma 6.5, we can apply Proposition 7.1(b) with $\hat{H}=\mathscr{C}^{\infty}(\mathscr{A}), H=$ $L_{m}^{1}, \gamma_{1}=\lambda_{1}=r(P), \hat{r}_{\text {ess }}=1, \gamma_{2}=\lambda_{1}^{-1}$, and $\beta=1$, which results in the following conditions:

$$
|\lambda|>\lambda_{1}^{\frac{1}{1+1+}}, \quad|\lambda|>\lambda_{1}^{\frac{a}{1++1}} \text {. }
$$

The optimal choice for $\alpha$ is 1 in which case we obtain $|\lambda|>\sqrt{\lambda_{1}}$.

We resume: If $f \in L_{m}^{1}$ is of bounded variation and if $(P-\lambda \text { Id })^{k} f=0$ for some $k \geq 0$ and $|\lambda|>\sqrt{\lambda_{1}}$, then $f=\pi_{*} \hat{f}$ for some $\hat{f} \in \hat{E}_{\lambda}\left(\mathscr{C}^{\infty}(\mathscr{A})\right)$. Also $\lambda_{1}$ is a simple eigenvalue for $P: L_{m}^{1} \rightarrow L_{m}^{1}$ in view of Lemma 7.3, and if $M \nsubseteq k \cdot \mathbf{N}$ for all $k \geq 2$, then the irreducible part of $(\mathscr{D}, \rightarrow)$ has period 2 , and $r(P)$ and $-r(P)$ are the only eigenvalues of maximal modulus for $P$. Unfortunately this result tells nothing about those $\lambda$ with $1=\hat{r}_{\text {ess }}<|\lambda| \leq \sqrt{\lambda_{1}}$. Better results cannot be expected, however, unless one can show directly that 
$f \in \pi_{*}\left(\mathscr{H}^{\infty}(\mathscr{A})\right)$, as it has been done for certain interval maps in Lemma 11 of [Hofbauer/Keller, 1984].

7.7. Example III (piecewise monotonic transformations).

Suppose that $\tilde{\mathcal{Z}}$ is finite. In just the same way as in the foregoing example, but using the Lebesgue measure, which is a priori given, instead of the measure $m$ constructed there, one can check (A6) when $H=L_{m}^{1}, \hat{H}=\mathscr{H}^{\infty}(\mathscr{A}), \gamma_{1}=$ $\lambda_{1}=r(P)=1, \hat{r}_{\text {ess }} \leq g_{\infty}, \gamma_{2}=g_{\infty}+\varepsilon$ (any $\left.\varepsilon>0\right), \beta=1$, and $\alpha=1$. The resulting condition on $\lambda$ is $|\lambda|>\sqrt{g_{\infty}}$. Again there is a gap to $G_{\mathscr{F}}=\{|z|<$ $\left.g_{\infty}^{-1}\right\}$ (cf. 6.14).

Proposition 7.1(a) and Lemma 7.3 show that for $\lambda=1$ there is a 1-1correspondence between eigenfunctions of $\hat{P}: \mathscr{H}^{\infty}(\mathscr{A}) \rightarrow \mathscr{H}^{\infty}(\mathscr{A})$ and of $P: L_{m}^{1} \rightarrow L_{m}^{1}$.

\section{INVARIANT MEASURES}

\section{A. Preliminaries.}

Suppose that $(X, T, \mathscr{Z}, g)$ is a weighted, piecewise compact, $\mathscr{A}$-expanding system with a canonical Markov extension $(\hat{X}, \hat{T}, \hat{Z}, \hat{g})$ whose Markov diagram is uniformly forward finite. Let $P=P_{g}, \hat{P}=\hat{P}_{\hat{g}}$, and assume that there is a Borel probability measure $m$ on $X$ with $\int P f d m=\int f d m$ for all $f \in L_{m}^{1}$. Let $\hat{m}=m \circ \pi_{*}$ be the "lift" of $m$ to $\hat{X}$ (see Remark 7.4). If $\hat{r}_{\text {as }}<r(\hat{P})$ for some projection net $\mathscr{F}$, then Theorem 6.1 applies to $\hat{P}$. In particular $\lambda_{1}=1$, and for each $0 \leq \hat{h} \in L_{\hat{m}}^{1}$ such that $\hat{P} \hat{h}=\hat{h}$ and $\int \hat{h} d \hat{m}=1$ the probability measure $d \hat{\mu}=\hat{h} d \hat{m}$ is $\hat{T}$-invariant. It is easily seen that $d \mu:=h d m$ is $T$-invariant, where $h=\pi_{*}(\hat{h})$, and that

$$
\int f d \mu=\int f \circ \pi d \hat{\mu} \text { for all } f \in L_{\mu}^{1} .
$$

8.1. Remark. $T$ acts isometrically on $L_{\mu}^{2}$, and its adjoint $T^{*}$ satisfies $T^{*} f=$ $P(f h) / h . \quad T^{*} T=\operatorname{Id}_{L_{\mu}^{2}}$, whereas $T T^{*}$ is the orthogonal projection onto $T\left(L_{\mu}^{2}\right)$ (see Lemma 9 in [Hofbauer/Keller, 1982]). For $f \in L_{\mu}^{2}$ and $\lambda \in \mathbb{C}$ with $|\lambda|=1$ we have $T f=\lambda f$ if and only if $T^{*} \bar{f}=\lambda \bar{f}$ if and only if $P(\bar{f} h)=\lambda \bar{f} h$ (ibid., Theorem 2).

8.2. Lemma. $(T, \mu)$ is weakly mixing if and only if

$$
\int f d \mu=0 \Rightarrow \pi_{*} \hat{E}_{i}(f \circ \pi \cdot \hat{h}) \equiv 0 \quad\left(f \in L_{\mu}^{1}, \lambda \in \Gamma\right)
$$

Proof. Assume (8.2), let $f_{1} \in L_{\mu}^{\infty}, f_{2} \in L_{\mu}^{1}$, and let $\int f_{2} d \mu=0$. Then $\pi_{*}\left(f_{2} \circ \pi \cdot \hat{h}\right)=f_{2} h$, and

$$
\int f_{1} \circ T^{n} \cdot f_{2} d \mu=\int f_{1} \cdot \pi_{*} \hat{P}^{n}\left(f_{2} \circ \pi \cdot \hat{h}\right) d m \rightarrow 0 \quad \text { as } n \rightarrow \infty
$$

by Theorem $6.1(\mathrm{c})$, i.e. $(T, \mu)$ is weakly mixing. 
Conversely, suppose that $(T, \mu)$ is weakly mixing. Fix $k \in \mathbb{N}$ such that $\lambda_{i}^{k}=1$ for all $\lambda_{i} \in \Gamma$. Let $f_{1} \in L_{\mu}^{\infty}, f_{2} \in L_{\mu}^{1}$, and suppose that $\int f_{2} d \mu=0$. Then $f_{2} \cdot h=\pi_{*}\left(f_{2} \circ \pi \cdot \hat{h}\right)$ and

$$
\begin{aligned}
& \int f_{1} \circ T^{k n} \cdot f_{2} d \mu=\int f_{1} \cdot P^{k n}\left(f_{2} h\right) d m \\
& \quad=\int f_{1} \cdot \pi_{*} \hat{P}^{k n}\left(f_{2} \circ \pi \cdot \hat{h}\right) d m \rightarrow \sum_{\lambda \in \Gamma} \int f_{1} \cdot \pi_{*} \hat{E}_{\lambda}\left(f_{2} \circ \pi \cdot \hat{h}\right) d m
\end{aligned}
$$

as $n \rightarrow \infty$. Since $(T, \mu)$ is weakly mixing, this implies $\sum_{\lambda \in \Gamma} \pi_{*} \hat{E}_{\lambda}\left(f_{2} \circ \pi \cdot \hat{h}\right) \equiv$ 0 . Now use (4.13), Lemma 4.6, and (6.2) for $\hat{P}$ to show that $\pi_{*} \hat{E}_{\mu} e_{*} \pi_{*} \hat{E}_{\lambda}=$ $\delta_{\mu, \lambda} \pi_{*} \hat{E}_{\mu}$ for $\lambda, \mu \in \Gamma$. Then (8.2) follows at once.

\section{B. Equilibrium states.}

We recall some concepts from ergodic theory specialized to piecewise invertible systems. General references are e.g. [Parry, 1969], [Walters, 1981], or [Petersen, 1983].

Suppose $(X, T, \mathscr{Z})$ is a generating, piecewise invertible system and $\mathscr{B}$ is the Borel- $\sigma$-algebra of $X$. By $\mathscr{M}(T)$ denote the set of $T$-invariant Borel probability measures on $X$. For $\nu \in \mathscr{M}(T)$ let

$$
g_{\nu}=\sum_{Z \in \mathscr{Z}} \chi_{Z} \cdot \mathbf{E}_{\nu}\left[\chi_{Z} \mid T^{-1} \mathscr{B}\right]
$$

and

$$
h(\nu, T)=-\int \log g_{\nu} d \nu,
$$

the entropy of $(T, \nu)$. (Note that $\nu\left\{g_{\nu}=0\right\}=0$.) From the general theory of entropy it follows that $h(T, \nu)$ does not depend on the particular choice of $\mathscr{Z}$ as long as $\mathscr{Z}$ generates.

Given a weight function $g \geq 0$ and $\nu \in \mathscr{M}(T)$, let

$$
F(\nu, T, g)=h(\nu, T)+\int \log g d \nu .
$$

If $\nu\{g=0\}>0$, then $F(\nu, T, g)=-\infty$.

$\nu_{0} \in \mathscr{M}(T)$ is an equilibrium state for $\log g$, if

$$
F\left(\nu_{0}, T, g\right)=\sup \{F(\nu, T, g): \nu \in \mathscr{M}(T)\} .
$$

If $(X, d)$ is a compact metric space and if $T$ and $\log g$ are continuous, then the common value of both sides of $(8.6)$ coincides with the pressure

where

$$
\operatorname{press}(T, \log g)=\lim _{\varepsilon \rightarrow 0} \varlimsup_{n \rightarrow \infty} \frac{1}{n} \log p_{n}(T, \log g, \varepsilon),
$$

$$
p_{n}(T, \log g, \varepsilon)=\sup \left\{\sum_{x \in E} g_{n}(x): E \text { is }(n, \varepsilon) \text {-separated }\right\} \text {. }
$$

(Recall that $E \subseteq X$ is $(n, \varepsilon)$-separated, if whenever $x, y \in E$ and $x \neq y$, there exists some $i$ with $0 \leq i \leq n-1$ and $d\left(T^{i} x, T^{i} y\right)>\varepsilon$.) This variational principle is due to [Walters, 1976]. 
8.3. Theorem. Let $(X, T, \mathscr{Z}, g)$ be as in part $\mathrm{A}$ of this section with the measures $m$ and $\mu$ described there.

(a) $\mu$ is an equilibrium state for $\log g$ and $F(\mu, T, g)=0 \quad\left(=\log \lambda_{1}\right)$.

(b) Suppose $\operatorname{supp}(m)=X$. Then each equilibrium state $\nu$ for $\log g$ can be represented as $d \nu=h_{\nu} d m$ with $h_{\nu} \in \pi_{*}\left(\operatorname{range}\left(\hat{E}_{1}\right)\right)$, and the number of ergodic equilibrium states for $\log g$ equals $\operatorname{rank}\left(\pi_{*} \circ \hat{E}_{1}\right)$. In particular, if $\lambda_{1}=1$ is a simple eigenvalue of $\hat{P}_{\hat{g}}$, then there is a unique equilibrium state for $\log g$.

Proof. (a) (a slight generalisation of [Ledrappier, 1974]). For $\nu \in \mathscr{M}(T)$ let $P_{\nu}=P_{g_{\nu}}$, and observe that since we can choose $\mathbf{E}_{\nu}\left[\chi_{Z} \mid T^{-1} \mathscr{B}\right]=T T_{\nu}^{*} \chi_{Z}$,

$$
\int f_{1} \cdot P_{\nu} f_{2} d \nu=\int f_{1} \circ T \cdot f_{2} d \nu
$$

if both sides of this equation are well defined. In particular, since $\nu$ is $T$ invariant, $P_{\nu} 1=1 \nu$-a.e. Let $\alpha_{n}$ be a measurable function such that $\log \alpha_{n} \in$ $L_{\nu}^{1}$. Then

$$
\begin{aligned}
F(\nu, T, g)=h(\nu, T)+\int \log g d \nu=\int \log \frac{g}{g_{\nu}} d \nu \\
=\frac{1}{n}\left(-\int \log \alpha_{n} d \nu+\int \log \left[\alpha_{n} \circ T^{n} \prod_{k=0}^{n-1} \frac{g \circ T^{k}}{g_{\nu} \circ T^{k}}\right] d \nu\right) \quad \text { as } \nu \circ T^{-1}=\nu \\
\leq \frac{1}{n}\left(-\int \log \alpha_{n} d \nu+\int \alpha_{n} \circ T^{n} \prod_{k=0}^{n-1} \frac{g \circ T^{k}}{g_{\nu} \circ T^{k}} d \nu-1\right) \\
=\frac{1}{n}\left(-\int \log \alpha_{n} d \nu+\int P_{\nu}^{n}\left(\alpha_{n} \circ T^{n} \prod_{k=0}^{n-1} \frac{g \circ T^{k}}{g_{\nu} \circ T^{k}}\right) d \nu-1\right) \quad \text { by }(8.7) \\
\leq \frac{1}{n}\left(-\int \log \alpha_{n} d \nu+\int P^{n}\left(\alpha_{n} \circ T^{n}\right) d \nu-1\right) \\
=\frac{1}{n}\left(-\int \log \alpha_{n} d \nu+\int \alpha_{n} \cdot \pi_{*} \hat{P}^{n}\left(e_{*} 1\right) d \nu-1\right)
\end{aligned}
$$

Specialising to $\alpha_{n}=\exp (-\sqrt{n})$ we obtain

$$
F(\nu, T, g) \leq \frac{1}{\sqrt{n}}+\frac{\exp (-\sqrt{n})}{n}\left\|\hat{P}^{n}\right\|_{\mathscr{A}} \rightarrow 0 \quad \text { as } n \rightarrow \infty
$$

because $\left\|\hat{P}^{n} \mid\right\|=O\left(n^{\sigma-1} \lambda_{1}^{n}\right)=O\left(n^{\sigma-1}\right)$, where $\sigma$ is the order of the pole $\lambda_{1}$ of the resolvent of $\hat{P}$.

On the other hand, using the convention $0 \cdot \log 0=0$, we have for $h=$ $\pi_{*}(\hat{h}): h|\log h| \leq \max \left\{e^{-1},\|\hat{h}\|_{, \mathscr{\gamma}} \cdot \log \mid\|\hat{h}\|_{, \mathcal{J}}\right\}<\infty$. Hence $h \cdot \log h \in L_{m}^{1}$, i.e. $\log h \in L_{\mu}^{1}$, and as $\mu \circ T^{-1}=\mu$,

$$
\log \frac{h \circ T}{h} \in L_{\mu}^{1} \quad \text { and } \quad \int \log \frac{h \circ T}{h} d \mu=0 .
$$


Now

$$
\begin{aligned}
-F(\mu, T, g) & =\int \log \frac{h \circ T \cdot g_{\mu}}{h \cdot g} d \mu \\
& \leq \int \frac{h \circ T \cdot g_{\mu}}{h \cdot g} d \mu-1 \leq \int \frac{h \circ T \cdot g_{\mu}}{g} d m-1 \\
& =\int P\left(\frac{h \circ T \cdot g_{\mu}}{g}\right) d m-1=\int P_{\mu}(1) \cdot h d m-1 \\
& =0 \text { as } P_{\mu}(1)=1 \mu \text {-a.e. }
\end{aligned}
$$

i.e. $\mu$ maximizes $F(\nu, T, g)$. This proves part (a).

We turn to (b): Since $\operatorname{supp}(m)=X$, we have $\operatorname{supp}(\hat{m})=\hat{X}$, and hence $\Gamma$ is a finite group and all $\lambda \in \Gamma$ are semisimple (Theorem 6.1). In particular there is $k>0$ such that $\lambda^{k}=1$ and $\sum_{i=0}^{k-1} \lambda^{i}=0$ for all $1 \neq \lambda \in \Gamma$. Using the notation $\hat{\Psi}=\hat{P}-\sum_{\lambda \in \Gamma} \lambda \hat{E}_{\lambda}$ we can fix $\beta>1$ and $0<\gamma<1$ such that $\beta r(\hat{\Psi})^{k}<\gamma<1$. Let $h=\pi_{*} \hat{E}_{1}\left(e_{*} 1\right), \alpha_{n}=\min \left\{h^{-1}, \beta^{[n / k]}\right\}$, and let $\nu$ be any equilibrium state for $\log g$. Then $F(\nu, T, g)=0$, and by (8.8) we have for large $j$

$$
\begin{aligned}
0 & =k \cdot F(\nu, T, g) \\
& \leq \frac{1}{j k}\left(k \int \log \frac{1}{\alpha_{j k}} d \nu+k \int \alpha_{j k} \pi_{*} \hat{E}_{1}\left(e_{*} 1\right) d \nu-k+\sum_{i=j k}^{j k+k-1} \int \alpha_{j k} \pi_{*} \hat{\Psi}^{i}\left(e_{*} 1\right) d \nu\right) \\
& \leq \frac{1}{j}\left(\int_{\left\{h>\beta^{-\jmath}\right\}} \log h d \nu-j \log \beta \cdot \nu\left\{h \leq \beta^{-j}\right\}+\int \alpha_{j k} h d \nu-1+\gamma^{j}\right) \\
& \leq \frac{1}{j} \int_{\left\{h>\beta^{-\jmath}\right\}} \log h d \nu-\log \beta \cdot \nu\left\{h \leq \beta^{-j}\right\}+\frac{\gamma^{j}}{j} .
\end{aligned}
$$

Hence

$$
\log \beta \cdot \nu\left\{h \leq \beta^{-j}\right\} \leq \frac{1}{j} \int_{\left\{h>\beta^{-j}\right\}} \log h d \nu+\frac{\gamma^{j}}{j}
$$

As $\log h$ is bounded from above, $-\infty \leq \int \log h d \nu<\infty$ is well defined. This shows $\nu\{h=0\}=0$ (let $j \rightarrow \infty$ in (8.9)).

We prove $\log h \in L_{\nu}^{1}$ : Suppose for a contradiction that $\int \log h d \nu=-\infty$. Then $\int_{\left\{h>\beta^{-j}\right\}} \log h d \nu \leq 0$ for large $j$ and (8.9) yields $\nu\left\{h \leq \beta^{-j}\right\} \leq \gamma^{j}$ for large $j$, which in turn implies $\int \log h d \nu>-\infty$. This allows the following 
estimate:

$$
\begin{aligned}
1 & =\int \frac{h}{h} d \nu=\int \frac{P h}{h} d \nu=\int P\left(\frac{h}{h \circ T}\right) d \nu \\
& =\int P_{\nu}\left(\frac{h \cdot g}{h \circ T \cdot g_{\nu}}\right) d \nu=\int \frac{h \cdot g}{h \circ T \cdot g_{\nu}} d \nu \\
& \geq 1+\int \log \left(\frac{h \cdot g}{h \circ T \cdot g_{\nu}}\right) d \nu=1+\int \log \left(\frac{g}{g_{\nu}}\right) d \nu \\
& =1+F(\nu, T, g)=1
\end{aligned}
$$

with equality if and only if $(h \cdot g) /\left(h \circ T \cdot g_{\nu}\right)=1 \quad \nu$-a.e. Hence $g_{\nu}=$ $(g \cdot h) /(h \circ T) \quad \nu$-a.e. Therefore we obtain for $f \in L_{\nu}^{1}$ and $n \in \mathbb{N}$

$$
\begin{aligned}
\int f d \nu & =\int P_{\nu}^{n} f d \nu=\int \frac{P^{n}(f h)}{h} d \nu \\
& =\int \frac{\pi_{* *} \hat{P}^{n}(f \circ \pi \cdot \hat{h})}{h} d \nu .
\end{aligned}
$$

In view of Theorem $6.1(\mathrm{~d})$ there are $\hat{T}-\hat{T}^{-1}$-invariant sets $\hat{X}_{i}$ and $\hat{P}$-invariant functions $\hat{h}_{i} \geq 0$ such that $\int_{\hat{X}_{i}} \hat{h}_{j} d \hat{m}=\delta_{i, j}$ and

$$
\hat{E}_{1} \hat{f}=\sum_{i=1}^{d} \int_{\hat{X}_{i}} \hat{f} d \hat{m} \cdot \hat{h}_{i} \quad \text { for } \hat{f} \in L_{\hat{m}}^{1} .
$$

In particular $\hat{h}=\sum_{j=1}^{d} \alpha_{j} \hat{h}_{j}$ for some $\alpha_{j} \in \mathbb{R}$.

Suppose now $f$ is bounded and constant on each cylinder $Z \in \mathscr{Z}_{N}$ for some $N>0$. Then $\hat{P}^{N}(f \circ \pi \cdot \hat{h}) \in \mathscr{H}^{\infty}(\mathscr{A})$ and $\frac{1}{n} \sum_{k=N}^{n+N-1} \hat{P}^{k}(f \circ \pi \cdot \hat{h}) \rightarrow$ $\hat{E}_{1} \hat{P}^{N}(f \circ \pi \cdot \hat{h})$ in $\mathscr{H}^{\infty}(\mathscr{A})$. Hence, for such $f,(8.10)$ implies

$$
\begin{aligned}
\int f d \nu & =\int \frac{\pi_{*} \hat{E}_{1} \hat{P}^{N}(f \circ \pi \cdot \hat{h})}{h} d \nu \\
& =\sum_{i, j=1}^{d} \alpha_{j} \cdot \int_{\hat{X}_{i}} \hat{P}^{N}\left(f \circ \pi \cdot \hat{h}_{j}\right) d \hat{m} \cdot \int \frac{\pi_{*} \hat{h}_{i}}{h} d \nu \\
& =\sum_{i=1}^{d} \alpha_{i} \int f \circ \pi \cdot \hat{h}_{i} d \hat{m} \cdot \int \frac{\pi_{*} \hat{h}_{i}}{h} d \nu \\
& =\int f \cdot\left(\sum_{i=1}^{d} \alpha_{i} \int \frac{\pi_{*} \hat{h}_{i}}{h} d \nu \cdot \pi_{*} \hat{h}_{i}\right) d m \\
& =\int f h_{\nu} d m
\end{aligned}
$$

for some $h_{\nu} \in \pi_{*}\left(\operatorname{range}\left(\hat{E}_{1}\right)\right)$. Since $(X, T, \mathscr{Z})$ generates the Borel- $\sigma$-algebra of $X$, it follows that $d \nu=h_{\nu} d m$.

8.4. Remark. If $m\left\{x \in D: V_{k}(x) \nsubseteq D\right\} \rightarrow 0$ as $k \rightarrow \infty$ for all $D \in \mathscr{D}$, then Lemma 7.3 applies, and there are exactly $\operatorname{rank}\left(\hat{E}_{1}\right)$ ergodic equilibrium states for $\log g$ in Theorem 8.3(b). 


\section{Exponential weak Bernoulli mixing.}

Let $(X, T, \mathscr{Z}, g), m$, and $\mu$ be as described in part A of this section. For finite or countable partitions $\mathscr{R}$ and $\mathscr{S}$ of $X$ let

$$
D_{\mu}(\mathscr{R}, \mathscr{S})=\sum_{R \in \mathscr{R}, S \in \mathscr{S}}|\mu(R \cap S)-\mu(R) \mu(S)| .
$$

$\mathscr{Z}$ is called a weak Bernoulli partition for $(T, \mu)$, if

$$
\lim _{n \rightarrow \infty} b(n)=0 \quad \text { where } b(n)=\sup _{k, l \in \mathbb{N}} D_{\mu}\left(\mathscr{Z}_{k}, T^{-(k+n)} \mathscr{Z}_{l}\right) .
$$

It is well known that

$$
b(n)=2 \cdot \sup _{k \in N} \int \sup \left\{\left|\mu\left(A \mid \mathscr{Z}_{k}\right)-\mu(A)\right|: A \in T^{-(n+k)} \mathscr{B}_{\mathscr{X}}\right\} d \mu
$$

where $\mathscr{B}_{\mathscr{X}}$ is the $\sigma$-algebra generated by $\bigvee_{i=0}^{\infty} T^{-i} \mathscr{Z} . \quad \frac{1}{2} b(n)$ is known as the mixing coefficient of absolute regularity of the $\mathscr{Z}$-valued stochastic process $\xi_{n}, \xi_{n}(x)=Z$ if $T^{n}(x) \in Z$. A discussion of this notion of mixing and a lot of references are provided by [Bradley, 1983].

8.5. Theorem. Suppose that $(T, \mu)$ is weakly mixing. Then $\mathscr{Z}$ is a weak Bernoulli partition for $(T, \mu)$ and

$$
b(n)=O\left(r^{n}\right)
$$

for each $r>\max \left\{|\lambda| / \lambda_{1}: \lambda \in \sigma(\hat{P}),|\lambda|<\lambda_{1}\right\}$.

8.6. Remark. For finite state Markov chains an even stronger mixing property was proved by [Bowen, 1975]. For piecewise monotonic interval transformations (with $g$ of bounded variation) a result like Theorem 8.5 can be found in [Hofbauer/Keller, 1982] and [Rychlik, 1983]. Here we adapt Rychlik's very elegant proof to our setting.

Proof of the theorem. Let $A=T^{-(n+k)} \check{A}, \check{A} \in \mathscr{B}_{\mathscr{X}}$. On $B \in \mathscr{Z}_{k}$ we have

$$
\mu\left(A \mid \mathscr{Z}_{k}\right)=\frac{1}{\mu(B)} \int_{A} \chi_{B} h d m=\frac{1}{\mu(B)} \int_{\dot{A}} P^{n+k}\left(\chi_{B} h\right) d m .
$$

Since $\mu(A)=\mu(\check{A})=\int_{\dot{A}} h d m$,

and hence

$$
\left|\mu\left(A \mid \mathscr{X}_{k}\right)-\mu(A)\right| \leq \int_{\dot{A}}\left|P^{n+k}\left(\chi_{B} h\right) / \mu(B)-h\right| d m \text { on } B
$$

$$
\frac{1}{2} b(n) \leq \sup _{k \in N_{B \in \mathscr{Z}_{k}}} \int\left|P^{n+k}\left(\chi_{B} h\right)-\mu(B) \cdot h\right| d m .
$$

Let $\hat{B}=\pi^{-1}(B)$. Then $\chi_{\hat{B}}=\chi_{B} \circ \pi, \pi_{*}\left(\chi_{\hat{B}} \hat{h}\right)=\chi_{B} h$, and $P^{n+k}\left(\chi_{B} h\right)=$ $\pi_{*} \hat{P}^{n+k}\left(\chi_{\hat{B}} \hat{h}\right)$. Hence

$$
\begin{aligned}
\frac{1}{2} b(n) & \leq \sup _{k \in N_{B}} \sum_{B \in Z_{k}} \int\left|\pi_{*} \hat{P}^{n+k}\left(\left(\chi_{\hat{B}}-\mu(B)\right) \hat{h}\right)\right| d m \\
& =\sup _{k \in N_{B}} \sum_{B \in Z_{k}} \int\left|\pi_{*} \hat{\Psi}^{n} \hat{P}^{k}\left(\left(\chi_{\hat{B}}-\mu(B)\right) \hat{h}\right)\right| d m
\end{aligned}
$$


by Lemma 8.2 , where $\hat{\Psi}=\hat{P}-\sum_{\lambda \in \Gamma} \lambda \hat{E}_{\lambda}$ as the $L_{\hat{m}}^{1}$-operator (cf. Theorem $6.1(\mathrm{c})$. Now

$$
\begin{aligned}
\frac{1}{2} b(n) & \leq \sup _{k \in \mathbb{N}} \sum_{B \in \mathscr{Z}_{k}}\left\|\hat{\Psi}^{n} \hat{P}^{k}\left(\left(\chi_{\hat{B}}-\mu(B)\right) \hat{h}\right)\right\| \|_{\mathscr{A}} \\
& \leq \text { const } \cdot r^{n} \cdot \sup _{k \in \mathbb{N}} \sum_{B \in \mathscr{Z}_{k}}\left(\left\|\hat{P}^{k}\left(\chi_{\hat{B}} \cdot \hat{h}\right)\right\|\left\|_{\mathscr{A}}+\mu(B) \cdot\right\| \hat{h}\|\|_{\mathscr{A}}\right) \\
& \leq \text { const } \cdot r^{n}\left(\|\hat{h}\|_{\mathscr{A}}+\sup _{k \in \mathbb{N}} \sum_{\substack{\omega \text { path in } \mathscr{Z} \\
\text { oflength } k}}\left\|\left(\hat{h} \cdot \hat{g}_{n}\right) \circ T_{\omega}^{-1}\right\|_{\mathscr{A}}\right) \\
& \leq \text { const } \cdot r^{n}\left(\|\hat{h}\|_{\mathscr{A}}+\sup _{k \in \mathbb{N}}\left\|\hat{P}^{k}\left(\hat{h}^{*}\right)\right\|_{\mathscr{A}}\right)
\end{aligned}
$$

by Lemma 6.5 where

$$
\hat{h}^{*}=\sum_{\hat{Z} \in \hat{Z}} \chi_{\hat{Z}} \cdot\left\|\hat{h}_{\mid \hat{Z}}\right\|_{\mathscr{A}} .
$$

Finally observe that $\sup _{k \in \mathbb{N}}\left\|\hat{P}^{k}\right\|_{\mathscr{A}}<\infty$ and $\hat{h}^{*} \in \mathscr{H}^{\infty}(\mathscr{A})$.

\subsection{Example II (up-and-down-counter).}

In (7.6) we saw that the counter $\left(\Omega\left(W_{M}\right), S\right)$ has a Borel measure $m$ with $\operatorname{supp}(m)=\Omega\left(W_{M}\right)$ and $\int P f d m=\lambda_{1} \int f d m$ for all $f \in L_{m}^{1} \cdot \lambda_{1}$ is a simple eigenvalue of $\hat{P}$. Replacing $g=1$ by $g=\lambda_{1}^{-1}$, we can apply Theorem 8.3 and see that the counter has a unique measure $\mu$ of maximal entropy $\log \lambda_{1}$ for each subset $M$ of $\mathbb{N}$. If $M \nsubseteq k \cdot \mathbb{N}$ for all $k \geq 2$, then 1 and -1 are the only peripheral eigenvalues of $\hat{P}_{\lambda_{1}^{-1}}$. Hence $\left(\Omega\left(W_{M}\right), S^{2}\right)$ has exactly two ergodic measures of maximal entropy, both weakly mixing, and hence exponentially weak Bernoulli mixing under $S^{2}$.

\subsection{Example III (piecewise monotonic transformations).}

Suppose that $\mathscr{Z}$ is finite. In Example 6.14 we showed, how Theorem 6.1 applies. In particular, if $\tilde{g}=1 /\left|\tilde{T}^{\prime}\right|$, then all equilibrium states on $X$ for $\log g$ are absolutely continuous with respect to the Lebesgue measure on $X$. The number of ergodic equilibrium states for $\log g$ equals $\operatorname{rank}\left(\hat{E}_{1}\right)$, and in view of Example 7.7 this number is identical with the multiplicity of the eigenvalue 1 for the operator $P$ acting on the space of functions of bounded variation on $X$ or on $L_{m}^{1}(X)$ (cf. [Hofbauer/Keller, 1982]).

As the spaces $[0,1]=\tilde{X}$ and $X$ differ only by countably many points, " $X$ " may be replaced by " $[0,1]$ " in the above remarks. 


\section{ApPlications}

\section{A. Limiting behaviour of processes $S_{n}=\sum_{k=0}^{n-1} f \circ T^{k}$.}

Let $(X, T, \mathscr{Z}, g)$ be as in $\S 8$.A, and assume that $(T, \mu)$ is weakly mixing. Fix $f \in L_{\mu}^{2}$ with $\int f d \mu=0$ and suppose

$$
\sum_{k=1}^{\infty}\left\|\left(T^{*}\right)^{k} f\right\|_{L_{\mu}^{2}}<\infty
$$

$\left(Y_{k}\right)_{k \geq 0}=\left(f \circ T^{k}\right)_{k \geq 0}$ is a strictly stationary stochastic process with respect to the probability measure $\mu$ on $X$. Let $S_{n}=\sum_{k=0}^{n-1} Y_{k}$ and

$$
\sigma^{2}=\operatorname{var}\left(Y_{0}\right)+2 \cdot \sum_{k=1}^{\infty} \operatorname{cov}\left(Y_{0}, Y_{k}\right)<\infty
$$

An easy classical calculation [Kac, 1946] yields

$$
\lim _{n \rightarrow \infty} \operatorname{var}\left(S_{n} / \sqrt{n}\right)=\sigma^{2}
$$

The exceptional case $\sigma^{2}=0$ is characterized by the following lemma (see Lemma 6 of [Rousseau-Egele, 1983]; we give a shorter proof).

9.1. Lemma. Assume (9.1). Then $\sigma^{2}=0$ if and only if $f=\phi-T \phi$ for some $\phi \in L_{\mu}^{2}$.

Proof. By (9.1), $f_{\infty}=\sum_{k=0}^{\infty}\left(T^{*}\right)^{k} f$ is in $L_{\mu}^{2}$. A straightforward calculation yields

$$
\begin{aligned}
\sigma^{2} & =\int\left(f_{\infty}+T^{*} f_{\infty}\right)\left(f_{\infty}-T^{*} f_{\infty}\right) d \mu \\
& =\left\|f_{\infty}\right\|_{L_{\mu}^{2}}^{2}-\left\|T^{*} f_{\infty}\right\|_{L_{\mu}^{2}}^{2} \\
& =\left\|f_{\infty}\right\|_{L_{\mu}^{2}}^{2}-\left\|T T^{*} f_{\infty}\right\|_{L_{\mu}^{2}}^{2} .
\end{aligned}
$$

Since $T T^{*}$ is the orthogonal projection onto $T L_{\mu}^{2}, \sigma^{2}=0$ is equivalent to $f_{\infty}=T T^{*} f_{\infty}$, which is true if $f=\phi-T \phi$ and which, on the other hand, implies $f=f_{\infty}-T^{*} f_{\infty}=T T^{*} f_{\infty}-T^{*} f_{\infty}$.

Let $f_{n}=\mathbf{E}_{\mu}\left[f \mid \mathscr{Z}_{n}\right]$. A convenient tool to verify (9.1) is

9.2. Lemma. Suppose $f \in L_{\mu}^{2}$ is bounded, $\int f d \mu=0$, and

$$
\left\|f_{n}-f\right\|_{L_{\mu}^{2}}=O\left(a^{n}\right)
$$

for some $0<a<1$. Then there is $0<b<1$ such that $\left\|\left(T^{*}\right)^{n} f\right\|_{L_{\mu}^{2}}=O\left(b^{n}\right)$. 
Proof. Fix $k \in \mathbb{N}$ such that $\lambda^{k}=1$ for all $\lambda \in \Gamma$, and suppose that $n=2 l k$ for some $l \in \mathbb{N}$. Let $\hat{\Psi}=\hat{P}-\sum_{\lambda \in \Gamma} \lambda \hat{E}_{\lambda}$ (as $L_{m}^{1}$-operator). Then

$$
\begin{aligned}
\left\|\left(T^{*}\right)^{n} f_{l k}\right\|_{L_{i}^{2}}^{2} & \leq\left\|f_{l k}\right\|_{\infty} \cdot \int\left|P^{n}\left(f_{l k} \cdot h\right)\right| d m \\
& \leq\|f\|_{\infty} \cdot \int\left|\pi_{*} \hat{P}^{n}\left(f_{l k} \circ \pi \cdot \hat{h}\right)\right| d m \\
& \leq\|f\|_{\infty} \cdot\left(\sum_{\lambda \in \Gamma} \int\left|\pi_{*} \hat{E}_{\lambda}\left(f_{l k} \circ \pi \cdot \hat{h}\right)\right| d m+\int\left|\pi_{*} \hat{\Psi}^{n}\left(f_{l k} \circ \pi \cdot \hat{h}\right)\right| d m\right) \\
& =\|f\|_{\infty} \cdot \int\left|\pi_{*} \hat{\Psi}^{l k} \hat{P}^{l k}\left(f_{l k} \circ \pi \cdot \hat{h}\right)\right| d m
\end{aligned}
$$

by Lemma 8.2 and as $\hat{\Psi} \hat{P}=\hat{\Psi}^{2}$,

$$
\begin{aligned}
& \leq\|f\|_{\infty} \cdot\left\|\hat{\Psi}^{l k} \hat{P}^{l k}\left(f_{l k} \circ \pi \cdot \hat{h}\right)\right\|_{\mathscr{A}} \\
& \leq\|f\|_{\infty} \cdot\left\|\hat{\Psi}^{l k} \mid\right\|_{\mathscr{A}} \cdot\|f\|_{\infty} \cdot\left\|\hat{P}^{l k} \hat{h}^{*}\right\|_{\mathscr{A}}
\end{aligned}
$$

by Lemma 6.5 , where $\hat{h}^{*}=\sum_{Z \in \hat{\mathcal{X}}} \chi_{Z}\left\|\hat{h}_{\mid Z}\right\|_{\mathscr{A}}$,

$$
=O\left(\left\|\hat{\Psi}^{n / 2}\right\|_{\mathscr{A}}\right) \quad \text { as } \sup _{n}\left\|\hat{P}^{n}\right\|_{\mathscr{A}}<\infty
$$

Since the spectral radius of $\hat{\Psi}$ is less than 1 , the lemma follows from $\left\|f_{l k}-f\right\|_{L_{\mu}^{2}}=O\left(a^{n / 2}\right)$ and the fact that $\left\|T^{*}\right\|_{L_{\mu}^{2}}=1$.

There are (at least) four approaches to probabilistic limit theorems for the process $S_{n}$ provided $\sigma^{2}>0$.

9.3. $L^{2}$-TECHNIQUE.

Assuming (9.4) and using Lemma 9.2 one can reduce the asymptotic normality of $S_{n} / \sqrt{n \sigma^{2}}$ to that of a backward martingale (see [Gordin, 1969]). This approach to the central limit theorem was used by [Keller, 1980] for piecewise monotonic interval maps.

\section{4. $\left(f \circ T^{n}\right)_{n \geq 0}$ AS A FUNCTIONAL OF AN ABSOLUTELY REGULAR PROCESS.}

Let $\xi_{n}(x)=Z \in \mathscr{Z}$ if $T^{n} x \in Z$. This defines a stationary $\mathscr{Z}$-valued process, which, by Theorem 8.5 , is absolutely regular with exponential decrease of mixing coefficients. Assuming (9.4) and observing that $(X, T, \mathscr{Z})$ generates, the process $\left(Y_{n}\right)_{n \geq 0}$ can be represented as a sufficiently regular functional of the process $\left(\xi_{n}\right)_{n \geq 0}$, and one obtains a central limit theorem ${ }^{28}$ with convergence rate $n^{-\nu}$ for some $0<\nu<\frac{1}{2}$ and an almost sure invariance principle ${ }^{29}$ for the process $S_{n}$ that is good enough to imply loglog-laws and weak invariance principles. Details can be found in [Hofbauer/Keller, 1982] where this approach has been used for piecewise monotonic interval maps.

\footnotetext{
${ }^{28}$ Cf. [Ibragimov/Linnik, 1971].

${ }^{29}$ Cf. [Philipp/Stout, 1975].
} 
The other techniques rely on direct estimates of the Fourier- or Laplacetransforms of $S_{n} / \sqrt{n}$ or $S_{n}$. They apply only to "analytic" $f$, analytic in the sense that for each $Z \in \mathscr{Z}$ there is $\tilde{f}_{Z} \in \mathscr{H}^{\infty}\left(U_{Z}\right)$ such that $\tilde{f}_{Z} \circ s=f_{\mid Z}$ (cf. $\S 5)$.

\subsection{Fourier-TRANSFORM TECHNIQUe.}

Let $P_{t}=P_{g \cdot \exp (i t f)}$ for $t \in \mathbb{R}$. Then Theorem 6.1 applies to $P_{t}$,

$$
\int \exp \left(i t S_{n}\right) d \mu=\int P^{n}\left(h \cdot \exp \left(i t S_{n}\right)\right) d m=\int P_{t}^{n}(h) d m,
$$

and a perturbation expansion of the spectral representation of $\hat{P}_{t}$ at $t=0$ leads to a convergence rate $n^{-1 / 2}$ in the central limit theorem, while this expansion for small $|t|$ together with the spectral theory of $\hat{P}_{t}$ for arbitrary $t$ leads to a local limit theorem for $S_{n}$. [Rousseau-Egele, 1983] has carried out this program for piecewise monotonic interval maps.

\subsection{LAPLACE-TRANSFORM TECHNIQUE.}

We still assume $f$ to be analytic in the above sense, but we skip the assumption $\int f d \mu=0$. Let now $P_{t}=P_{\exp (t f)}$ for $t \in \mathbf{R}$. Then, similarly as above, $\int \exp \left(t S_{n}\right) d m=\int P_{t}^{n}(1) d m$, and Theorem 6.1(b) (or perturbation theory applied to $\left.\hat{P}_{0}\right)$ yields quasicompacity of $\hat{P}_{t}$ for small $|t|$, say $\hat{P}_{t}=\lambda_{1}(t) \hat{E}_{1}(t)+\hat{\Psi}(t)$ for $t_{-}<t<t_{+}$and $\hat{E}_{1}(t)(\hat{f})=\hat{h} \cdot \int \hat{f} d \hat{\mu}$. (Remember that $(T, \mu)$ is weakly mixing.)

Let $\psi_{n}(t)=\frac{1}{n} \log \int \exp \left(t S_{n}\right) d m$. Then

(a) $\psi_{n}(t)=\frac{1}{n} \log \int \pi_{*} \hat{P}_{t}^{n}\left(e_{*} 1\right) d m \rightarrow \log \lambda_{1}(t)=: \psi(t)<\infty \quad(n \rightarrow \infty)$,

(b) $\psi(t)$ is real analytic on $\left(t_{-}, t_{+}\right)$, since $\hat{P}_{t}$ is an analytic family of operators,

(c) using Chapter VII, $\S 2.3$ and formula $(2.14)$ in [Kato, 1976] it is not hard to show that $\psi^{\prime}(0)=\int f d \mu$ and $\psi^{\prime \prime}(0)=\sigma^{2}$.

If $\sigma^{2}>0$ one can choose $t_{-}, t_{+}$so small that $\psi^{\prime \prime}(t)>0$ for $t_{-}<t<t_{+}$. Let $\alpha_{-}=\psi^{\prime}\left(t_{-}\right), \alpha_{+}=\psi^{\prime}\left(t_{+}\right)$. Properties (a)-(c) are sufficient for a large deviations result, namely:

For all $\alpha \in\left(\psi^{\prime}(0), \alpha_{+}\right)$

$$
\lim _{n \rightarrow \infty} \frac{1}{n} \log m\left(\frac{S_{n}}{n}>\alpha\right)=-\mathrm{I}(\alpha) \in(-\infty, 0),
$$

and for all $\alpha \in\left(\alpha_{-}, \psi^{\prime}(0)\right)$

$$
\lim _{n \rightarrow \infty} \frac{1}{n} \log m\left(\frac{S_{n}}{n}<\alpha\right)=-\mathrm{I}(\alpha) \in(-\infty, 0),
$$

where $\mathrm{I}(\alpha)=\alpha \cdot t_{\alpha}-\psi\left(t_{\alpha}\right)$ and $t_{\alpha}$ is the unique solution of $\psi^{\prime}(t)=\alpha$. (Note that if $\int f d \mu=0$, then $\mathrm{I}(\alpha)=\alpha^{2} / 2 \sigma^{2}+O\left(\alpha^{3}\right)$ in the limit $\alpha \rightarrow 0$.)

Here we used [Plachky/Steinebach, 1975]. A good discussion of the Laplacetransform technique can be found in [Cox/Griffeaths, 1984]. 
B. Limiting behaviour of statistics based on $\left(f \circ T^{n}\right)_{n \geq 0}$.

The technique of representing $\left(f \circ T^{n}\right)_{n \geq 0}$ as a functional of an exponentially mixing absolutely regular process (cf. 9.4) can be used to derive limit theorems for statistics based on the sample $\left(f(x), \ldots, f \circ T^{n-1}(x)\right)$, more complicated than the simple "mean-value statistic" $S_{n}$. This has been worked out for $U$ statistics by [Denker/Keller, 1985]. We do not go into details.

\section{Extinction probabilities and transient behaviour.}

Suppose $(X, T, \mathscr{Z}, g)$ is a weighted system and $m$ a probability measure on $X$ such that $\int P f d m=\int f d m$ for all $f \in L_{m}^{1}$. Let $A$ be a finite union of cylinders from $\mathscr{Z}$ and let

$$
\tau(x)=\tau_{A}(x)=\min \left\{n \geq 0: T^{n} x \in A\right\} .
$$

(For other "traps" one must start with a refined partition $\mathscr{Z}$.) We investigate $p_{n}=m\{\tau \geq n\}$. Since $\tau \geq n$ is equivalent to $\frac{1}{n} \sum_{k=0}^{n-1} \chi_{A} \circ T^{k} \leq 0$, the large deviation result from (9.6) (if applicable to $f=\chi_{A}$ ) would yield $\lim _{n \rightarrow \infty} \frac{1}{n} \log p_{n}=-\mathrm{I}(0)$. But as $f=\chi_{A} \geq 0, \lambda_{1}(t)$ is increasing in $t$ and hence $\psi^{\prime}(t) \geq 0$ such that $\alpha_{-} \geq 0$, i.e. $\alpha=0$ is not in the domain of validity of the large deviation estimate. There is an alternative approach to this problem, however, which shares the large deviation flavour:

Let $g_{A}=g \cdot\left(1-\chi_{A}\right)$. (Do not confuse this notation with the former $g_{n}$.) Suppose that Theorem 6.1 applies to $\left(\hat{X}, \hat{T}, \hat{\mathcal{Z}}, \hat{g}_{A}\right)$ (it need not apply to $(\hat{X}, \hat{T}, \hat{Z}, \hat{g}) !)$. Then

$$
\begin{aligned}
p_{n} & =\int \prod_{k=0}^{n-1}\left(1-\chi_{A}\right) \circ T^{k} d m=\int P_{g}^{n}\left(\prod_{k=0}^{n-1}\left(1-\chi_{A}\right) \circ T^{k}\right) d m \\
& =\int P_{g_{A}}^{n}(1) d m=\int \pi_{*} \hat{P}_{\hat{g}_{A}}^{n}\left(e_{*} 1\right) d m,
\end{aligned}
$$

i.e. $\varlimsup_{n \rightarrow \infty} \frac{1}{n} p_{n} \leq \log r\left(\hat{P}_{\hat{g}_{A}}\right)$.

Similarly, if $\lambda_{1, A}=r\left(\hat{P}_{\hat{g}_{A}}\right)$ and $\hat{P}_{\hat{g}_{A}} \hat{h}_{A}=\lambda_{1, A} \hat{h}_{A} \geq 0$, then

$$
\begin{aligned}
p_{n} & \geq\left\|\hat{h}_{A}\right\|_{\mathscr{A}}^{-1} \cdot \int \prod_{k=0}^{n-1}\left(1-\chi_{A}\right) \circ T^{k} \cdot \pi_{*}\left(\hat{h}_{A}\right) d m \\
& =\left\|\hat{h}_{A}\right\|_{\mathscr{A}}^{-1} \cdot \int \pi_{*} \hat{P}_{\hat{g}_{A}}^{n}\left(\hat{h}_{A}\right) d m \\
& =\left\|\hat{h}_{A}\right\|_{\mathscr{A}}^{-1} \cdot \lambda_{1, A}^{n} \cdot \int \pi_{*}\left(\hat{h}_{A}\right) d m
\end{aligned}
$$

and hence $\lim _{n \rightarrow \infty} \frac{1}{n} \log p_{n}=\log \lambda_{1, A}$ provided $\int \pi_{*}\left(\hat{h}_{A}\right) d m>0$, e.g. if $\operatorname{supp}(m)=X$. 
Theorem 8.3 provides an equilibrium state $\mu_{A} \in \mathscr{M}(T)$ with $F\left(\mu_{A}, T, g_{A}\right)=$ $\log \lambda_{1, A}$. In particular, $\mu_{A}\left\{g_{A} \circ T^{k}=0\right\}=0$ for all $k \geq 0$, i.e. $\operatorname{supp}\left(\mu_{A}\right)=$ : $X_{A} \subseteq \bigcap_{k=0}^{\infty} T^{-k}(X \backslash A), g_{\mid X_{A}}>0$, and

$$
\begin{aligned}
\log \lambda_{1, A} & =F\left(\mu_{A}, T_{\mid X_{A}}, g_{\mid X_{A}}\right) \\
& =\sup \left\{F\left(\nu, T_{\mid X_{A}}, g_{\mid X_{A}}\right): \nu \in \mathscr{M}\left(T_{\mid X_{A}}\right)\right\} \\
& =\operatorname{press}\left(T_{\mid X_{A}}, \log g_{\mid X_{A}}\right)
\end{aligned}
$$

provided $X_{A}$ is a compact metric space (cf. §8.B).

We summarize these results in

9.7. Proposition. If $X \backslash A$ is compact metric and if $\operatorname{supp}(m)=X$, then

$$
\lim _{n \rightarrow \infty} \frac{1}{n} \log m\left\{\tau_{A} \geq n\right\}=\operatorname{press}\left(T_{\mid X_{A}}, \log g_{\mid X_{A}}\right) .
$$

For constant $g$ this was proved in [Keller, 1984].

If the trap $A$ is very small, it may take quite a long time until a trajectory hits it with probability $\frac{1}{2}$, say, and it is interesting to characterize the behaviour of the orbits before extinction. We shall show that it is in some sense indistinguishable from the behaviour of typical orbits of the system $\left(T, \mu_{A}\right)$ where $\mu_{A}$ is an equilibrium state for $\log g_{A}$.

So suppose again that $(X, T, \mathscr{Z}, g)$ is a weighted system and $m$ is a probability measure on $X$ with $\operatorname{supp}(m)=X$ and $\int P f d m=\int f d m$ for all $f \in L_{m}^{1} . m$ is lifted to a measure $\hat{m}=m \circ \pi_{*}$ on $\hat{X}$. Let the trap $A$, the first exit time $\tau_{A}$, and the weight function $g_{A}$ be as above.

9.8. Proposition. Suppose that Lemma 6.9 applies to the system $\left(\hat{X}, \hat{T}, \hat{\mathcal{Z}}, \hat{g}_{A}\right)$, that $\lambda_{1, A}$ is the only peripheral eigenvalue of $\hat{P}_{\hat{g}_{A}}$ and that $\lambda_{1, A}$ is semisimple, i.e. $\hat{P}_{\hat{g}_{A}}=\lambda_{1, A} \hat{E}_{1}+\hat{\Psi}$ with $r(\hat{\Psi})<\lambda_{1, A}$. Let $\hat{m}_{A}=\left(\hat{m} \circ \hat{E}_{1}\left(e_{*} 1\right)\right)^{-1} \cdot \hat{m} \circ \hat{E}_{1}$, $m_{A}=\hat{m}_{A} \circ e_{*}$, and for $n \in \mathbb{N}$ let $m_{n}=m\left(\cdot \mid \tau_{A} \geq n\right)$.

For $\Phi: X \rightarrow \mathbb{R}$ and $n \in \mathbb{N}$ let $\operatorname{Var}_{n}(\Phi)=\sup \{|\Phi(x)-\Phi(y)|: x, y \in Z \in$ $\left.\mathscr{Z}_{n}\right\}$. Then there are $S>0$ and $0<q<1$ such that

$$
\left|\int \Phi d m_{n}-\int \Phi d m_{A}\right| \leq S \cdot\|\Phi\|_{\infty} \cdot q^{n-k}+\operatorname{Var}_{k}(\Phi)
$$

for all $0<k<n$ and $\Phi: X \rightarrow \mathbb{R}$.

Proof. For $0<k<n$ let

$$
\begin{gathered}
m_{k, n}=\sum_{Z \in \mathscr{X}_{k}} m_{A}(\boldsymbol{Z}) \cdot m_{n}(\cdot \mid Z), \\
\nu_{k, n}=\sum_{Z \in \mathscr{Z}_{k}} m_{A}(\boldsymbol{Z}) \cdot m_{n}(\cdot \mid \boldsymbol{Z}) \times m_{A}(\cdot \mid \boldsymbol{Z}) .
\end{gathered}
$$


$m_{k, n}$ is a probability measure on $X, \nu_{k, n}$ on $X \times X$, and

$$
\begin{aligned}
& \left|\int_{X} \Phi d m_{n}-\int_{X} \Phi d m_{A}\right| \\
& \leq\left|\int_{X} \Phi d m_{n}-\int_{X} \Phi d m_{k, n}\right|+\left|\int_{X \times X} \Phi \circ \operatorname{pr}_{1} d \nu_{k, n}-\int_{X \times X} \Phi \circ \operatorname{pr}_{2} d \nu_{k, n}\right| \\
& \quad \text { where } \operatorname{pr}_{1}, \mathrm{pr}_{2}: X \times X \rightarrow X \text { are canonical projections } \\
& \quad \leq \sum_{Z \in \mathscr{Z}_{k}}\left|m_{n}(Z)-m_{A}(Z)\right|\left|\int_{Z} \Phi d m_{n}(\cdot \mid Z)\right|+\operatorname{Var}_{k}(\Phi) \\
& \leq 2\|\Phi\|_{\infty} \cdot \sup _{B}\left|m_{n}(B)-m_{A}(B)\right|+\operatorname{Var}_{k}(\Phi)
\end{aligned}
$$$$
\text { where } \operatorname{pr}_{1}, \operatorname{pr}_{2}: X \times X \rightarrow X \text { are canonical projections, }
$$

where the supremum extends over all $B$ in the $\sigma$-algebra $\sigma\left(\mathscr{Z}_{k}\right)$ generated by $\mathscr{Z}_{k}$.

Next, if $p_{n}=m\{\tau \geq n\}>0$, then

$$
\begin{aligned}
m_{n}(B) & =p_{n}^{-1} \int_{\{\tau \geq n\}} \chi_{B} d m \\
& =p_{n}^{-1} \int P^{n}\left(\chi_{B} \cdot \prod_{i=0}^{n-1}\left(1-\chi_{A}\right) \circ T^{i}\right) d m \\
& =p_{n}^{-1} \int \pi_{*} \hat{P}_{\hat{g}_{A}}^{n-k} \hat{f} d m \quad \text { where } \hat{f}=\hat{P}_{\hat{g}_{A}}^{k}\left(e_{*} \chi_{B}\right) \in \mathscr{H}^{\infty}(\mathscr{A}) \\
& =\frac{\lambda_{1, A}^{n-k} \int \hat{E}_{1} \hat{f} d \hat{m}+\int \hat{\Psi}^{n-k} \hat{f} d \hat{m}}{\lambda_{1, A}^{n} \int \hat{E}_{1}\left(e_{*} 1\right) d \hat{m}+\int \hat{\Psi}^{n}\left(e_{*} 1\right) d \hat{m}} \\
& =\frac{\lambda_{1, A}^{n} \int \hat{E}_{1}\left(e_{*} \chi_{B}\right) d \hat{m}+\int \hat{\Psi}^{n-k} \hat{f} d \hat{m}}{\lambda_{1, A}^{n} \int \hat{E}_{1}\left(e_{*} 1\right) d \hat{m}+\int \hat{\Psi}^{n}\left(e_{*} 1\right) d \hat{m}}
\end{aligned}
$$

Here we made use of the fact that $\hat{m} \circ \hat{E}_{1}$ is a $\sigma$-finite measure on $\hat{X}$ invariant under $\lambda_{1, A}^{-1} \hat{P}_{\hat{g}_{A}}$ (see Lemma 6.9).

Fix $0<\tilde{q}<q<1$ such that $\lambda_{1}^{-1} r(\hat{\Psi})<\tilde{q}$. As

$$
\begin{aligned}
\left\|\hat{\Psi}^{n-k} \hat{f}\right\|_{\mathscr{A}} & \leq \text { const } \cdot\left(\lambda_{1, A} \tilde{q}\right)^{n-k} \cdot\|\hat{f}\|_{\mathscr{A}} \leq \text { const } \cdot\left(\lambda_{1, A} \tilde{q}\right)^{n-k}\left(\lambda_{1, A} q / \tilde{q}\right)^{k} \\
& \leq \text { const } \cdot \lambda_{1, A}^{n} q^{n-k} \quad \text { (use Lemma 6.5) }
\end{aligned}
$$

and

$$
\left\|\mid \hat{\Psi}^{n}\left(e_{*} 1\right)\right\| \|_{\mathscr{A}} \leq \mathrm{const} \cdot\left(\lambda_{1, A} q\right)^{n}
$$

we finally get

$$
\begin{aligned}
m_{n}(B) & =\int e_{*} \chi_{B} d \hat{m}_{A} \cdot\left(1+O\left(q^{n}\right)\right)+O\left(q^{n-k}\right) \\
& =m_{A}(B)+O\left(q^{n-k}\right)
\end{aligned}
$$

uniformly in $B$. 
9.9. Corollary. Let $F_{n}(n \in \mathbb{N})$ be a sequence of functions from $X^{n}$ to $\mathbb{R}$, and assume that $F_{n}$ is Lipschitz-continuous in each variable separately with a Lipschitz-constant $L_{n}$, in the sense that (for some $\left.\delta>0\right) \mid F_{n}(\ldots, x, \ldots)-$ $F_{n}(\ldots, y, \ldots) \mid \leq L_{n} \cdot \Delta_{\delta}(x, y)$, where $\Delta_{\delta}(x, y)=\exp (-\delta \cdot \max \{n \in \mathbb{N}: \exists Z \in$ $\mathscr{Z}_{n}$ such that $\left.\left.x, y \in Z\right\}\right)$.

If $\sup _{n \in \mathrm{N}}\left\|F_{n}\right\|_{\infty}<\infty$ and if $\lim _{n \rightarrow \infty} L_{n}=0$, then

$$
\lim _{n \rightarrow \infty}\left|\int F_{n}\left(x, \ldots, T^{n-1} x\right) d m_{n}-\int F_{n}\left(x, \ldots, T^{n-1} x\right) d m_{A}\right|=0 .
$$

Proof. Let $\Phi_{n}(x)=F_{n}\left(x, \ldots, T^{n-1} x\right)$. Then

$$
\operatorname{Var}_{k}\left(\Phi_{n}\right) \leq L_{n} \cdot\left(\frac{1}{1-e^{-\delta}}+n-k\right),
$$

and choosing $k=k_{n}$ such that $n-k_{n} \rightarrow \infty$ and $L_{n} \cdot\left(n-k_{n}\right) \rightarrow 0$ as $n \rightarrow \infty$, the corollary follows from Proposition 9.8.

9.10. Remark. If $\Xi_{n}: X \rightarrow C[0,1]$ is defined as the path obtained by linear interpolation of the points $\left(\frac{i}{n-1}, n^{-1 / 2} \sum_{j=0}^{i} f \circ T^{j}(x)\right),(i=0, \ldots, n-1)$ for some Lipschitz-continuous function $f: X \rightarrow \mathbb{R}$ (in the above-defined sense), the corollary shows that the distributions of the $\Xi_{n}$ under $m_{n}$ and under $m_{A}$ are asymptotically equivalent. The distribution of $\Xi_{n}$ under $m_{A}$ tends, in turn, to the distribution of a Brownian motion over $[0,1]$ as $n \rightarrow \infty$ provided $\sigma^{2}$ as defined in (9.2) is positive, cf. 9.4.

\section{REFERENCES}

F. Blanchard and G. Hansel (1986): Systèmes codés, Theor. Computer Science 44, 17-49.

R. Bowen (1975): Equilibrium states and the ergodic theory of Anosov diffeomorphisms, Lecture Notes in Math. vol. 470, Springer-Verlag, Berlin-Heidelberg-New York.

R. Bowen and O. E. Lanford (1970): Zeta functions of restrictions of the shift transformation, Proc. Sympos. Pure Math., vol. 14 Amer. Math. Soc., Providence, R.I., pp. 43-49.

R. C. Bradley (1983): Absolute regularity and functions of Markov chains, Stochastic Process. Appl. 14, 67-77.

F. E. Browder (1961): On the spectral theory of elliptic differential operators. I, Math. Ann. 142, 22130.

E. Coven and M. Paul(1975): Sofic systems, Israel J. Math. 20, 165-177.

J. T. Cox and D. Griffeath (1984): Large deviations for Poisson systems of independent random walks, Z. Wahrsch. Verw. Gebiete 66, 543-558.

M. Denker, C. Grillenberger, and K. Sigmund (1976): Ergodic theory on compact spaces, Lecture Notes in Math. vol. 527, Springer-Verlag, Berlin-Heidelberg-New York.

M. Denker and G. Keller (1986): Rigorous statistical procedures for data from dynamical systems, J. Statist. Phys. 44, 67-93.

J. Dugundji (1966): Topology, Allyn and Bacon, Boston, Mass.

N. Dunford and J. T. Schwartz (1958): Linear operators, Part I, Interscience, New York.

I. C. Gohberg and E. I. Sigal (1971): An operator generalization of the logarithmic residue theorem and the theorem of Rouché, Math. USSR-Sb. 13,603-625.

M. I. Gordin (1969): A central limit theorem for stationary processes, Soviet Math. Dokl. 10.11741176. 
A. Grothendieck(1955): Produits tensoriels topologiques et espaces nucléaires, Chapter II, Mem. Amer. Math. Soc. No. 16. (1956): La théorie de Fredholm, Bull. Soc. Math. France 84, 319-384.

F. Hofbauer (1979): On intrinsic ergodicity of piecewise monotonic transformations with positive entropy, Israel J. Math. 34, 213-237.

__, (1986): Piecewise invertible dynamical systems, Probab. Theory Related Fields 72, 359-386.

F. Hofbauer and G. Keller (1982): Ergodic properties of invariant measures for piecewise monotonic transformations, Math. Z. 180,119-140.

(1984): Zeta-functions and transfer operators for piecewise linear transformations, J. Reine Angew. Math. 352, 100-113.

I. A. Ibragimov and Y. V. Linnik (1971): Independent and stationary sequences of random variables, Wolters-Nordhoff, Groningen.

M. Kac (1946): On the distribution of sums of the type $\sum f\left(2^{k} t\right)$, Ann. of Math. 47, 33-49.

T. Kato (1976): Perturbation theory for linear operators, Springer-Verlag, Berlin-Heidelberg-New York.

G. Keller (1979): Ergodicité et mesures invariantes pour les transformations dilatantes par morceaux d'une région bornée du plan, C. R. Acad. Sci. Paris, Sér. A 289,625-627.

(1980): Un théorème de la limite centrale pour une classe de transformations monotones par morceaux, C. R. Acad. Sci. Paris Sér. A 291, 155-158.

(1984): On the rate of convergence to equilibrium in one-dimensional systems, Comm. Math. Phys. 96, 181-193.

(1987): Circular codes, loop counting, and zeta-functions, Preprint, Universität Heidelberg.

W. Krieger (1984): On sofic systems, I, Israel J. Math. 48, 305-330.

A. Lasota and J. A. Yorke (1973): On the existence of invariant measures for piecewise monotonic transformations, Trans. Amer. Math. Soc. 186, 481-488.

F. Ledrappier (1974): Principe variationnel et systèmes dynamiques symboliques, Z. Wahrsch. Verw. Gebiete 30, 185-202.

D. H. Mayer (1976): On a $\zeta$ function related to the continued fraction transformation, Bull. Soc. Math. France 104, 195-203.

(1980): The Ruelle-Araki transfer operator in classical statistical mechanics, Lecture Notes in Phys. vol. 123, Springer-Verlag, Berlin-Heidelberg-New York.

(1984): Approach to equilibrium for locally expanding maps in $\mathbf{R}^{k}$, Comm. Math. Phys. 95, $1-15$.

M. Mori (1985): On the decay of correlations for piecewise monotone mappings. I, Tokyo J. Math. 8, 389-414.

J. Neveu (1964): Bases mathématiques du calcul des probabilités, Masson, Paris.

R. D. Nussbaum (1970): The radius of the essential spectrum, Duke Math. J. 37, 473-478.

Y. Oono and Y. Takahashi (1980): Chaos, external noise, and Fredholm theory, Progr. Theor. Phys. 63, 1804-1807.

W. Parry (1969): Entropy and generators, Benjamin, New York.

K. Petersen (1983): Ergodic theory, Cambridge Univ. Press, Cambridge.

W. Philipp and W. Stout (1975) Almost sure invariance principles for partial sums of weakly dependent random variables, Mem. Amer. Math. Soc. No. 161.

D. Plachky and J. Steinebach (1975): A theorem about probabilities of large deviations with an application to queuing theory, Period. Math. Hungar. 6, 343-345.

M. Pollicott (1984): A complex Ruelle-Perron-Frobenius theorem and two counterexamples, Ergodic Theory amd Dynamical Systems 4, 135-146. (1986): Meromorphic extensions of generalized zeta functions, Invent. Math. 85, 147-164. 
H. J. Rousseau-Egele (1983): Un théorème de la limite locale pour une classe de transformations dilatantes et monotones par morceaux, Ann Probab. 11,772-788.

D. Ruelle (1976): Zeta-functions for expanding maps and Anosov-flows, Inventiones Math. 34, 231242.

M. Rychlik (1983): Bounded variation and invariant measures, Studia Math. 76, 69-80.

H. H. Schaefer (1980): On positive contractions in $L^{p}$-spaces, Trans. Amer. Math. Soc. 257, 261-268. (1974): Banach lattices and positive operators, Springer-Verlag, Berlin-Heidelberg-New York.

F. Schweiger (1973): The metrical theory of Jacobi-Perron algorithm, Lecture Notes in Math. vol. 334, Springer-Verlag, Berlin-Heidelberg-New York.

(1975): Some remarks on ergodicity and invariant measures, Michigan Math, J. 22, 181-187.

E. Seneta (1980): Non-negative matrices and Markov chains, 2nd ed., Springer-Verlag, BerlinHeidelberg-New York.

Y. Takahashi (1981): Fredholm determinant of unimodal linear maps, Sci. Papers College Gen. Ed. Univ. Tokyo 31, 61-87.

__ (1983): Shift with orbit basis and realization of one dimensional maps, Osaka J. Math. 20, 599629.

J. B. Wagoner (1988): Topological Markov chains, $C^{*}$-algebras and $K_{2}$, Adv. in Math. 71, 133-185.

P. Walters (1976): A variational principle for the pressure of continuous transformations, Amer. J. Math. 97, 937-971.

_. (1981): An introduction to ergodic theory, Springer-Verlag, Berlin-Heidelberg-New York.

M. S. Waterman (1970): Some ergodic properties of multi-dimensional f-expansions, Z. Wahrsch. Verw. Gebiete 16, 77-103.

B. Weiss (1973): Subshifts of finite type and sofic systems, Monatsh. Math. 77, 462-474.

Sonderforschungsbereich 123, Universität Heidelberg, D-6900 Heidelberg, Germany

Current address: Mathematisches Institut, Universität Erlangen, Bismarckstrasse $1 \frac{1}{2}, 8520$, Erlangen, Federal Republic of Germany 